TRANSACTIONS OF THE

AMERICAN MATHEMATICAL SOCIETY

Volume 351, Number 11, Pages 4281-4336

S 0002-9947(99)02239-4

Article electronically published on June 29, 1999

\title{
DIMENSION FORMULA FOR GRADED LIE ALGEBRAS AND ITS APPLICATIONS
}

\author{
SEOK-JIN KANG AND MYUNG-HWAN KIM
}

\begin{abstract}
In this paper, we investigate the structure of infinite dimensional Lie algebras $L=\bigoplus_{\alpha \in \Gamma} L_{\alpha}$ graded by a countable abelian semigroup $\Gamma$ satisfying a certain finiteness condition. The Euler-Poincaré principle yields the denominator identities for the $\Gamma$-graded Lie algebras, from which we derive a dimension formula for the homogeneous subspaces $L_{\alpha}(\alpha \in \Gamma)$. Our dimension formula enables us to study the structure of the $\Gamma$-graded Lie algebras in a unified way. We will discuss some interesting applications of our dimension formula to the various classes of graded Lie algebras such as free Lie algebras, Kac-Moody algebras, and generalized Kac-Moody algebras. We will also discuss the relation of graded Lie algebras and the product identities for formal power series.
\end{abstract}

\section{INTRODUCTION}

In [K1] and [Mo1], Kac and Moody independently introduced a new class of Lie algebras, called Kac-Moody algebras, as a generalization of finite dimensional simple Lie algebras over $\mathbf{C}$. These algebras are ususally infinite dimensional, and are defined by the generators and relations associated with generalized Cartan matrices, which is similar to Serre's presentation of complex semisimple Lie algebras.

In $[\mathrm{M}]$, by generalizing Weyl's denominator identity to the affine root system, Macdonald obtained a new family of combinatorial identities, including Jacobi's triple product identity as the simplest case. In [K2], Kac discovered a character formula, called the Weyl-Kac formula, for the integrable highest weight modules over symmetrizable Kac-Moody algebras. The Weyl-Kac formula, when applied to the 1-dimensional trivial representation, yields the denominator identity, and Kac showed that the Macdonald identities are just the denominator identities for affine Kac-Moody algebras. Since then, the theory of infinite dimensional Lie algebras and their representations has been the focus of extensive research activities due to its remarkable and significant applications to many areas of mathematics and mathematical physics.

On the other hand, in his study of the vertex algebras and the Moonshine conjecture [CN], Borcherds initiated the study of generalized Kac-Moody algebras [B1][B6]. The structure and the representation theories of generalized Kac-Moody algebras are similar to those of Kac-Moody algebras, and a lot of facts about KacMoody algebras can be generalized to generalized Kac-Moody algebras [B2], [K3].

Received by the editors May 23, 1997.

1991 Mathematics Subject Classification. Primary 17B01, 17B65, 17B70, 11F22.

This research was supported by NON DIRECTED RESEARCH FUND, Korea Research Foundation, 1996.

(C)1999 American Mathematical Society 
The main difference is that the generalized Kac-Moody algebras may have imaginary simple roots with norms $\leq 0$ whose multiplicity can be $>1$. The most interesting example of generalized Kac-Moody algebras may be the Monster Lie algebra, which plays a crucial role in Borcherds' proof of the Moonshine conjecture [B5].

In this paper, we consider a very general class of infinite dimensional Lie algebras which includes Kac-Moody algebras and generalized Kac-Moody algebras as special cases. More precisely, let $\Gamma$ be a countable abelian semigroup such that every element $\alpha \in \Gamma$ can be written as a sum of elements in $\Gamma$ in only finitely many ways, and consider the $\Gamma$-graded Lie algebras $L=\bigoplus_{\alpha \in \Gamma} L_{\alpha}$ with finite dimensional homogeneous subspaces. Indeed, there are many interesting and important Lie algebras that are graded by some semigroup satisfying our finiteness condition. For example, the free Lie algebras generated by graded vector spaces are of this kind, and so are the positive or negative parts of finite dimensional simple Lie algebras, Virasoro algebra, Kac-Moody algebras, and generalized Kac-Moody algebras. Here, we propose a general method for investigating the structure of these infinite dimensional Lie algebras in a unified way.

In Section 1, applying the Euler-Poincaré principle to the $\Gamma$-graded Lie algebra $L=\bigoplus_{\alpha \in \Gamma} L_{\alpha}$, we obtain a product identity of the form

$$
\prod_{\alpha \in \Gamma}\left(1-e^{\alpha}\right)^{\operatorname{dim} L_{\alpha}}=1-\sum_{\alpha \in \Gamma} d(\alpha) e^{\alpha}
$$

where $d(\alpha) \in \mathbf{Z}$ for all $\alpha \in \Gamma$. The above identity will be interpreted as the $d e$ nominator identity for the Lie algebra $L$. By taking the logarithm of both sides of the denominator identity and using Möbius inversion, we will derive a general dimension formula for the homogeneous subspaces $L_{\alpha}$ of $L$ (Theorem 1.1). Our dimension formula will be expressed in terms of the Witt partition functions associated with the partitions of the elements in $\Gamma$. In many cases, we will be able to obtain simple expressions of the Witt partition functions. Moreover, various expressions of the Witt partition functions will give rise to a number of interesting combinatorial identities.

In Section 2, we will discuss the applications of our dimension formula to the free Lie algebras $L=\bigoplus_{\alpha \in \Gamma} L_{\alpha}$ generated by graded vector spaces $V=\bigoplus_{\alpha \in \Gamma} V_{\alpha}$ with $\operatorname{dim} V_{\alpha}<\infty$ for all $\alpha \in \Gamma$. When applied to free Lie algebras, our dimension formula will be called the generalized Witt formula (Theorem 2.1; see also [Ju1], [Ka1], [Ka2], [KaK1]). If $L=\bigoplus_{n=1}^{\infty} L_{n}$ is the free Lie algebra generated by an $r$-dimensional vector space, then our formula yields the classical Witt formula (cf. [J], [S1]). We will discuss the "1-dimensional generalization" and "2-dimensional generalization" of the Witt formula, and derive a lot of interesting combinatorial identities. In particular, we obtain the exchange principle (Corollary 2.6) and the double exchange principle (Corollary 2.12). These exchange principles can be generalized to the $n$ dimensional exchange principle (Corollary 2.17).

In Section 3, we will apply our dimension formula to symmetrizable Kac-Moody algebras to derive a closed form root multiplicity formula (Theorem 3.5), which is a generalization of Berman-Moody's formula $[\mathrm{BM}]$. Our root multiplicity formula enables us to study the structure of a symmetrizable Kac-Moody algebra $\mathfrak{g}$ as a representation of a smaller Kac-Moody algebra $\mathfrak{g}_{0}$ contained in $\mathfrak{g}$. As an application, we first consider the rank 2 hyperbolic Kac-Moody algebra $\mathfrak{g}(a, b)$ associated with 
the generalized Cartan matrix $A=\left(\begin{array}{cc}2 & -a \\ -b & 2\end{array}\right)$ with $a \geq b$ and $a b>4$. The root system of the algebra $\mathfrak{g}(a, b)$ and its relation with quasi-regular cusps on Hilbert modular surfaces were investigated in [LM] (see also [Mo2]). When $a=b=3$, the real root system of the algebra $\mathfrak{g}(3,3)$ can be expressed in terms of the Fibonacci sequence $[\mathrm{F}]$. In $[\mathrm{KM} 2]$, using our root multiplicity formula, we study the structure of the algebra $\mathfrak{g}(a, a)$, and, as a by-product, we obtain a new elementary algorithm finding all the integral points on the hyperbolas $x^{2}-a x y+y^{2}=k$ for $k \leq 1$. Recently, our method was generalized to an elementary algorithm finding all the integral points on the hyperbolas $b x^{2}-c x y+a y^{2}=k$ for all $k \in \mathbf{Z}$ when $a$ and $b$ divide $c$ [HJK]. Compared with the classical method (cf. [Cas], [Hu]), this method is similar in spirit, but requires much less work. In this paper, we determine the root multiplicities of the algebra $\mathfrak{g}(a, b)$ in terms of the Fibonacci-type sequences $\left\{A_{n}\right\}_{n \geq 0}$ and $\left\{B_{n}\right\}_{n \geq 0}$ defined by (3.14) (see also [K3, Exercise 5.25]).

We will also apply our root multiplicity formula to the hyperbolic Kac-Moody algebra $\mathfrak{F}$ associated with the generalized Cartan matrix

$$
A=\left(a_{i j}\right)_{i, j=-1,0,1}=\left(\begin{array}{ccc}
2 & -1 & 0 \\
-1 & 2 & -2 \\
0 & -2 & 2
\end{array}\right) .
$$

In $[\mathrm{FF}]$, the algebra $\mathfrak{F}$ was viewed as the minimal graded Lie algebra with local part $V \oplus \mathfrak{g}_{0} \oplus V^{*}$, where $\mathfrak{g}_{0}$ is the affine Kac-Moody algebra $A_{1}^{(1)}, V$ is the basic representation of $\mathfrak{g}_{0}$, and $V^{*}$ is the contragredient module of $V$, and a very nice multiplicity formula was given for the level 2 roots. In [Ka1]-[Ka4], we generalized their construction and used homological methods to obtain a root multiplicity formula for the roots of level $\leq 5$. Here, we give a multiplicity formula for all the roots of $\mathfrak{F}$ in three different forms. First, we view the algebra $\mathfrak{F}$ as a representation of the affine Kac-Moody algebra $A_{1}^{(1)}$, and express the root multiplicities of the algebra $\mathfrak{F}$ in terms of the number of paths arising from the crystal base theory of quantum affine Lie algebras (Proposition 3.11). On the other hand, by taking $\mathfrak{F}_{0} \cong \operatorname{sl}(3, \mathbf{C})+\mathfrak{h}$, the algebra $\mathfrak{F}$ can be viewed as a representation of $\operatorname{sl}(3, \mathbf{C})$. In this case, we give a root multiplicity formula in terms of the Kostka numbers (Proposition 3.12). Finally, we can simply take $\mathfrak{F}_{0}$ to be the Cartan subalgebra $\mathfrak{h}$, in which case, we give a root multiplicity formula using the enumeration of the Weyl group $W \cong P G L(2, \mathbf{Z})$ (Proposition 3.13).

In [SUM1], N. Sthanumoorthy and A. Uma Maheswari introduced a new class of indefinite Kac-Moody algebras called the extended hyperbolic Kac-Moody algebras. For example, the Kac-Moody algebra $\mathfrak{F}(a, b)$ associated with the generalized Cartan matrix

$$
A=\left(a_{i j}\right)_{i, j=-1,0,1}=\left(\begin{array}{ccc}
2 & -a & 0 \\
-b & 2 & -2 \\
0 & -2 & 2
\end{array}\right)
$$

is an extended hyperbolic Kac-Moody algebra. In [SUM2], the algebra $\mathfrak{F}(a, b)$ was realized as the minimal graded Lie algebra with local part $V \oplus \mathfrak{g}_{0} \oplus V^{*}$, where $\mathfrak{g}_{0}$ is the affine Kac-Moody algebra $A_{1}^{(1)}, V$ is the irreducible highest weight module over $A_{1}^{(1)}$ with highest weight $\lambda$ such that $\lambda\left(h_{0}\right)=b, \lambda\left(h_{1}\right)=0$, and $V^{*}$ is the contragredient module of $V$. When $a=1$, using the homological methods developed in [Ka1] and [Ka2], N. Sthanumoorthy and A. Uma Maheswari gave a root 
multiplicity formula for the roots of level $\leq 3$. In this work, we will apply our root multiplicity formula to the algebra $\mathfrak{F}(a, b)$ and give a multiplicity formula for all the roots in terms of the number of paths (Proposition 3.14) as we did for the hyperbolic Kac-Moody algebra $\mathfrak{F}$. Our root multiplicity formula can be applied to a more general class of indefinite Kac-Moody algebras as long as they can be realized as the minimal graded Lie algebras with local part $V \oplus \mathfrak{g}_{0} \oplus V^{*}$, where $\mathfrak{g}_{0}$ is a Kac-Moody algebra of classical finite type or classical affine type, $V$ is a direct sum of irreducible highest weight modules over $\mathfrak{g}_{0}$ with dominant integral highest weights, and $V^{*}$ is the contragredient module of $V$ (cf. [BKM1]). However, the general behavior of the root multiplicities of indefinite Kac-Moody algebras is still mysterious.

In Section 4, we will apply our dimension formula to generalized Kac-Moody algebras to derive a closed form root multiplicity formula (Theorem 4.4), which enables us to study the structure of a generalized Kac-Moody algebra $\mathfrak{g}$ as a representation of a Kac-Moody algebra $\mathfrak{g}_{0}$ contained in $\mathfrak{g}$. As an application, some generalized Kac-Moody algebras will be shown to be the maximal graded Lie algebras with local part $V \oplus \mathfrak{g}_{0} \oplus V^{*}$, where $\mathfrak{g}_{0}$ is the maximal Kac-Moody subalgebra contained in $\mathfrak{g}, V$ is the direct sum of irreducible highest weight modules over $\mathfrak{g}_{0}$ with highest weight $-\alpha_{i}$ ( $\alpha_{i}$ runs over all imaginary simple roots counted with multiplicities), and $V^{*}$ is the contragredient module of $V$ (Proposition 4.5; see also [Ju1]). The choice of $\mathfrak{g}_{0}$ in our formula gives rise to various expressions of the root multiplicities of $\mathfrak{g}$, which would yield combinatorial identities. We will determine the root multiplicities of the generalized Kac-Moody algebras associated with the Borcherds-Cartan matrices of low ranks, and illustrate the above principle by producing some combinatorial identities.

In [B5], Borcherds completed the proof of the Moonshine conjecture by constructing an infinite dimensional graded Lie algebra called the Monster Lie algebra. The Monster Lie algebra is a generalized Kac-Moody algebra associated with the Borcherds-Cartan matrix $A=(-(i+j))_{i, j \in I}$ of charge $\underline{m}=(c(i) \mid i \in I)$, where $I=\{-1\} \cup\{1,2,3, \cdots\}$ is the index set for the simple roots, and the $c(n)$ are the coefficients of the elliptic modular function

$$
j(q)-744=\sum_{n=-1}^{\infty} c(n) q^{n}=q^{-1}+196884 q+21493760 q^{2}+\cdots .
$$

By applying our root multiplicity formula to the Monster Lie algebra, we obtain a recursive relation for the coefficients of the elliptic modular function $j(q)-744$ (Corollary 4.10; see also [Ka6], [Ju1], [KaK1]). In [LZ], B. Lian and G. Zuckerman gave a new construction of the Monster Lie algebra using the Moonshine cohomology functor. We expect their construction will yield many other interesting graded Lie algebras whose structures can be studied using our dimension formula.

In this work, we define the class of the Monstrous Lie algebras $L(f)$ associated with a formal power series $f(q)=\sum_{n=-1}^{\infty} f(n) q^{n}$ such that $f(-1)=1$, $f(0)=0$, and $f(n) \in \mathbf{Z}_{\geq 0}$ for all $n \geq 1$. The class of Monstrous Lie algebras contains the Monster Lie algebra as a special case. For example, the Monstrous Lie algebra associated with the elliptic modular function $j(q)-744$ is the Monster Lie algebra, and the Monstrous Lie algebras associated with the Thompson series $T_{g}(q)=\sum_{n=-1}^{\infty} c_{g}(n) q^{n}$ with $c_{g}(n) \geq 0$ for all $n \geq 1$ are the Monstrous Lie algebras considered in [B5]. We will determine the root multiplicities of the Monstrous Lie 
algebras in terms of $f(i)$ 's (Proposition 4.10). It would be interesting to investigate the relation of Monstrous Lie algebras and the (genus 0) modular functions.

Finally, in Section 5, we discuss the relation of the graded Lie algebras and the product identity for the formal power series of the form

$$
\prod_{\alpha \in \Gamma}\left(1-e^{\alpha}\right)^{A(\alpha)}=1-\sum_{\alpha \in \Gamma} d(\alpha) e^{\alpha},
$$

where $A(\alpha) \in \mathbf{Z}_{\geq 0}$ and $d(\alpha) \in \mathbf{Z}$ for all $\alpha \in \Gamma$. If $d(\alpha) \in \mathbf{Z}_{\geq 0}$ for all $\alpha \in \Gamma$, the above product identity can be interpreted as the denominator identity for the free Lie algebra $L=\bigoplus_{\alpha \in \Gamma} L_{\alpha}$ generated by the $\Gamma$-graded vector space $V=\bigoplus_{\alpha \in \Gamma} V_{\alpha}$ with $\operatorname{dim} V_{\alpha}=d(\alpha)$ for all $\alpha \in \Gamma$. Hence we have $\operatorname{dim} L_{\alpha}=A(\alpha)$, and our dimension formula will yield the combinatorial identity (5.4).

We will also discuss the applications of the above idea to some of the automorphic forms with infinite product expansions given in [B7]. In particular, the product identities for the Eisenstein series $E_{6}(\tau), E_{10}(\tau)$, and $E_{14}(\tau)$ will be interpreted as the denominator identities for some free Lie algebras, and we will obtain some interesting relations of the Fourier coefficients of the corresponding modular functions. In [Ka8], we will give a more comprehensive study of the structure of graded Lie superalgebras using the superdimension formula. For example, any product identity for a (normalized) formal power series with integral coefficients will be interpreted as the denominator identity for some free Lie superalgebra.

\section{ACKNOWLEDGEMENTS}

Part of this work was completed while the first author was visiting Yale University in the summer of 1996. He would like to express his sincere gratitude to the Department of Mathematics at Yale University for their hospitality. We would also like to thank Professors George B. Seligman, Efim Zelmanov, and Gregg Zuckerman for their interest in this work and many valuable suggestions.

\section{The DIMENSION FORMULA}

Let $\Gamma$ be a countable abelian semigroup such that every element $\alpha \in \Gamma$ can be written as a sum of elements in $\Gamma$ in only finitely many ways. For example, the semigroup $\mathbf{Z}_{>0}$ of positive integers satisfies the above condition, whereas the monoid $\mathbf{Z}_{\geq 0}$ of nonnegative integers doesn't. Another example of the semigroup satisfying our finiteness condition is $\Gamma=Q_{ \pm} \backslash\{0\}$, where $Q_{+}=\sum_{i \in I} \mathbf{Z}_{\geq 0} \alpha_{i}$ (resp. $Q_{-}=\sum_{i \in I} \mathbf{Z}_{\leq 0} \alpha_{i}$ ) is the positive (resp. negative) root lattice of finite dimensional simple Lie algebras, Kac-Moody algebras, or generalized Kac-Moody algebras.

In this paper, we consider $\Gamma$-graded Lie algebras

$$
L=\bigoplus_{\alpha \in \Gamma} L_{\alpha}, \quad \text { where } \operatorname{dim} L_{\alpha}<\infty \text { for all } \alpha \in \Gamma .
$$

There are many interesting and important Lie algebras that are graded by some semigroups satisfying our finiteness condition. For example, the free Lie algebras generated by graded vector spaces are of this kind, and so are the positive or negative parts of finite dimensional simple Lie algebras, Virasoro algebra, KacMoody algebras, and generalized Kac-Moody algebras.

An element $\alpha \in \Gamma$ is called a root of $L$ if $L_{\alpha} \neq 0$, and the homogeneous subspace $L_{\alpha}$ is called the root space attached to $\alpha$. The number mult $\alpha:=\operatorname{dim} L_{\alpha}$ is called the multiplicity of the root $\alpha$. In this work, using the Euler-Poincaré principle and 
Möbius inversion, we will derive a closed form formula for $\operatorname{dim} L_{\alpha}$ for all $\alpha \in \Gamma$. Our dimension formula enables us to study the structure of the $\Gamma$-graded Lie algebras in a unified way. We will discuss some interesting applications of our dimension formula to the various kinds of graded Lie algebras such as free Lie algebras, Kac-Moody algebras, and generalized Kac-Moody algebras. We will also discuss the relation of graded Lie algebras and the product identities for the (normalized) formal power series.

Let $\Gamma$ be an abelian semigroup. In general, for a $\Gamma$-graded vector space $V=$ $\bigoplus_{\alpha \in \Gamma} V_{\alpha}$ with $\operatorname{dim} V_{\alpha}<\infty$ for all $\alpha \in \Gamma$, we define the character of $V$ to be

$$
\operatorname{ch} V=\sum_{\alpha \in \Gamma}\left(\operatorname{dim} V_{\alpha}\right) e^{\alpha}
$$

where the $e^{\alpha}$ are the basis elements of the semigroup algebra $\mathbf{C}[\Gamma]$ with the multiplication $e^{\alpha} e^{\beta}=e^{\alpha+\beta}$ for all $\alpha, \beta \in \Gamma$. (Actually, $\operatorname{ch} V$ is usually an element of $\mathbf{C}[[\Gamma]]$, the completion of $\mathbf{C}[\Gamma]$.) Thus finding a formula for the root multiplicities of the $\Gamma$-graded Lie algebra $L=\bigoplus_{\alpha \in \Gamma} L_{\alpha}$ is equivalent to finding a formula for the character $\operatorname{ch} L=\sum_{\alpha \in \Gamma}\left(\operatorname{dim} L_{\alpha}\right) e^{\alpha}$ of $L$.

Let $\mathbf{C}$ be the trivial 1-dimensional $L$-module. Recall that the homology modules $H_{k}(L)=H_{k}(L, \mathbf{C})$ are determined from the following complex:

$$
\begin{aligned}
\cdots \longrightarrow \Lambda^{k}(L) & \stackrel{d_{k}}{\longrightarrow} \Lambda^{k-1}(L) \longrightarrow \cdots \\
& \longrightarrow \Lambda^{1}(L) \stackrel{d_{1}}{\longrightarrow} \Lambda^{0}(L) \longrightarrow 0,
\end{aligned}
$$

where the differentials $d_{k}: \Lambda^{k}(L) \rightarrow \Lambda^{k-1}(L)$ are defined by

$$
d_{k}\left(x_{1} \wedge \cdots \wedge x_{k}\right)=\sum_{s<t}(-1)^{s+t}\left[x_{s}, x_{t}\right] \wedge x_{1} \wedge \cdots \wedge \widehat{x_{s}} \wedge \cdots \wedge \widehat{x_{t}} \wedge \cdots \wedge x_{k}
$$

for $k \geq 2, x_{i} \in L$, and $d_{1}=0[\mathrm{CE}],[\mathrm{J}]$.

For each $k \geq 0$ and $\alpha \in \Gamma$, we define $\Lambda^{k}(L)_{\alpha}$ to be the subspace of $\Lambda^{k}(L)$ spanned by the vectors of the form $x_{1} \wedge \cdots \wedge x_{k}\left(x_{i} \in L\right)$ such that $\operatorname{deg}\left(x_{1}\right)+\cdots+\operatorname{deg}\left(x_{k}\right)=$ $\alpha$. Then the space $\Lambda^{k}(L)$ has a $\Gamma$-gradation with finite dimensional homogeneous subspaces, and hence we can define the character $\operatorname{ch} \Lambda^{k}(L)$ of $\Lambda^{k}(L)$. Since the homology module $H_{k}(L)$ inherits a $\Gamma$-gradation from $\Lambda^{k}(L)$, we can also define the character $\operatorname{ch} H_{k}(L)$ of $H_{k}(L)$. Therefore, by applying the Euler-Poincaré principle to (1.3), we obtain

$$
\sum_{k=0}^{\infty}(-1)^{k} \operatorname{ch} \Lambda^{k}(L)=\sum_{k=0}^{\infty}(-1)^{k} \operatorname{ch} H_{k}(L)
$$

Let

$$
\Lambda(L)=\sum_{k=0}^{\infty}(-1)^{k} \Lambda^{k}(L)=\mathbf{C} \ominus L \oplus \Lambda^{2}(L) \ominus \Lambda^{3}(L) \oplus \cdots
$$

be the alternating direct sum of the exterior powers $\Lambda^{k}(L)$ of $L$, and define

$$
\operatorname{ch} \Lambda(L)=\sum_{k=0}^{\infty}(-1)^{k} \operatorname{ch} \Lambda^{k}(L)
$$


Since $\Gamma$ is countable, we have an enumeration $\left\{\beta_{1}, \beta_{2}, \beta_{3}, \cdots\right\}$ of $\Gamma$. Let $\beta \in \Gamma$. Then, for each $k \geq 0, \operatorname{dim} \Lambda^{k}(L)_{\beta}$ is equal to

$$
\sum_{\substack{k_{1} \beta_{i_{1}}+\cdots+k_{r} \beta_{i_{r}}=\beta \\
i_{1}<\cdots<i_{r} \\
k_{1}+\cdots+k_{r}=k}}\left(\begin{array}{c}
\operatorname{dim} L_{\beta_{i_{1}}} \\
k_{1}
\end{array}\right) \cdots\left(\begin{array}{c}
\operatorname{dim} L_{\beta_{i_{r}}} \\
k_{r}
\end{array}\right) .
$$

Therefore, we have

$$
\begin{aligned}
& \operatorname{dim} \Lambda(L)_{\beta}=\sum_{k=0}^{\infty}(-1)^{k} \operatorname{dim} \Lambda^{k}(L)_{\beta} \\
& =\sum_{k=0}^{\infty}(-1)^{k} \sum_{\substack{k_{1} \beta_{i_{1}}+\cdots+k_{r} \beta_{i_{r}}=\beta \\
i_{1}<\cdots<i_{r}=k \\
k_{1}+\cdots+k_{r}=k}}\left(\begin{array}{c}
\operatorname{dim} L_{\beta_{i_{1}}} \\
k_{1}
\end{array}\right) \cdots\left(\begin{array}{c}
\operatorname{dim} L_{\beta_{i_{r}}} \\
k_{r}
\end{array}\right) \\
& =\sum_{\substack{k_{1} \beta_{i_{1}}+\cdots+k_{r} \beta_{i_{r}}=\beta \\
i_{1}<\cdots<i_{r}}}(-1)^{k_{1}+\cdots+k_{r}}\left(\begin{array}{c}
\operatorname{dim} L_{\beta_{i_{1}}} \\
k_{1}
\end{array}\right) \cdots\left(\begin{array}{c}
\operatorname{dim} L_{\beta_{i_{r}}} \\
k_{r}
\end{array}\right) .
\end{aligned}
$$

But this is the same as the coefficient of $e^{\beta}$ in

$$
\prod_{\alpha \in \Gamma}\left(1-e^{\alpha}\right)^{\operatorname{dim} L_{\alpha}}
$$

Hence we obtain

$$
\operatorname{ch} \Lambda(L)=\sum_{k=0}^{\infty}(-1)^{k} \operatorname{ch} \Lambda^{k}(L)=\prod_{\alpha \in \Gamma}\left(1-e^{\alpha}\right)^{\operatorname{dim} L_{\alpha}} .
$$

On the other hand, from the right-hand side of (1.5), we define the homology space of the $\Gamma$-graded Lie algebra $L$ to be an alternating direct sum of homology modules:

$$
H=\sum_{k=1}^{\infty}(-1)^{k+1} H_{k}(L)=H_{1}(L) \ominus H_{2}(L) \oplus H_{3}(L) \ominus \cdots .
$$

For $\alpha \in \Gamma$, we define

$$
d(\alpha)=\operatorname{dim} H_{\alpha}=\sum_{k=1}^{\infty}(-1)^{k+1} \operatorname{dim} H_{k}(L)_{\alpha} .
$$

Then we have

$$
\operatorname{ch} H=\sum_{\alpha \in \Gamma} d(\alpha) e^{\alpha}=\sum_{\alpha \in \Gamma}\left(\operatorname{dim} H_{\alpha}\right) e^{\alpha}=\sum_{k=1}^{\infty}(-1)^{k+1} \operatorname{ch} H_{k}(L) .
$$

Therefore, the Euler-Poincaré principle (1.5) can be written as

$$
\prod_{\alpha \in \Gamma}\left(1-e^{\alpha}\right)^{\operatorname{dim} L_{\alpha}}=1-\operatorname{ch} H=1-\sum_{\alpha \in \Gamma} d(\alpha) e^{\alpha} .
$$

We will call (1.10) the denominator identity for the $\Gamma$-graded Lie algebra $L=$ $\bigoplus_{\alpha \in \Gamma} L_{\alpha}$. Indeed, in Section 3 and 4, we will see that the identity (1.10) is a generalization of the usual denominator identity for symmetrizable Kac-Moody algebras and generalized Kac-Moody algebras (cf. [K2], [K3], [B2]). 
Let $P(H)=\left\{\alpha \in \Gamma \mid \operatorname{dim} H_{\alpha} \neq 0\right\}$, and let $P(H)=\left\{\tau_{1}, \tau_{2}, \tau_{3}, \cdots\right\}$ be an enumeration of $P(H)$. We denote by $d(i)=\operatorname{dim} H_{\tau_{i}}$. For $\tau \in \Gamma$, define

$$
T(\tau)=\left\{s=\left(s_{i}\right)_{i \geq 1} \mid s_{i} \in \mathbf{Z}_{\geq 0}, \sum s_{i} \tau_{i}=\tau\right\}
$$

the set of all partitions of $\tau$ into a sum of $\tau_{i}$ 's. By our finiteness condition on the semigroup $\Gamma$, the set $T(\tau)$ must be finite. For $s \in T(\tau)$, we will use the notation $|s|=\sum s_{i}$ and $s !=\prod s_{i}$ ! (cf. [Bo], [Ju1]). Now for $\tau \in \Gamma$, we define a function

$$
W(\tau)=\sum_{s \in T(\tau)} \frac{(|s|-1) !}{s !} \prod d(i)^{s_{i}} .
$$

We will call $W(\tau)$ the Witt partition function. Then, using the formal power series $\log (1-t)=-\sum_{k=1}^{\infty} \frac{t^{k}}{k}$ and Möbius inversion, we obtain a nice closed form formula for $\operatorname{dim} L_{\alpha}(\alpha \in \Gamma)$.

Theorem 1.1. For $\alpha \in \Gamma$, we have

$$
\operatorname{dim} L_{\alpha}=\sum_{d \mid \alpha} \frac{1}{d} \mu(d) W\left(\frac{\alpha}{d}\right)
$$

where $\mu$ is the classical Möbius function, and, for a positive integer $d, d \mid \alpha$ denotes $\alpha=d \tau$ for some $\tau \in \Gamma$, in which case $\alpha / d=\tau$.

Proof. By the denominator identity (1.10), we have

$$
\prod_{\alpha \in \Gamma}\left(1-e^{\alpha}\right)^{-\operatorname{dim} L_{\alpha}}=\frac{1}{1-\operatorname{ch} H}=\frac{1}{1-\sum_{i=1}^{\infty} d(i) e^{\tau_{i}}} .
$$

Using the formal power series $\log (1-t)=-\sum_{k=1}^{\infty} \frac{1}{k} t^{k}$, we obtain from the left-hand side

$$
\begin{aligned}
& \log \left(\prod_{\alpha \in \Gamma}\left(1-e^{\alpha}\right)^{-\operatorname{dim} L_{\alpha}}\right)=-\sum_{\alpha \in \Gamma}\left(\operatorname{dim} L_{\alpha}\right) \log \left(1-e^{\alpha}\right) \\
& =\sum_{\alpha \in \Gamma}\left(\operatorname{dim} L_{\alpha}\right) \sum_{k=1}^{\infty} \frac{1}{k} e^{k \alpha}=\sum_{\alpha \in \Gamma} \sum_{k=1}^{\infty} \frac{1}{k}\left(\operatorname{dim} L_{\alpha}\right) e^{k \alpha}
\end{aligned}
$$

On the other hand, the right-hand side yields

$$
\begin{aligned}
\log \left(\frac{1}{1-\sum_{i=1}^{\infty} d(i) e^{\tau_{i}}}\right) & =-\log \left(1-\sum_{i=1}^{\infty} d(i) e^{\tau_{i}}\right)=\sum_{k=1}^{\infty} \frac{1}{k}\left(\sum_{i=1}^{\infty} d(i) e^{\tau_{i}}\right)^{k} \\
& =\sum_{k=1}^{\infty} \frac{1}{k} \sum_{\substack{s=\left(s_{i}\right) \\
s_{i} \in \mathbf{Z}_{\geq 0}}} \frac{\left(\sum s_{i}\right) !}{\Pi\left(s_{i} !\right)}\left(\prod d(i)^{s_{i}}\right) e^{\sum s_{i} \tau_{i}} \\
& =\sum_{\tau \in \Gamma}\left(\sum_{\substack{\left.\sum s_{i}=k \\
s \in T\right)}} \frac{(|s|-1) !}{s !} \prod d(i)^{s_{i}}\right) e^{\tau} \\
& =\sum_{\tau \in \Gamma} W(\tau) e^{\tau} .
\end{aligned}
$$


Therefore, we have

$$
W(\tau)=\sum_{\tau=k \alpha} \frac{1}{k}\left(\operatorname{dim} L_{\alpha}\right)=\sum_{k \mid \tau} \frac{1}{k} \operatorname{dim} L_{\frac{\tau}{k}} .
$$

Hence, by Möbius inversion, we obtain

$$
\operatorname{dim} L_{\alpha}=\sum_{d \mid \alpha} \frac{1}{d} \mu(d) W\left(\frac{\alpha}{d}\right) .
$$

Remark. In the proof of Theorem 1.1, observe that the Witt partition function $W(\tau)$ is determined by the identity

$$
\sum_{\tau \in \Gamma} W(\tau) e^{\tau}=\log \left(\frac{1}{1-\operatorname{ch} H}\right) .
$$

In many cases, we can use (1.14) directly to obtain simpler expressions of the Witt partition function $W(\tau)$. On the other hand, we will be able to derive a lot of interesting combinatorial identities from various expressions of the Witt partition function $W(\tau)$ (cf. [KaK1]).

\section{Free Lie Algebras}

In this section, we will discuss the applications of our dimension formula (1.13) to free Lie algebras. When applied to free Lie algebras, our dimension formula (1.13) will be called the generalized Witt formula. An extensive study of free Lie algebras and the generalized Witt formula can be found in [KaK1]. Further applications of our dimension formula to free Lie algebras and the product identities for normalized formal power series will be discussed in Section 5 .

We first recall the following fundamental results on the homology of free Lie algebras (cf. [CE], [GL], [JLW]). Let $V$ be a vector space over $\mathbf{C}$, and let $L=\mathcal{F}(V)$ be the free Lie algebra generated by $V$. Since the universal enveloping algebra $U(L)$ of $L$ is the tensor algebra $\mathcal{T}(V)$ of $V$, the following exact sequence is a $U(L)$-free resolution of the trivial $U(L)$-module $\mathbf{C}$ :

$$
0 \longrightarrow \mathcal{T}(V) \otimes V \stackrel{\varphi}{\longrightarrow} \mathcal{T}(V) \stackrel{\psi}{\longrightarrow} \mathbf{C} \longrightarrow 0,
$$

where $\varphi$ is the multiplication map and $\psi$ is the augmentation map. It follows that

$$
\begin{aligned}
& H_{1}(L)=V, \\
& H_{k}(L)=0 \text { for all } k \geq 2 .
\end{aligned}
$$

Therefore the homology space $H$ is equal to

$$
H=\sum_{k=1}^{\infty}(-1)^{k+1} H_{k}(L)=H_{1}(L)=V .
$$


2.1. Generalized Witt formula. Let $V$ be an $r$-dimensional vector space over $\mathbf{C}$ and let $L=\mathcal{F}(V)$ be the free Lie algebra generated by $V$. Then the Lie algebra $L$ has a $\mathbf{Z}_{>0}$-gradation $L=\bigoplus_{n=1}^{\infty} L_{n}$ by setting $\operatorname{deg} v=1$ for all $v \in V$. The dimensions of the homogeneous subspaces $L_{n}$ are given by the Witt formula

$$
\operatorname{dim} L_{n}=\frac{1}{n} \sum_{d \mid n} \mu(d) r^{\frac{n}{d}}
$$

where $\mu$ is the classical Möbius function (cf. [Bo], [J], [S1]). The main ingredient of the proof of the Witt formula is the following identity:

$$
\prod_{n=1}^{\infty}\left(1-q^{n}\right)^{\operatorname{dim} L_{n}}=1-r q
$$

which is a consequence of the Poincaré-Birkhoff-Witt Theorem. Note that we can deduce the above identity directly from (1.10). Hence it can be considered as the denominator identity for the free Lie algebra $L=\bigoplus_{n=1}^{\infty} L_{n}$.

In this paper, we work in a much more general setting. Let $\Gamma$ be a countable abelian semigroup satisfying our finiteness condition given in Section 1 , and let $V=$ $\bigoplus_{\alpha \in \Gamma} V_{\alpha}$ be a $\Gamma$-graded vector space over $\mathbf{C}$ with $\operatorname{dim} V_{\alpha}=d(\alpha)<\infty$ for all $\alpha \in \Gamma$. Consider the free Lie algebra $L=\mathcal{F}(V)$ generated by $V$. For each $\alpha \in \Gamma$, let $L_{\alpha}$ be the subspace of $L$ spanned by all the vectors of the form $\left[\left[\left[\cdots\left[v_{1}, v_{2}\right] \cdots\right] v_{k-1}\right] v_{k}\right]$ $(k \geq 1)$ with $v_{i} \in V$ such that $\operatorname{deg} v_{1}+\cdots+\operatorname{deg} v_{k}=\alpha$. Then the free Lie algebra $L$ has a $\Gamma$-gradation $L=\bigoplus_{\alpha \in \Gamma} L_{\alpha}$. Now we will apply our dimension formula (1.13) to the $\Gamma$-graded free Lie algebra $L$.

By (2.3), we have $H=H_{1}(L)=V$. Hence $\operatorname{dim} H_{\alpha}=\operatorname{dim} V_{\alpha}=d(\alpha)$ for all $\alpha \in \Gamma$, and $P(H)=P(V)=\left\{\alpha \in \Gamma \mid \operatorname{dim} V_{\alpha}=d(\alpha) \neq 0\right\}$. Therefore, the denominator identity for the $\Gamma$-graded free Lie algebra $L$ is the same as

$$
\prod_{\alpha \in \Gamma}\left(1-e^{\alpha}\right)^{\operatorname{dim} L_{\alpha}}=1-\operatorname{ch} V=1-\sum_{\alpha \in \Gamma} d(\alpha) e^{\alpha}
$$

Let $\left\{\tau_{1}, \tau_{2}, \tau_{3}, \cdots\right\}$ be an enumeration of $P(H)=P(V)$, and $d(i)=\operatorname{dim} V_{\tau_{i}}$ for $i=1,2,3, \cdots$. For $\tau \in \Gamma$, we denote by $T(\tau)$ the set of all partitions of $\tau$ into a sum of $\tau_{i}$ 's as defined in (1.11), and let $W(\tau)$ be the Witt partition function as defined in (1.12). Then our dimension formula (1.13) yields the following generalized version of the Witt formula for the $\Gamma$-graded free Lie algebras.

Theorem 2.1 (cf. [Ka1], [Ka2], [KaK1], [Ju1]). Let $V=\bigoplus_{\alpha \in \Gamma} V_{\alpha}$ be a $\Gamma$-graded vector space over $\mathbf{C}$, and let $L=\bigoplus_{\alpha \in \Gamma} L_{\alpha}$ be the free Lie algebra generated by $V$. Then we have

$$
\operatorname{dim} L_{\alpha}=\sum_{d \mid \alpha} \frac{1}{d} \mu(d) W\left(\frac{\alpha}{d}\right)=\sum_{d \mid \alpha} \frac{1}{d} \mu(d) \sum_{s \in T(\alpha / d)} \frac{(|s|-1) !}{s !} \prod d(i)^{s_{i}},
$$

where $\mu$ is the classical Möbius function, and, for a positive integer $d, d \mid \alpha$ denotes $\alpha=d \tau$ for some $\tau \in \Gamma$, in which case $\alpha / d=\tau$.

Remark. The formula (2.5) will be called the generalized Witt formula for the $\Gamma$ graded free Lie algebras.

As a corollary of Theorem 2.1, we can recover the Witt formula immediately. Let $L=\mathcal{F}(V)$ be the free Lie algebra generated by an $r$-dimensional vector space 
$V$. By (2.3), we have $H=H_{1}(L)=V$, and hence $P(H)=P(V)=\{1\}$. Thus, for each $n \geq 1$, since the only partition of $n$ into a sum of 1 's is $n=n \cdot 1$, we have

$$
T(n)=\{s=(n, 0,0, \cdots)\},
$$

and the Witt partition function $W(n)$ simplifies to

$$
W(n)=\frac{(n-1) !}{n !} r^{n}=\frac{1}{n} r^{n} .
$$

Therefore, the generalized Witt formula (2.5) yields:

Corollary 2.2 (cf. [Bo], [J], [S1]). Suppose $V$ is an $r$-dimensional vector space over $\mathbf{C}$, and let $L=\mathcal{F}(V)=\bigoplus_{n=1}^{\infty} L_{n}$ be the free Lie algebra generated by $V$. Then we have

$$
\operatorname{dim} L_{n}=\frac{1}{n} \sum_{d \mid n} \mu(d) r^{\frac{n}{d}} .
$$

Therefore, the denominator identity (2.4) yields a product identity

$$
\prod_{n=1}^{\infty}\left(1-q^{n}\right)^{\frac{1}{n} \sum_{d \mid n} \mu(d) r^{\frac{n}{d}}}=1-r q .
$$

2.2. 1-dimensional generalization. More generally, let $V=\bigoplus_{i=1}^{\infty} V_{i}$ be a $\mathbf{Z}_{>0^{-}}$ graded vector space with $\operatorname{dim} V_{i}=d(i)<\infty$ for all $i \geq 1$, and consider the free Lie algebra $L=\mathcal{F}(V)$ generated by $V$. For $n \geq 1$, let $L_{n}$ be the subspace of $L$ spanned by all the vectors of the form $\left[\left[\left[\cdots\left[v_{1}, v_{2}\right] \cdots\right] v_{k-1}\right] v_{k}\right](k \geq 1)$ with $v_{i} \in V$ such that $\operatorname{deg} v_{1}+\cdots+\operatorname{deg} v_{k}=n$. Then the Lie algebra $L$ has a $\mathbf{Z}_{>0}$-gradation $L=\bigoplus_{n=1}^{\infty} L_{n}$. We will apply the generalized Witt formula (2.5) to this setting. Note that $P(V)=\mathbf{Z}_{>0}=\{1,2,3, \cdots\}$, and for $n \geq 1$, we have

$$
T(n)=\left\{s=\left(s_{i}\right)_{i \geq 1} \mid s_{i} \in \mathbf{Z}_{\geq 0}, \quad \sum i s_{i}=n\right\},
$$

the set of all partitions of $n$ into a sum of positive integers. Hence the Witt partition function $W(n)$ is equal to

$$
W(n)=\sum_{s \in T(n)} \frac{(|s|-1) !}{s !} \prod d(i)^{s_{i}} .
$$

Therefore, by Theorem 2.1, we obtain the following "1-dimensional generalization" of the Witt formula:

Theorem 2.3. Let $V=\bigoplus_{i=1}^{\infty} V_{i}$ be a $\mathbf{Z}_{>0}$-graded vector space over $\mathbf{C}$ with $\operatorname{dim} V_{i}=$ $d(i)<\infty$ for all $i \geq 1$, and let $L=\bigoplus_{n=1}^{\infty} L_{n}$ be the free Lie algebra generated by $V$. Then we have

$$
\operatorname{dim} L_{n}=\sum_{d \mid n} \frac{1}{d} \mu(d) \sum_{s \in T\left(\frac{n}{d}\right)} \frac{(|s|-1) !}{s !} \prod d(i)^{s_{i}} .
$$

Now, we will consider the applications of the dimension formula (2.10) to several special cases. We first consider the case where $d(i)=r \in \mathbf{Z}_{>0}$ for all $i \geq 1$. In this case, the Witt partition function $W(n)$ is equal to

$$
W(n)=\sum_{s \in T(n)} \frac{(|s|-1) !}{s !} r^{|s|},
$$


and

$$
\sum_{n=1}^{\infty} W(n) q^{n}=\log \frac{1}{1-\operatorname{ch} V}=\log \frac{1}{1-\left(r q+r q^{2}+r q^{3}+\cdots\right)} .
$$

Note that, for any complex number $r \in \mathbf{C}$, we have

$$
\begin{aligned}
& \log \frac{1}{1-\left(r q+r q^{2}+\cdots\right)}=\sum_{k=1}^{\infty} \frac{1}{n}\left(r q+r q^{2}+\cdots\right)^{k} \\
& =\sum_{n=1}^{\infty}\left(\sum_{s \in T(n)} \frac{(|s|-1) !}{s !} r^{|s|}\right) q^{n} .
\end{aligned}
$$

On the other hand, using the formal power series $\frac{1}{1-q}=1+q+q^{2}+\cdots$, we obtain

$$
\begin{aligned}
& \log \frac{1}{1-\left(r q+r q^{2}+\cdots\right)}=\log \frac{1}{1-\frac{r q}{1-q}} \\
& =\log \frac{1-q}{1-(r+1) q}=\log (1-q)-\log (1-(r+1) q) \\
& =\sum_{n=1}^{\infty} \frac{1}{n}\left[(r+1)^{n}-1\right] q^{n} .
\end{aligned}
$$

Therefore, we obtain a combinatorial identity:

$$
\sum_{s \in T(n)} \frac{(|s|-1) !}{s !} r^{|s|}=\frac{1}{n}\left[(r+1)^{n}-1\right] \text { for all } r \in \mathbf{C}, n \in \mathbf{Z}_{>0} .
$$

For example, if $r=1$, we get

$$
\sum_{s \in T(n)} \frac{(|s|-1) !}{s !}=\frac{1}{n}\left(2^{n}-1\right)
$$

and if $r=-1$, we get

$$
\sum_{s \in T(n)} \frac{(|s|-1) !}{s !}(-1)^{|s|}=-\frac{1}{n} .
$$

Moreover, if $r=-1+\zeta$, where $\zeta$ is a primitive $n$th root of unity, then we obtain

$$
\sum_{s \in T(n)} \frac{(|s|-1) !}{s !}(-1+\zeta)^{|s|}=0 .
$$

By (2.12), the Witt partition function $W(n)$ is simplified to

$$
W(n)=\frac{1}{n}\left[(r+1)^{n}-1\right] \quad\left(r \in \mathbf{Z}_{>0}\right)
$$

Combining (2.13) with the dimension formula (2.10), we obtain:

Proposition 2.4. Let $V=\bigoplus_{i=1}^{\infty} V_{i}$ be a $\mathbf{Z}_{>0}$-graded vector space over $\mathbf{C}$ with $\operatorname{dim} V_{i}=r \in \mathbf{Z}_{>0}$ for all $i \geq 1$, and let $L=\bigoplus_{n=1}^{\infty} L_{n}$ be the free Lie algebra 
generated by $V$. Then we have

$$
\begin{aligned}
\operatorname{dim} L_{n} & =\frac{1}{n} \sum_{d \mid n} \mu(d)\left[(r+1)^{\frac{n}{d}}-1\right] \\
& = \begin{cases}\frac{1}{n} \sum_{d \mid n} \mu(d)(r+1)^{\frac{n}{d}} & \text { if } n \neq 1 \\
r & \text { if } n=1 .\end{cases}
\end{aligned}
$$

Therefore, the denominator identity (2.4) yields a product identity

$$
\begin{aligned}
& \prod_{n=1}^{\infty}\left(1-q^{n}\right)^{\frac{1}{n} \sum_{d \mid n} \mu(d)\left[(r+1)^{\frac{n}{d}}-1\right]} \\
& =1-\left(r q+r q^{2}+r q^{3}+\cdots\right) \\
& =1-\frac{r q}{1-q}=\frac{1-(r+1) q}{1-q} .
\end{aligned}
$$

Next, suppose $d(i)=r a^{i}(i \geq 1)$ for some $r, a \in \mathbf{Z}_{>0}$. Then, by (2.12), the Witt partition function $W(n)$ simplifies to

$$
W(n)=\frac{1}{n} a^{n}\left[(r+1)^{n}-1\right]
$$

Combining (2.16) with the dimension formula (2.10), we obtain:

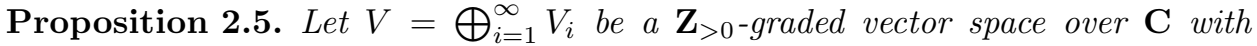
$\operatorname{dim} V_{i}=r a^{i}(i \geq 1)$ for some $r, a \in \mathbf{Z}_{>0}$, and let $L=\bigoplus_{n=1}^{\infty} L_{n}$ be the free Lie algebra generated by $V$. Then we have

$$
\operatorname{dim} L_{n}=\frac{1}{n} \sum_{d \mid n} \mu(d) a^{\frac{n}{d}}\left[(r+1)^{\frac{n}{d}}-1\right] .
$$

Therefore, the denominator identity (2.4) yields a product identity

$$
\begin{aligned}
& \prod_{n=1}^{\infty}\left(1-q^{n}\right)^{\frac{1}{n} \sum_{d \mid n} \mu(d) a^{\frac{n}{d}}\left[(r+1)^{\frac{n}{d}}-1\right]} \\
& =1-\left(r a q+r a^{2} q^{2}+r a^{3} q^{3}+\cdots\right) \\
& =1-\frac{r a q}{1-a q}=\frac{1-(r+1) a q}{1-a q} .
\end{aligned}
$$

Combining (2.18) with (2.15), we obtain the following exchange principle:

Corollary 2.6. For any positive integers a and $r$, we have

$$
\begin{aligned}
& \prod_{n=1}^{\infty}\left(1-a^{n} q^{n}\right)^{\frac{1}{n} \sum_{d \mid n} \mu(d)\left[(r+1)^{\frac{n}{d}}-1\right]} \\
& =\prod_{n=1}^{\infty}\left(1-q^{n}\right)^{\frac{1}{n} \sum_{d \mid n} \mu(d)\left[(r+1)^{\frac{n}{d}}-1\right] a^{\frac{n}{d}} .}
\end{aligned}
$$

Remark. It is shown in [Ka8] that the identity (2.19) holds for all $a, r \in \mathbf{Z}$. 
Now, let us consider the case where $d(1)=0$ and $d(i)=a(i-1)(i \geq 2)$ for some $a \in \mathbf{Z}_{>0}$. In this case, the Witt partition function $W(n)$ is equal to

$$
W(n)=\sum_{s \in T(n)} \frac{(|s|-1) !}{s !} a^{|s|} \prod(i-1)^{s_{i}}
$$

and

$$
\sum_{n=1}^{\infty} W(n) q^{n}=\log \frac{1}{1-\operatorname{ch} V}=\log \frac{1}{1-\sum_{i=2}^{\infty} a(i-1) q^{i}} .
$$

For any complex number $a \in \mathbf{C}$, we have

$$
\begin{aligned}
& \log \frac{1}{1-\sum_{i=2}^{\infty} a(i-1) q^{i}}=\sum_{k=1}^{\infty} \frac{1}{k}\left(\sum_{i=2}^{\infty} a(i-1) q^{i}\right)^{k} \\
& =\sum_{n=1}^{\infty}\left[\sum_{s \in T(n)} \frac{(|s|-1) !}{s !} a^{|s|} \prod(i-1)^{s_{i}}\right] q^{n} .
\end{aligned}
$$

On the other hand, using the formal power series $\frac{1}{(1-q)^{2}}=1+2 q+3 q^{2}+\cdots$, we obtain

$$
\begin{aligned}
& \log \frac{1}{1-\sum_{i=2}^{\infty} a(i-1) q^{i}}=\log \frac{1}{1-\frac{a q^{2}}{(1-q)^{2}}}=\log \frac{(1-q)^{2}}{(1-q)^{2}-a q^{2}} \\
& =\sum_{n=1}^{\infty} \frac{1}{n}\left[(1+\sqrt{a})^{n}+(1-\sqrt{a})^{n}-2\right] q^{n},
\end{aligned}
$$

which yields a combinatorial identity

$$
\begin{gathered}
\sum_{s \in T(n)} \frac{(|s|-1) !}{s !} a^{|s|} \prod(i-1)^{s_{i}}=\frac{1}{n}\left((1+\sqrt{a})^{n}+(1-\sqrt{a})^{n}-2\right) \\
=\frac{2}{n} \sum_{k=1}^{\left[\frac{n}{2}\right]}\left(\begin{array}{c}
n \\
2 k
\end{array}\right) a^{k} \text { for all } a \in \mathbf{C} .
\end{gathered}
$$

It follows that

$$
W(n)=\frac{1}{n}\left[(1+\sqrt{a})^{n}+(1-\sqrt{a})^{n}-2\right] \quad \text { for } a \in \mathbf{Z}_{>0} .
$$

Now, combining (2.22) with the dimension formula (2.10), we obtain:

Proposition 2.7. Let $V=\bigoplus_{i=1}^{\infty} V_{i}$ be a $\mathbf{Z}_{>0}$-graded vector space over $\mathbf{C}$ with $\operatorname{dim} V_{1}=0$ and $\operatorname{dim} V_{i}=a(i-1)(i \geq 2)$ for some $a \in \mathbf{Z}_{>0}$, and let $L=\bigoplus_{n=1}^{\infty} L_{n}$ be the free Lie algebra generated by $V$. Then we have

$$
\begin{aligned}
\operatorname{dim} L_{n} & =\frac{1}{n} \sum_{d \mid n} \mu(d)\left[(1+\sqrt{a})^{\frac{n}{d}}+(1-\sqrt{a})^{\frac{n}{d}}-2\right] \\
& = \begin{cases}\frac{1}{n} \sum_{d \mid n} \mu(d)\left[(1+\sqrt{a})^{\frac{n}{d}}+(1-\sqrt{a})^{\frac{n}{d}}\right] & \text { if } n \neq 1, \\
0 & \text { if } n=1 .\end{cases}
\end{aligned}
$$


Therefore, the denominator identity (2.4) yields a product identity

$$
\begin{aligned}
\prod_{n=1}^{\infty} & \left(1-q^{n}\right)^{\frac{1}{n} \sum_{d \mid n} \mu(d)\left[(1+\sqrt{a})^{\frac{n}{d}}+(1-\sqrt{a})^{\frac{n}{d}}-2\right]} \\
& =1-\sum_{i=2}^{\infty} a(i-1) q^{i}=1-\frac{a q^{2}}{(1-q)^{2}} \\
& =\frac{(1-q)^{2}-a q^{2}}{(1-q)^{2}} .
\end{aligned}
$$

Remark. When $a=2, \varepsilon=1+\sqrt{2}$ is the fundamental unit of $\mathbf{Q}(\sqrt{2})$ and (2.24) can be rewritten as

$$
\prod_{n=1}^{\infty}\left(1-q^{n}\right)^{\frac{1}{n} \sum_{d \mid n} \mu(d) \operatorname{Tr}\left(\varepsilon^{\frac{n}{d}}\right)}=1-2 q-q^{2}
$$

where $\operatorname{Tr}$ is the trace map from $\mathbf{Q}(\sqrt{2})$ to $\mathbf{Q}$.

Finally, suppose $d(1)=0$ and $d(i)=a \alpha^{2}(i-1) \beta^{i-2}(i \geq 2)$ for $a, \alpha, \beta \in \mathbf{Z}_{>0}$. In this case, the Witt partition function $W(n)$ is equal to

$$
W(n)=\beta^{n} \sum_{s \in T(n)} \frac{(|s|-1) !}{s !}\left(\frac{a \alpha^{2}}{\beta^{2}}\right)^{|s|} \prod(i-1)^{s_{i}},
$$

and

$$
\sum_{n=1}^{\infty} W(n) q^{n}=\log \frac{1}{1-\operatorname{ch} V}=\log \frac{1}{1-\sum_{i=2}^{\infty} a \alpha^{2}(i-1) \beta^{i-2} q^{i}} .
$$

For any complex numbers $a, \alpha, \beta \in \mathbf{C}$, we have

$$
\begin{aligned}
& \log \frac{1}{1-\sum_{i=2}^{\infty} a \alpha^{2}(i-1) \beta^{i-2} q^{i}}=\sum_{k=1}^{\infty} \frac{1}{k}\left(\sum_{k=2}^{\infty} a \alpha^{2}(i-1) \beta^{i-2} q^{i}\right)^{k} \\
& =\sum_{n=1}^{\infty}\left[\beta^{n} \sum_{s \in T(n)} \frac{(|s|-1) !}{s !}\left(\frac{a \alpha^{2}}{\beta^{2}}\right)^{|s|} \prod(i-1)^{s_{i}}\right] q^{n} .
\end{aligned}
$$

On the other hand, using the formal power series $\frac{1}{(1-q)^{2}}=1+2 q+3 q^{2}+\cdots$, we obtain

$$
\begin{aligned}
& \log \frac{1}{1-\sum_{i=2}^{\infty} a \alpha^{2}(i-1) \beta^{i-2} q^{i}} \\
& =\log \frac{1}{1-\frac{a \alpha^{2} q^{2}}{(1-\beta q)^{2}}}=\log \frac{(1-\beta q)^{2}}{(1-\beta q)^{2}-a \alpha^{2} q^{2}} \\
& =\sum_{n=1}^{\infty} \frac{1}{n}\left[(\beta+\alpha \sqrt{a})^{n}+(\beta-\alpha \sqrt{a})^{n}-2 \beta^{n}\right] q^{n},
\end{aligned}
$$


which yields a combinatorial identity

$$
\begin{aligned}
& \beta^{n} \sum_{s \in T(n)} \frac{(|s|-1) !}{s !}\left(\frac{a \alpha^{2}}{\beta^{2}}\right)^{|s|} \prod(i-1)^{s_{i}} \\
& =\frac{1}{n}\left[(\beta+\alpha \sqrt{a})^{n}+(\beta-\alpha \sqrt{a})^{n}-2 \beta^{n}\right] \text { for all } a, \alpha, \beta \in \mathbf{C} .
\end{aligned}
$$

Hence we have

$$
W(n)=\frac{1}{n}\left[(\beta+\alpha \sqrt{a})^{n}+(\beta-\alpha \sqrt{a})^{n}-2 \beta^{n}\right] \quad(a, \alpha, \beta \in \mathbf{Z}) .
$$

Now, combining (2.28) with the dimension formula (2.10), we obtain:

Proposition 2.8. Let $V=\bigoplus_{i=1}^{\infty} V_{i}$ be a $\mathbf{Z}_{>0}$-graded vector space over $\mathbf{C}$ with $\operatorname{dim} V_{1}=0$ and $\operatorname{dim} V_{i}=a \alpha^{2}(i-1) \beta^{i-2}(i \geq 2)$ for some $a, \alpha, \beta \in \mathbf{Z}_{>0}$, and let $L=\bigoplus_{n=1}^{\infty} L_{n}$ be the free Lie algebra generated by $V$. Then we have

$$
\operatorname{dim} L_{n}=\frac{1}{n} \sum_{d \mid n} \mu(d)\left[(\beta+\alpha \sqrt{a})^{\frac{n}{d}}+(\beta-\alpha \sqrt{a})^{\frac{n}{d}}-2 \beta^{\frac{n}{d}}\right] .
$$

Therefore, the denominator identity (2.4) yields a product identity

$$
\begin{aligned}
\prod_{n=1}^{\infty} & \left(1-q^{n}\right)^{\frac{1}{n} \sum_{d \mid n} \mu(d)\left[(\beta+\alpha \sqrt{a})^{\frac{n}{d}}+(\beta-\alpha \sqrt{a})^{\frac{n}{d}}-2 \beta^{\frac{n}{d}}\right]} \\
& =1-\sum_{i=2}^{\infty} a \alpha^{2}(i-1) \beta^{i-2} q^{i}=1-\frac{a \alpha^{2} q^{2}}{(1-\beta q)^{2}} \\
& =\frac{(1-\beta q)^{2}-a \alpha^{2} q^{2}}{(1-\beta q)^{2}} .
\end{aligned}
$$

Remark. Let $a$ be a square free integer and let $\gamma=\beta+\alpha \sqrt{a} \in \mathbf{Z}[\sqrt{a}]$. Then (2.30) can be rewritten as

$$
\prod_{n=1}^{\infty}\left(1-q^{n}\right)^{\frac{1}{n} \sum_{d \mid n} \mu(d) \operatorname{Tr}\left(\gamma^{\frac{n}{d}}\right)}=1-\operatorname{Tr}(\gamma) q+\mathrm{N}(\gamma) q^{2},
$$

which generalizes (2.25). Here, $\operatorname{Tr}$ and $N$ are the trace and the norm maps from $\mathbf{Q}(\sqrt{a})$ to $\mathbf{Q}$.

2.3. 2-dimensional generalization. We now consider $\left(\mathbf{Z}_{>0} \times \mathbf{Z}_{>0}\right)$-graded vector spaces $V=\bigoplus_{i, j=1}^{\infty} V_{(i, j)}$ with $\operatorname{dim} V_{(i, j)}=d(i, j)<\infty$ for all $i, j \geq 1$. Let $L=\mathcal{F}(V)$ be the free Lie algebra generated by $V$. For $m, n \geq 1$, let $L_{(m, n)}$ be the subspace of $L$ spanned by all the vectors of the form $\left[\left[\left[\cdots\left[v_{1}, v_{2}\right] \cdots\right] v_{k-1}\right] v_{k}\right](k \geq 1)$ with $v_{i} \in V$ such that $\operatorname{deg} v_{1}+\cdots+\operatorname{deg} v_{k}=(m, n)$. Then the Lie algebra $L$ has a $\left(\mathbf{Z}_{>0} \times \mathbf{Z}_{>0}\right)$-gradation $L=\bigoplus_{m, n=1}^{\infty} L_{(m, n)}$. We will apply the generalized Witt formula (2.5) to this setting. Note that

$$
P(V)=\mathbf{Z}_{>0} \times \mathbf{Z}_{>0}=\{(i, j) \mid i, j=1,2,3, \cdots\},
$$

and for $m, n \geq 1$, we have

$$
T(m, n)=\left\{s=\left(s_{i j}\right)_{i, j \geq 1} \mid s_{i, j} \in \mathbf{Z}_{\geq 0}, \quad \sum s_{i j}(i, j)=(m, n)\right\},
$$


which is the set of all partitions of $(m, n)$ into a sum of ordered pairs of positive integers. Hence the Witt partition function $W(m, n)$ is equal to

$$
W(m, n)=\sum_{s \in T(m, n)} \frac{(|s|-1) !}{s !} \prod d(i, j)^{s_{i j}},
$$

where $|s|=\sum s_{i j}$ and $s !=\prod s_{i j}$ !. Therefore, the generalized Witt formula (2.5) yields the following "2-dimensional generalization" of the Witt formula:

Theorem 2.9. Let $V=\bigoplus_{i, j=1}^{\infty} V_{(i, j)}$ be a $\left(\mathbf{Z}_{>0} \times \mathbf{Z}_{>0}\right)$-graded vector space over C with $\operatorname{dim} V_{(i, j)}=d(i, j)<\infty$ for all $i, j \geq 1$, and let $L=\bigoplus_{m, n=1}^{\infty} L_{(m, n)}$ be the free Lie algebra generated by $V$. Then we have

$$
\operatorname{dim} L_{(m, n)}=\sum_{d \mid(m, n)} \frac{1}{d} \mu(d) \sum_{s \in T\left(\frac{m}{d}, \frac{n}{d}\right)} \frac{(|s|-1) !}{s !} \prod d(i, j)^{s^{s_{j}}} .
$$

Now, we will consider the applications of the dimension formula (2.34) to various special cases. We first consider the case where $d(i, j)=r \in \mathbf{Z}_{>0}$ for all $i, j \geq 1$. In this case, the Witt partition function $W(m, n)$ is equal to

$$
W(m, n)=\sum_{s \in T(m, n)} \frac{(|s|-1) !}{s !} r^{|s|}
$$

and

$$
\sum_{m, n=1}^{\infty} W(m, n) p^{m} q^{n}=\log \frac{1}{1-\operatorname{ch} V}=\log \frac{1}{1-\sum_{i, j=1}^{\infty} r p^{i} q^{j}}
$$

Note that, for any complex number $r \in \mathbf{C}$, we have

$$
\begin{aligned}
& \log \frac{1}{1-\sum_{i, j=1}^{\infty} r p^{i} q^{j}}=\sum_{k=1}^{\infty} \frac{1}{k}\left(\sum_{i, j=1}^{\infty} r p^{i} q^{j}\right)^{k} \\
& =\sum_{m, n=1}^{\infty}\left[\sum_{s \in T(m, n)} \frac{(|s|-1) !}{s !} r^{|s|}\right] p^{m} q^{n} .
\end{aligned}
$$

On the other hand, using the formal power series $\frac{1}{1-q}=1+q+q^{2}+\cdots$, we obtain

$$
\begin{aligned}
\log \frac{1}{1-\sum_{i, j=1}^{\infty} r p^{i} q^{j}} & =\log \frac{1}{1-\frac{r p q}{(1-p)(1-q)}}=\log \frac{(1-p)(1-q)}{1-(p+q+(r-1) p q)} \\
& =\sum_{m, n=1}^{\infty}\left[\sum_{c=0}^{\min (m, n)} \frac{(m+n-c-1) !}{(m-c) !(n-c) ! c !}(r-1)^{c}\right] p^{m} q^{n} .
\end{aligned}
$$

Therefore, we obtain a combinatorial identity

$$
\sum_{s \in T(m, n)} \frac{(|s|-1) !}{s !} r^{|s|}=\sum_{c=0}^{\min (m, n)} \frac{(m+n-c-1) !}{(m-c) !(n-c) ! c !}(r-1)^{c} \text { for all } r \in \mathbf{C}
$$


For example, if $r=0$, we get

$$
\sum_{c=0}^{\min (m, n)} \frac{(m+n-c-1) !}{(m-c) !(n-c) ! c !}(-1)^{c}=0 .
$$

Hence, the Witt partition function $W(m, n)$ is equal to

$$
W(m, n)=\sum_{c=0}^{\min (m, n)} \frac{(m+n-c-1) !}{(m-c) !(n-c) ! c !}(r-1)^{c} \quad\left(r \in \mathbf{Z}_{>0}\right) .
$$

Combining (2.35) and (2.37) with the dimension formula (2.34), we obtain:

Proposition 2.10. Let $V=\bigoplus_{i, j=1}^{\infty} V_{(i, j)}$ be a $\left(\mathbf{Z}_{>0} \times \mathbf{Z}_{>0}\right)$-graded vector space over $\mathbf{C}$ with $\operatorname{dim} V_{(i, j)}=r \in \mathbf{Z}_{>0}$ for all $i, j \geq 1$, and let $L=\bigoplus_{m, n=1}^{\infty} L_{(m, n)}$ be the free Lie algebra generated by $V$. Then we have

$$
\begin{aligned}
& \operatorname{dim} L_{(m, n)}=\sum_{d \mid(m, n)} \frac{1}{d} \mu(d) \sum_{s \in T\left(\frac{m}{d}, \frac{n}{d}\right)} \frac{(|s|-1) !}{s !} r^{|s|} \\
& =\sum_{d \mid(m, n)} \frac{1}{d} \mu(d) \sum_{c=0}^{\min \left(\frac{m}{d}, \frac{n}{d}\right)} \frac{\left(\frac{m}{d}+\frac{n}{d}-c-1\right) !}{\left(\frac{m}{d}-c\right) !\left(\frac{n}{d}-c\right) ! c !}(r-1)^{c} .
\end{aligned}
$$

Therefore, by letting

$$
S_{r}(m, n)=\sum_{c=0}^{\min (m, n)} \frac{(m+n-c-1) !}{(m-c) !(n-c) ! c !}(r-1)^{c} \quad\left(r, m, n \in \mathbf{Z}_{>0}\right),
$$

the denominator identity (2.4) yields a product identity

$$
\begin{aligned}
& \prod_{m, n=1}^{\infty}\left(1-p^{m} q^{n}\right)^{\sum_{d \mid(m, n)} \frac{1}{d} \mu(d) S_{r}\left(\frac{m}{d}, \frac{n}{d}\right)} \\
= & 1-\sum_{i, j=1}^{\infty} r p^{i} q^{j}=1-\frac{r p q}{(1-p)(1-q)} \\
= & \frac{1-[p+q+(r-1) p q]}{(1-p)(1-q)} .
\end{aligned}
$$

Next, we consider the case where $d(i, j)=r a^{i} b^{j}(i, j \geq 1)$ for some positive integers $r, a$, and $b$. In this case, by the identity (2.36), the Witt partition function $W(m, n)$ is equal to

$$
\begin{aligned}
W(m, n) & =a^{m} b^{n}\left(\sum_{s \in T(m, n)} \frac{(|s|-1) !}{s !} r^{|s|}\right) \\
& =a^{m} b^{n} \sum_{c=0}^{\min (m, n)} \frac{(m+n-c-1) !}{(m-c) !(n-c) ! c !}(r-1)^{c} .
\end{aligned}
$$

Combining (2.41) with the dimension formula (2.34), we obtain:

Proposition 2.11. Let $V=\bigoplus_{i, j=1}^{\infty} V_{(i, j)}$ be a $\left(\mathbf{Z}_{>0} \times \mathbf{Z}_{>0}\right)$-graded vector space over $\mathbf{C}$ with $\operatorname{dim} V_{(i, j)}=r a^{i} b^{j}(i, j \geq 1)$ for some positive integers $r$, a, and $b$, and 
let $L=\bigoplus_{m, n=1}^{\infty} L_{(m, n)}$ be the free Lie algebra generated by $V$. Then we have

$$
\begin{aligned}
& \operatorname{dim} L_{(m, n)}=\sum_{d \mid(m, n)} \frac{1}{d} \mu(d) a^{\frac{m}{d}} b^{\frac{n}{d}} \sum_{s \in T\left(\frac{m}{d}, \frac{n}{d}\right)} \frac{(|s|-1) !}{s !} r^{|s|} \\
& =\sum_{d \mid(m, n)} \frac{1}{d} \mu(d) a^{\frac{m}{d}} b^{\frac{n}{d}} \sum_{c=0}^{\min \left(\frac{m}{d}, \frac{n}{d}\right)} \frac{\left(\frac{m}{d}+\frac{n}{d}-c-1\right) !}{\left(\frac{m}{d}-c\right) !\left(\frac{n}{d}-c\right) ! c !}(r-1)^{c} .
\end{aligned}
$$

Therefore, using (2.39), the denominator identity (2.4) yields a product identity

$$
\begin{aligned}
\prod_{m, n=1}^{\infty} & \left(1-p^{m} q^{n}\right)^{\sum_{d \mid(m, n)} \frac{1}{d} \mu(d) a^{\frac{m}{d}} b^{\frac{n}{d}} S_{r}\left(\frac{m}{d}, \frac{n}{d}\right)} \\
& =1-\sum_{i, j=1}^{\infty} r a^{i} b^{j} p^{i} q^{j}=1-\frac{r a b p q}{(1-a p)(1-b q)} \\
& =\frac{1-[a p+b q+(r-1) a b p q]}{(1-a p)(1-b q)} .
\end{aligned}
$$

Comparing (2.40) and (2.43), we obtain the following double exchange principle which is an analogue of (2.19):

Corollary 2.12. For any positive integers $r, a$, and $b$, we have

$$
\begin{aligned}
& \prod_{m, n=1}^{\infty}\left(1-a^{m} b^{n} p^{m} q^{n}\right)^{\sum_{d \mid(m, n)} \frac{1}{d} \mu(d) S_{r}\left(\frac{m}{d}, \frac{n}{d}\right)} \\
& =\prod_{m, n=1}^{\infty}\left(1-p^{m} q^{n}\right)^{\sum_{d \mid(m, n)} \frac{1}{d} \mu(d) a^{\frac{m}{d}} b^{\frac{n}{d}} S_{r}\left(\frac{m}{d}, \frac{n}{d}\right)} .
\end{aligned}
$$

Remark. It is shown in [Ka8] that the identity (2.44) holds for all $a, b, r \in \mathbf{Z}$.

Finally, suppose $d(i, j)=a(i-1)(j-1) \alpha^{i} \beta^{j}(i, j \geq 2)$ for some positive integers $a, \alpha$, and $\beta$. Then the Witt partition function $W(m, n)$ is equal to

$$
W(m, n)=\alpha^{m} \beta^{n}\left(\sum_{s \in T(m, n)} \frac{(|s|-1) !}{s !} a^{|s|} \prod(i-1)^{s_{i j}}(j-1)^{s_{i j}}\right),
$$

and

$$
\begin{aligned}
& \sum_{m, n=1}^{\infty} W(m, n) p^{m} q^{n}=\log \frac{1}{1-\operatorname{ch} V} \\
& =\log \left(\frac{1}{1-\sum_{i, j=2}^{\infty} a(i-1)(j-1) \alpha^{i} \beta^{j} p^{i} q^{j}}\right) .
\end{aligned}
$$


For any complex numbers $a, \alpha, \beta \in \mathbf{C}$, we have

$$
\begin{gathered}
\log \left(\frac{1}{1-\sum_{i, j=2}^{\infty} a(i-1)(j-1) \alpha^{i} \beta^{j} p^{i} q^{j}}\right) \\
=\sum_{k=1}^{\infty} \frac{1}{k}\left(\sum_{i, j=2}^{\infty} a(i-1)(j-1) \alpha^{i} \beta^{j} p^{i} q^{j}\right)^{k} \\
=\sum_{m, n=1}^{\infty} \alpha^{m} \beta^{n}\left[\sum_{c=0}^{\min (m, n)} \frac{(m+n-c-1) !}{(m-c) !(n-c) ! c !}\left((\sqrt{a}-1)^{c}+(-1)^{c}(\sqrt{a}+1)^{c}\right)\right] p^{m} q^{n} .
\end{gathered}
$$

On the other hand, using the formal power series $\frac{1}{(1-q)^{2}}=1+2 q+3 q^{2}+\cdots$, we obtain

$$
\begin{gathered}
\log \frac{1}{1-\sum_{i, j=2}^{\infty} a(i-1)(j-1) \alpha^{i} \beta^{j} p^{i} q^{j}}=\log \frac{1}{1-\frac{a(\alpha p)^{2}(\beta q)^{2}}{(1-\alpha p)^{2}(1-\beta q)^{2}}} \\
=\log \frac{(1-\alpha p)^{2}(1-\beta q)^{2}}{(1-\alpha p)^{2}(1-\beta q)^{2}-a(\alpha p)^{2}(\beta q)^{2}} \\
=\sum_{m, n=1}^{\infty} \alpha^{m} \beta^{n}\left[\sum_{c=0}^{\min (m, n)} \frac{(m+n-c-1) !}{(m-c) !(n-c) ! c !}\left((\sqrt{a}-1)^{c}+(-1)^{c}(\sqrt{a}+1)^{c}\right)\right] p^{m} q^{n} .
\end{gathered}
$$

Therefore, we obtain a combinatorial identity

$$
\begin{aligned}
& \sum_{s \in T(m, n)} \frac{(|s|-1) !}{s !} a^{|s|} \prod(i-1)^{s_{i j}}(j-1)^{s_{i j}} \\
= & \sum_{c=0}^{\min (m, n)} \frac{(m+n-c-1) !}{(m-c) !(n-c) ! c !}\left[(\sqrt{a}-1)^{c}+(-1)^{c}(\sqrt{a}+1)^{c}\right]
\end{aligned}
$$

for all $a \in \mathbf{C}$. For example, if $a=1$, we get

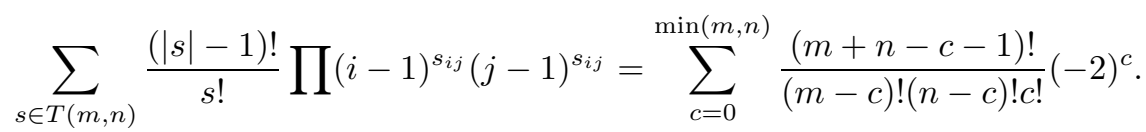

Hence, the Witt partition function $W(m, n)$ is equal to

$$
\begin{aligned}
W(m, n)=\alpha^{m} \beta^{n} & \sum_{c=0}^{\min (m, n)} \frac{(m+n-c-1) !}{(m-c) !(n-c) ! c !} \\
& \times\left[(\sqrt{a}-1)^{c}+(-1)^{c}(\sqrt{a}+1)^{c}\right] .
\end{aligned}
$$

Combining (2.45) and (2.47) with the dimension formula (2.34), we obtain:

Proposition 2.13. Let $V=\bigoplus_{i, j=1}^{\infty} V_{(i, j)}$ be a $\left(\mathbf{Z}_{>0} \times \mathbf{Z}_{>0}\right)$-graded vector space over $\mathbf{C}$ with $\operatorname{dim} V_{(i, j)}=a(i-1)(j-1) \alpha^{i} \beta^{j}(i, j \geq 1)$ for some positive integers $a$, 
$\alpha$, and $\beta$, and let $L=\bigoplus_{m, n=1}^{\infty} L_{(m, n)}$ be the free Lie algebra generated by $V$. Then we have

$$
\begin{aligned}
& \operatorname{dim} L_{(m, n)}=\sum_{d \mid(m, n)} \frac{1}{d} \mu(d) \alpha^{\frac{m}{d}} \beta^{\frac{n}{d}} \\
& \times\left(\sum_{s \in T\left(\frac{m}{d}, \frac{n}{d}\right)} \frac{(|s|-1) !}{s !} a^{|s|} \prod(i-1)^{s_{i j}}(j-1)^{s_{i j}}\right) \\
& =\sum_{d \mid(m, n)} \frac{1}{d} \mu(d) \alpha^{\frac{m}{d}} \beta^{\frac{n}{d}} \sum_{c=0}^{\min \left(\frac{m}{d}, \frac{n}{d}\right)} \frac{\left(\frac{m}{d}+\frac{n}{d}-c-1\right) !}{\left(\frac{m}{d}-c\right) !\left(\frac{n}{d}-c\right) ! c !} \\
& \quad \times\left[(\sqrt{a}-1)^{c}+(-1)^{c}(\sqrt{a}+1)^{c}\right] .
\end{aligned}
$$

Therefore, by letting $S(m, n)=\operatorname{dim} L_{(m, n)}$ for all $m, n \geq 1$, the denominator identity (2.4) yields a product identity

$$
\begin{aligned}
\prod_{m, n=1}^{\infty} & \left(1-p^{m} q^{n}\right)^{S(m, n)}=1-\sum_{i, j=1}^{\infty} a(i-1)(j-1) \alpha^{i} \beta^{j} p^{i} q^{j} \\
& =1-\frac{a(\alpha p)^{2}(\beta q)^{2}}{(1-\alpha p)^{2}(1-\beta q)^{2}} \\
& =\frac{(1-\alpha p)^{2}(1-\beta q)^{2}-a(\alpha p)^{2}(\beta q)^{2}}{(1-\alpha p)^{2}(1-\beta q)^{2}} .
\end{aligned}
$$

2.4. Further generalization. Now we will consider the $n$-dimensional generalization of the exchange principles (2.19) and (2.44). Let $V=\bigoplus_{\left(i_{1}, \cdots, i_{n}\right) \in \mathbf{Z}_{>0}^{n}} V_{\left(i_{1}, \cdots, i_{n}\right)}$ be a $\mathbf{Z}_{>0}^{n}$-graded vector space over $\mathbf{C}$ with

$$
\operatorname{dim} V_{\left(i_{1}, \cdots, i_{n}\right)}=d\left(i_{1}, \cdots, i_{n}\right)<\infty \text { for all }\left(i_{1}, \cdots, i_{n}\right) \in \mathbf{Z}_{>0}^{n},
$$

and let $L=\mathcal{F}(V)$ be the free Lie algebra generated by $V$. For $k_{1}, \cdots, k_{n} \in$ $\mathbf{Z}_{>0}$, let $L_{\left(k_{1}, \cdots, k_{n}\right)}$ be the subspace of $L$ spanned by all the vectors of the form $\left[\left[\left[\cdots\left[v_{1}, v_{2}\right] \cdots\right] v_{t-1}\right] v_{t}\right](t \geq 1)$ with $v_{i} \in V$ such that

$$
\operatorname{deg} v_{1}+\cdots+\operatorname{deg} v_{t}=\left(k_{1}, \cdots, k_{n}\right) .
$$

Then the Lie algebra $L$ has a $\mathbf{Z}_{>0}^{n}$-gradation $L=\bigoplus_{\left(k_{1}, \cdots, k_{n}\right) \in \mathbf{Z}_{>0}^{n}} L_{\left(k_{1}, \cdots, k_{n}\right)}$. We will apply the generalized Witt formula (2.5) to this setting. Note that

$$
P(V)=\mathbf{Z}_{>0}^{n}=\left\{\left(i_{1}, \cdots, i_{n}\right) \mid i_{1}, \cdots, i_{n}=1,2,3, \cdots\right\},
$$

and for $\left(k_{1}, \cdots, k_{n}\right) \in \mathbf{Z}_{>0}^{n}$, we have

$$
\begin{aligned}
T\left(k_{1}, \cdots, k_{n}\right)= & \left\{s=\left(s_{i_{1}, \cdots, i_{n}}\right) \mid s_{i_{1}, \cdots, i_{n}} \in \mathbf{Z}_{\geq 0},\right. \\
& \left.\sum s_{i_{1}, \cdots, i_{n}}\left(i_{1}, \cdots, i_{n}\right)=\left(k_{1}, \cdots, k_{n}\right)\right\},
\end{aligned}
$$

which is the set of all partitions of $\left(k_{1}, \cdots, k_{n}\right)$ into a sum of ordered $n$-tuples of positive integers. Hence the Witt partition function $W\left(k_{1}, \cdots, k_{n}\right)$ is equal to

$$
W\left(k_{1}, \cdots, k_{n}\right)=\sum_{s \in T\left(k_{1}, \cdots, k_{n}\right)} \frac{(|s|-1) !}{s !} \prod d\left(i_{1}, \cdots, i_{n}\right)^{s_{i_{1}, \cdots, i_{n}}},
$$

where $|s|=\sum s_{i_{1}, \cdots, i_{n}}$ and $s !=\prod s_{i_{1}, \cdots, i_{n}}$ !. Therefore, the generalized Witt formula (2.5) yields the following " $n$-dimensional generalization" of the Witt formula: 
Theorem 2.14. Let $V=\bigoplus_{\left(i_{1}, \cdots, i_{n}\right) \in \mathbf{Z}_{>0}^{n}} V_{\left(i_{1}, \cdots i_{n}\right)}$ be a $\mathbf{Z}_{>0}^{n}$-graded vector space over $\mathbf{C}$ with $\operatorname{dim} V_{\left(i_{1}, \cdots, i_{n}\right)}=d\left(i_{1}, \cdots, i_{n}\right)<\infty$ for all $\left(i_{1}, \cdots, i_{n}\right) \in \mathbf{Z}_{>0}^{n}$, and let $L=\bigoplus_{\left(k_{1}, \cdots, k_{n}\right) \in \mathbf{Z}_{>0}^{n}} L_{\left(k_{1}, \cdots, k_{n}\right)}$ be the free Lie algebra generated by $V$. Then we have

$$
\begin{aligned}
& \operatorname{dim} L_{\left(k_{1}, \cdots, k_{n}\right)}=\sum_{d \mid\left(k_{1}, \cdots, k_{n}\right)} \frac{1}{d} \mu(d) W\left(\frac{k_{1}}{d}, \cdots, \frac{k_{n}}{d}\right) \\
& =\sum_{d \mid\left(k_{1}, \cdots, k_{n}\right)} \frac{1}{d} \mu(d) \sum_{s \in T\left(\frac{k_{1}}{d}, \cdots, \frac{k_{n}}{d}\right)} \frac{(|s|-1) !}{s !} \prod d\left(i_{1}, \cdots, i_{n}\right)^{s_{i_{1}}, \cdots, i_{n}} .
\end{aligned}
$$

Now we will consider the application of the dimension formula (2.52) to two special cases. We first consider the case where $d\left(i_{1}, \cdots, i_{n}\right)=r \in \mathbf{Z}_{>0}$ for all $\left(i_{1}, \cdots, i_{n}\right) \in \mathbf{Z}_{>0}^{n}$. In this case, the Witt partition function $W\left(k_{1}, \cdots, k_{n}\right)$ is equal to

$$
W\left(k_{1}, \cdots, k_{n}\right)=\sum_{s \in T\left(k_{1}, \cdots, k_{n}\right)} \frac{(|s|-1) !}{s !} r^{|s|}
$$

and

$$
\begin{gathered}
\sum_{k_{1}, \cdots, k_{n}=1}^{\infty} W\left(k_{1}, \cdots, k_{n}\right) q_{1}^{k_{1}} \cdots q_{n}^{k_{n}}=\log \frac{1}{1-\operatorname{ch} V} \\
=\log \frac{1}{1-\sum_{k_{1}, \cdots, k_{n}=1}^{\infty} r q_{1}^{k_{1}} \cdots q_{n}^{k_{n}}} .
\end{gathered}
$$

Hence, by the dimension formula (2.52), we obtain:

Proposition 2.15. Let $V=\bigoplus_{\left(i_{1}, \cdots, i_{n}\right) \in \mathbf{Z}_{>0}^{n}} V_{\left(i_{1}, \cdots, i_{n}\right)}$ be a $\mathbf{Z}_{>0}^{n}$-graded vector space over $\mathbf{C}$ with $\operatorname{dim} V_{\left(i_{1}, \cdots, i_{n}\right)}=r \in \mathbf{Z}_{>0}$ for all $\left(i_{1}, \cdots, i_{n}\right) \in \mathbf{Z}_{>0}^{n}$, and let $L=$ $\bigoplus_{\left(k_{1}, \cdots, k_{n}\right) \in \mathbf{Z}_{>0}^{n}} L_{\left(k_{1}, \cdots, k_{n}\right)}$ be the free Lie algebra generated by $V$. Then we have

$$
\operatorname{dim} L_{\left(k_{1}, \cdots, k_{n}\right)}=\sum_{d \mid\left(k_{1}, \cdots, k_{n}\right)} \frac{1}{d} \mu(d) \sum_{s \in T\left(\frac{k_{1}}{d}, \cdots, \frac{k_{n}}{d}\right)} \frac{(|s|-1) !}{s !} r^{|s|} .
$$

Therefore, by letting

$$
S_{r}\left(k_{1}, \cdots, k_{n}\right)=\sum_{s \in T\left(k_{1}, \cdots, k_{n}\right)} \frac{(|s|-1) !}{s !} r^{|s|},
$$

the denominator identity (2.4) yields a product identity

$$
\begin{aligned}
& \prod_{k_{1}, \cdots, k_{n}=1}^{\infty}\left(1-q_{1}^{k_{1}} \cdots q_{n}^{k_{n}}\right)^{\sum_{d \mid\left(k_{1}, \cdots, k_{n}\right) \frac{1}{d} \mu(d) S_{r}\left(\frac{k_{1}}{d}, \cdots, \frac{k_{n}}{d}\right)}} \\
= & 1-\sum_{i_{1}, \cdots, i_{n}=1}^{\infty} r q_{1}^{i_{1}} \cdots q_{n}^{i_{n}}=1-\frac{r q_{1} \cdots q_{n}}{\left(1-q_{1}\right) \cdots\left(1-q_{n}\right)} .
\end{aligned}
$$

Next, we consider the case where

$$
d\left(i_{1}, \cdots, i_{n}\right)=r a_{1}^{i_{1}} \cdots a_{n}^{i_{n}} \text { for all }\left(i_{1}, \cdots, i_{n}\right) \in \mathbf{Z}_{>0}^{n}
$$


with $r, a_{1}, \cdots, a_{n} \in \mathbf{Z}_{>0}$. In this case, the Witt partition function $W\left(k_{1}, \cdots, k_{n}\right)$ is equal to

$$
W\left(k_{1}, \cdots, k_{n}\right)=a_{1}^{k_{1}} \cdots a_{n}^{k_{n}}\left(\sum_{s \in T\left(k_{1}, \cdots, k_{n}\right)} \frac{(|s|-1) !}{s !} r^{|s|}\right),
$$

and

$$
\begin{aligned}
\sum_{k_{1}, \cdots, k_{n}=1}^{\infty} & W\left(k_{1}, \cdots, k_{n}\right) q_{1}^{k_{1}} \cdots q_{n}^{k_{n}}=\log \frac{1}{1-\operatorname{ch} V} \\
& =\log \frac{1}{1-\sum_{k_{1}, \cdots, k_{n}=1}^{\infty} r a_{1}^{k_{1}} \cdots a_{n}^{k_{n}} q_{1}^{k_{1}} \cdots q_{n}^{k_{n}}} .
\end{aligned}
$$

Hence, by the dimension formula (2.52), we obtain:

Proposition 2.16. Let $V=\bigoplus_{\left(i_{1}, \cdots, i_{n}\right) \in \mathbf{Z}_{>0}^{n}} V_{\left(i_{1}, \cdots, i_{n}\right)}$ be a $\mathbf{Z}_{>0}^{n}$-graded vector space over $\mathbf{C}$ with

$$
\operatorname{dim} V_{\left(i_{1}, \cdots, i_{n}\right)}=r a_{1}^{i_{1}} \cdots a_{n}^{i_{n}} \quad\left(r, a_{1}, \cdots, a_{n} \in \mathbf{Z}_{>0}\right)
$$

for all $\left(i_{1}, \cdots, i_{n}\right) \in \mathbf{Z}_{>0}^{n}$, and let $L=\bigoplus_{\left(k_{1}, \cdots, k_{n}\right) \in \mathbf{Z}_{>0}^{n}} L_{\left(k_{1}, \cdots, k_{n}\right)}$ be the free Lie algebra generated by $V$. Then we have

$$
\begin{aligned}
\operatorname{dim} L_{\left(k_{1}, \cdots, k_{n}\right)} & =\sum_{d \mid\left(k_{1}, \cdots, k_{n}\right)} \frac{1}{d} \mu(d) a_{1}^{\frac{k_{1}}{d}} \cdots a_{n}^{\frac{k_{n}}{d}} \sum_{s \in T\left(\frac{k_{1}}{d}, \cdots, \frac{k_{n}}{d}\right)} \frac{(|s|-1) !}{s !} r^{|s|} \\
& =\sum_{d \mid\left(k_{1}, \cdots, k_{n}\right)} \frac{1}{d} \mu(d) a_{1}^{\frac{k_{1}}{d}} \cdots a_{n}^{\frac{k_{n}}{d}} S_{r}\left(\frac{k_{1}}{d}, \cdots, \frac{k_{n}}{d}\right) .
\end{aligned}
$$

Therefore, the denominator identity (2.4) yields a product identity

$$
\begin{aligned}
& \prod_{k_{1}, \cdots, k_{n}=1}^{\infty}\left(1-q_{1}^{k_{1}} \cdots q_{n}^{k_{n}}\right)^{\sum_{d \mid\left(k_{1}, \cdots, k_{n}\right)} \frac{1}{d} \mu(d) a_{1}^{\frac{k_{1}}{d}} \cdots a_{n}^{\frac{k_{n}}{d}} S_{r}\left(\frac{k_{1}}{d}, \cdots, \frac{k_{n}}{d}\right)} \\
= & 1-\sum_{i_{1}, \cdots, i_{n}=1}^{\infty} r a_{1}^{i_{1}} \cdots a_{n}^{i_{n}} q_{1}^{i_{1}} \cdots q_{n}^{i_{n}}=1-\frac{r a_{1} \cdots a_{n} q_{1} \cdots q_{n}}{\left(1-a_{1} q_{1}\right) \cdots\left(1-a_{n} q_{n}\right)} .
\end{aligned}
$$

Comparing (2.56) and (2.59), we obtain the following $n$-dimensional exchange principle which is a generalization of (2.19) and (2.44):

Corollary 2.17. For any positive integers $r, a_{1}, \cdots, a_{n}$, we have

$$
\begin{aligned}
& \prod_{k_{1}, \cdots, k_{n}=1}^{\infty}\left(1-a_{1}^{k_{1}} \cdots a_{n}^{k_{n}} q_{1}^{k_{1}} \cdots q_{n}^{k_{n}}\right)^{\sum_{d \mid\left(k_{1}, \cdots, k_{n}\right)} \frac{1}{d} \mu(d) S_{r}\left(\frac{k_{1}}{d}, \cdots, \frac{k_{n}}{d}\right)} \\
= & \prod_{k_{1}, \cdots, k_{n}=1}^{\infty}\left(1-q_{1}^{k_{1}} \cdots q_{n}^{k_{n}}\right)^{\sum_{d \mid\left(k_{1}, \cdots, k_{n}\right)} \frac{1}{d} \mu(d) a_{1}^{\frac{k_{1}}{d}} \cdots a_{n}^{\frac{k_{n}}{d}}} S_{r}\left(\frac{k_{1}}{d}, \cdots, \frac{k_{n}}{d}\right) .
\end{aligned}
$$

Remark. It is shown in [Ka8] that the identity (2.60) holds for all $r, a_{1}, \cdots, a_{n} \in \mathbf{Z}$. 


\section{KaC-MOODY ALGEBRAS}

In this section, we will apply our dimension formula (1.13) to symmetrizable KacMoody algebras to derive a closed form root multiplicity formula (Theorem 3.5), which is a generalization of Berman-Moody's formula [BM]. Our root multiplicity formula enables us to study the structure of a symmetrizable Kac-Moody algebra $\mathfrak{g}$ as a representation of the smaller Kac-Moody algebra $\mathfrak{g}_{0}$ contained in $\mathfrak{g}$. We will discuss the applications of our formula to some classes of indefinite Kac-Moody algebras.

3.1. The Weyl-Kac character formula. We begin with some of the basic definitions in Kac-Moody theory [K3]. Let $I$ be a finite index set. An integral matrix $A=\left(a_{i j}\right)_{i, j \in I}$ is called a generalized Cartan matrix if (i) $a_{i i}=2$ for all $i \in I$, (ii) $a_{i j} \leq 0$ for $i \neq j$, (iii) $a_{i j}=0$ implies $a_{j i}=0$. We assume that the generalized Cartan matrix $A$ is symmetrizable, i.e., there is an invertible diagonal matrix $D$ such that $D A$ is symmetric. A realization of $A$ is a triple $\left(\mathfrak{h}, \Pi, \Pi^{\vee}\right)$, where $\mathfrak{h}$ is a complex vector space of dimension $2|I|-\operatorname{rank} A, \Pi=\left\{\alpha_{i} \mid i \in I\right\}$ and $\Pi^{\vee}=\left\{h_{i} \mid i \in I\right\}$ are linearly independent subsets of $\mathfrak{h}^{*}$ and $\mathfrak{h}$, respectively, satisfying $\alpha_{j}\left(h_{i}\right)=a_{i j}$ for $i, j \in I$.

Definition 3.1. The Kac-Moody algebra $\mathfrak{g}=\mathfrak{g}(A)$ associated with a generalized Cartan matrix $A=\left(a_{i j}\right)_{i, j \in I}$ is the Lie algebra generated by the elements $e_{i}, f_{i}$ $(i \in I)$ and $h \in \mathfrak{h}$ with the defining relations:

$$
\begin{aligned}
& {\left[h, h^{\prime}\right]=0 \quad\left(h, h^{\prime} \in \mathfrak{h}\right),} \\
& {\left[h, e_{i}\right]=\alpha_{i}(h) e_{i}, \quad\left[h, f_{j}\right]=-\alpha_{i}(h) f_{i} \quad(i \in I),} \\
& {\left[e_{i}, f_{j}\right]=\delta_{i j} h_{i} \quad(i, j \in I),} \\
& \left(a d e_{i}\right)^{1-a_{i j}}\left(e_{j}\right)=\left(a d f_{i}\right)^{1-a_{i j}}\left(f_{j}\right)=0 \quad(i \neq j) .
\end{aligned}
$$

The elements of $\Pi$ (resp. $\Pi^{\vee}$ ) are called the simple roots (resp. simple coroots) of $\mathfrak{g}$. For each $i \in I$, we define the simple reflection $r_{i} \in G L\left(\mathfrak{h}^{*}\right)$ on $\mathfrak{h}^{*}$ by $r_{i}(\lambda)=$ $\lambda-\lambda\left(h_{i}\right) \alpha_{i}$. The subgroup $W$ of $G L\left(\mathfrak{h}^{*}\right)$ generated by the $r_{i}$ 's $(i \in I)$ is called the Weyl group of $\mathfrak{g}$.

Let $Q=\bigoplus_{i \in I} \mathbf{Z} \alpha_{i}, Q^{+}=\sum_{i \in I} \mathbf{Z}_{\geq 0} \alpha_{i}$, and $Q^{-}=-Q^{+}$. We define a partial ordering $\geq$ on $\mathfrak{h}^{*}$ by $\lambda \geq \mu$ if and only if $\lambda-\mu \in Q^{+}$. The Kac-Moody algebra $\mathfrak{g}=\mathfrak{g}(A)$ has the root space decomposition

$$
\mathfrak{g}=\bigoplus_{\alpha \in Q} \mathfrak{g}_{\alpha}, \quad \text { where } \mathfrak{g}_{\alpha}=\{x \in \mathfrak{g} \mid[h, x]=\alpha(h) x \text { for all } h \in \mathfrak{h}\} .
$$

An element $\alpha \in Q$ is called a root if $\alpha \neq 0$ and $\mathfrak{g}_{\alpha} \neq 0$, and $\operatorname{dim} \mathfrak{g}_{\alpha}$ is called the multiplicity of the root $\alpha$. A root $\alpha>0$ (resp. $\alpha<0$ ) is called positive (resp. negative). All the roots are either positive or negative. We denote by $\Delta, \Delta^{+}$, and $\Delta^{-}$the set of all roots, positive roots, and negative roots, respectively. Let $\mathfrak{g}_{+}=\bigoplus_{\alpha>0} \mathfrak{g}_{\alpha}$ and $\mathfrak{g}_{-}=\bigoplus_{\alpha<0} \mathfrak{g}_{\alpha}$. Then we have the triangular decomposition

$$
\mathfrak{g}=\mathfrak{g}_{-} \oplus \mathfrak{g}_{0} \oplus \mathfrak{g}_{+} .
$$

A $\mathfrak{g}$-module $V$ is called $\mathfrak{h}$-diagonalizable if

$$
V=\bigoplus_{\lambda \in \mathfrak{h}^{*}} V_{\lambda}, \quad \text { where } V_{\lambda}=\{v \in V \mid h \cdot v=\lambda(h) v \text { for all } h \in \mathfrak{h}\} .
$$


If $V_{\lambda} \neq 0$, then $\lambda$ is called a weight of $V$, and $\operatorname{dim} V_{\lambda}$ is called the multiplicity of $\lambda$ in $V$. When $\operatorname{dim} V_{\lambda}<\infty$ for all $\lambda \in \mathfrak{h}^{*}$, we define the character of $V$ to be

$$
\operatorname{ch} V=\sum_{\lambda \in \mathfrak{h}^{*}}\left(\operatorname{dim} V_{\lambda}\right) e^{\lambda},
$$

where $e^{\lambda}$ are the basis elements of the group algebra $\mathbf{C}\left[\mathfrak{h}^{*}\right]$ with the multiplication $e^{\lambda} e^{\mu}=e^{\lambda+\mu}$.

A $\mathfrak{g}$-module $V$ is called a highest weight module with highest weight $\lambda \in \mathfrak{h}^{*}$ if there is a nonzero vector $v \in V$ such that (i) $\mathfrak{g}_{+} \cdot v=0$, (ii) $h \cdot v=\lambda(h) v$ for all $h \in \mathfrak{h}$, (iii) $U(\mathfrak{g}) \cdot v=V$, where $U(\mathfrak{g})$ is the universal enveloping algebra of $\mathfrak{g}$. The vector $v$ is called a highest weight vector. Let $\mathfrak{b}_{+}=\mathfrak{h}+\mathfrak{g}_{+}$be the Borel subalgebra of $\mathfrak{g}$ and $\mathbf{C}_{\lambda}$ be the 1-dimensional $\mathfrak{b}_{+}$-module defined by $\mathfrak{g}_{+} \cdot 1=0, h \cdot 1=\lambda(h) 1$ for all $h \in \mathfrak{h}$. The induced module $M(\lambda)=U(\mathfrak{g}) \otimes_{U\left(\mathfrak{b}_{+}\right)} \mathbf{C}_{\lambda}$ is called the Verma module with highest weight $\lambda$. Every highest weight $\mathfrak{g}$-module with highest weight $\lambda$ is a quotient of $M(\lambda)$. The Verma module $M(\lambda)$ contains a unique maximal submodule $J(\lambda)$, and its irreducible quotient $M(\lambda) / J(\lambda)$ will be denoted by $V(\lambda)$.

If $\lambda$ is dominant integral, i.e., $\lambda\left(h_{i}\right) \in \mathbf{Z}_{\geq 0}$ for all $i \in I$, then the character of $V(\lambda)$ is given by the Weyl-Kac formula:

Proposition 3.2 ([K2], [K3, Theorem 10.4]).

$$
\operatorname{ch} V(\lambda)=\frac{\sum_{w \in W}(-1)^{l(w)} e^{w(\lambda+\rho)-\rho}}{\prod_{\alpha \in \Delta^{-}}\left(1-e^{\alpha}\right)^{\operatorname{dimg}_{\alpha}}},
$$

where $\rho \in \mathfrak{h}^{*}$ is a linear functional satisfying $\rho\left(h_{i}\right)=1$ for all $i \in I$, and $l(w)$ denotes the length of $w$.

When $\lambda=0$, we obtain the usual denominator identity for symmetrizable KacMoody algebras :

$$
\prod_{\alpha \in \Delta^{-}}\left(1-e^{\alpha}\right)^{\operatorname{dim} g_{\alpha}}=\sum_{w \in W}(-1)^{l(w)} e^{w \rho-\rho}
$$

3.2. The root multiplicity formula. Let $J$ be a subset of $I$ and we denote by $\Delta_{J}=\Delta \cap\left(\sum_{j \in J} \mathbf{Z} \alpha_{j}\right), \Delta_{J}^{ \pm}=\Delta_{J} \cap \Delta^{ \pm}$, and $\Delta^{ \pm}(J)=\Delta^{ \pm} \backslash \Delta_{J}^{ \pm}$. Similarly, let $Q_{J}=Q \cap\left(\sum_{j \in J} \mathbf{Z} \alpha_{j}\right), Q_{J}^{ \pm}=Q_{J} \cap Q^{ \pm}$, and $Q^{ \pm}(J)=Q^{ \pm} \backslash Q_{J}^{ \pm}$. Define $\mathfrak{g}_{0}^{(J)}=\mathfrak{h} \oplus\left(\bigoplus_{\alpha \in \Delta_{J}} \mathfrak{g}_{\alpha}\right)$, and $\mathfrak{g}_{ \pm}^{(J)}=\bigoplus_{\alpha \in \Delta^{ \pm}(J)} \mathfrak{g}_{\alpha}$. Then we have the triangular decomposition:

$$
\mathfrak{g}=\mathfrak{g}_{-}^{(J)} \oplus \mathfrak{g}_{0}^{(J)} \oplus \mathfrak{g}_{+}^{(J)},
$$

where $\mathfrak{g}_{0}^{(J)}$ is the Kac-Moody algebra (with an extended Cartan subalgebra) associated with the generalized Cartan matrix $A_{J}=\left(a_{i j}\right)_{i, j \in J}$, and $\mathfrak{g}_{-}^{(J)}$ (resp. $\left.\mathfrak{g}_{+}^{(J)}\right)$ is a direct sum of irreducible highest weight (resp. lowest weight) modules over $\mathfrak{g}_{0}^{(J)}$ [K3, Ch. 10].

Let $W_{J}=\left\langle r_{j} \mid j \in J\right\rangle$ be the subgroup of $W$ generated by the simple reflections $r_{j}(j \in J)$, and let $W(J)=\left\{w \in W \mid w \Delta^{-} \cap \Delta^{+} \subset \Delta^{+}(J)\right\}$. Then $W_{J}$ is the Weyl group of the subalgebra $\mathfrak{g}_{0}^{(J)}$ and $W(J)$ is the set of right coset representatives of $W_{J}$ in $W$. That is, $W=W_{J} W(J)$ (see, for example, [Li]). The following lemma is very useful in actual computation.

Lemma 3.3 (cf. [Ka1], [Ka2], [Li]). Suppose $w=w^{\prime} r_{j}$ and $l(w)=l\left(w^{\prime}\right)+1$. Then $w \in W(J)$ if and only if $w^{\prime} \in W(J)$ and $w^{\prime}\left(\alpha_{j}\right) \in \Delta^{+}(J)$. 
Since $\mathfrak{g}_{\alpha}=0$ for $\alpha \in Q^{-}(J) \backslash \Delta^{-}(J)$, the Lie algebra $\mathfrak{g}_{-}^{(J)}$ is graded by the abelian semigroup $Q^{-}(J)$, which satisfies the finiteness condition in the beginning of Section 1. We will apply our dimension formula to the algebra $\mathfrak{g}_{-}^{(J)}=\bigoplus_{\alpha \in Q^{-}(J)} \mathfrak{g}_{\alpha}$.

Let $\mathbf{C}$ be the trivial $\mathfrak{g}$-module. The homology modules $H_{k}\left(\mathfrak{g}_{-}^{(J)}\right)=H_{k}\left(\mathfrak{g}_{-}^{(J)}, \mathbf{C}\right)$ are determined by the following formula known as Kostant's formula:

Proposition 3.4 ([GL], [Li]).

$$
H_{k}\left(\mathfrak{g}_{-}^{(J)}\right) \cong \bigoplus_{\substack{w \in W(J) \\ l(w)=k}} V_{J}(w \rho-\rho),
$$

where $V_{J}(\mu)$ denotes the irreducible highest weight $\mathfrak{g}_{0}^{(J)}$-module with highest weight $\mu$.

Therefore, the homology space for the algebra $\mathfrak{g}_{-}^{(J)}$ is equal to

$$
\begin{aligned}
H^{(J)} & =\sum_{k=1}^{\infty}(-1)^{k+1} H_{k}\left(\mathfrak{g}_{-}^{(J)}\right)=\sum_{k=1}^{\infty}(-1)^{k+1} \sum_{\substack{w \in W(J) \\
l(w)=k}} V_{J}(w \rho-\rho) \\
& =\sum_{\substack{w \in W(J) \\
l(w) \geq 1}}(-1)^{l(w)+1} V_{J}(w \rho-\rho),
\end{aligned}
$$

and hence the denominator identity for the algebra $\mathfrak{g}_{-}^{(J)}$ is the same as

$$
\prod_{\alpha \in Q^{-}(J)}\left(1-e^{\alpha}\right)^{\operatorname{dimg} g_{\alpha}}=\sum_{w \in W(J)}(-1)^{l(w)} \operatorname{ch} V_{J}(w \rho-\rho) .
$$

In particular, when $J=\phi$, we have $W(J)=W$, and we recover the usual denominator identity (3.7).

Let $P\left(H^{(J)}\right)=\left\{\alpha \in Q^{-}(J) \mid \operatorname{dim} H_{\alpha}^{(J)} \neq 0\right\}=\left\{\tau_{1}, \tau_{2}, \tau_{3}, \cdots\right\}$, and $d(i)=$ $\operatorname{dim} H_{\tau_{i}}^{(J)}$ for $i=1,2,3, \cdots$. For $\tau \in Q^{-}(J)$, we denote by $T^{(J)}(\tau)$ the set of all partitions of $\tau$ into a sum of $\tau_{i}$ 's as defined in (1.11), and let $W^{(J)}(\tau)$ be the Witt partition function as defined in (1.12). Then our dimension formula (1.13) yields a closed form root multiplicity formula for all symmetrizable Kac-Moody algebras.

Theorem 3.5 (cf. [Ka5]). Let $\alpha \in \Delta^{-}(J)$ be a root of a symmetrizable Kac-Moody algebra $\mathfrak{g}$. Then we have

$$
\begin{aligned}
& \operatorname{dimg}_{\alpha}=\sum_{d \mid \alpha} \frac{1}{d} \mu(d) W^{(J)}\left(\frac{\alpha}{d}\right) \\
& =\sum_{d \mid \alpha} \frac{1}{d} \sum_{s \in T^{(J)}\left(\frac{\alpha}{d}\right)} \frac{(|s|-1) !}{s !} \prod d(i)^{s_{i}} .
\end{aligned}
$$

If $J=\phi$, then the homology space is equal to

$$
H^{(\phi)}=\sum_{\substack{w \in W \\ l(w) \geq 1}}(-1)^{l(w)+1} \mathbf{C}_{w \rho-\rho},
$$

and hence $P\left(H^{(\phi)}\right)=\{w \rho-\rho \mid w \in W, l(w) \geq 1\}$. Let $\left\{\tau_{i}=w_{i} \rho-\rho \mid i=1,2,3, \cdots\right\}$ be an enumeration of $P\left(H^{(\phi)}\right)$, and denote by $T^{(\phi)}(\tau)$ the set of all partitions of $\tau$ 
into a sum of $\tau_{i}$ 's. Then $\operatorname{dim} H_{\tau_{i}}^{(\phi)}=(-1)^{l\left(w_{i}\right)+1}$, and our root multiplicity formula (3.12) reduces to Berman-Moody's formula:

Corollary 3.6 ([BM]).

$$
\operatorname{dimg}_{\alpha}=\sum_{d \mid \alpha} \frac{1}{d} \mu(d) \sum_{s \in T^{(\phi)}\left(\frac{\alpha}{d}\right)} \frac{(|s|-1) !}{s !} \prod\left[(-1)^{l\left(w_{i}\right)+1}\right]^{s_{i}} .
$$

3.3. Rank 2 hyperbolic Kac-Moody algebras. Let $A=\left(\begin{array}{cc}2 & -a \\ -b & 2\end{array}\right)$ be a generalized Cartan matrix with $a \geq b$ and $a b>4$. Since $\left(\begin{array}{cc}1 & 0 \\ 0 & \frac{a}{b}\end{array}\right) A=\left(\begin{array}{cc}2 & -a \\ -a & \frac{2 a}{b}\end{array}\right)$, $A$ is symmetrizable, and the Kac-Moody algebra $\mathfrak{g}=\mathfrak{g}(a, b)$ associated with $A$ is of hyperbolic type [K3, Ch.4]. In this subsection, we will apply our root multiplicity formula (3.12) to the rank 2 hyperbolic Kac-Moody algebra $\mathfrak{g}=\mathfrak{g}(a, b)$, and determine its root multiplicities using the Fibonacci-type sequences $\left\{A_{n}\right\}_{n \geq 0}$ and $\left\{B_{n}\right\}_{n \geq 0}$ defined by

$$
\begin{aligned}
& A_{0}=B_{0}=0, \quad A_{1}=B_{1}=1 \\
& A_{n+2}=a B_{n+1}-A_{n}+1 \\
& B_{n+2}=b A_{n+1}-B_{n}+1 \quad(n \geq 0)
\end{aligned}
$$

(see also [K3, Exercise 5.25]).

We first take $J=\phi$. Then $\mathfrak{g}_{0}^{(J)}=\mathbf{C} h_{0} \oplus \mathbf{C} h_{1}$ is the Cartan subalgebra and $W(\phi)=W$, the full Weyl group, which is the free product of two cyclic groups of order 2. Thus we have an explicit description of $W$ :

$$
W=\left\{1, r_{0}\left(r_{1} r_{0}\right)^{j}, r_{1}\left(r_{0} r_{1}\right)^{j},\left(r_{0} r_{1}\right)^{j+1},\left(r_{1} r_{0}\right)^{j+1} \mid j \geq 0\right\},
$$

and the homology space $H^{(\phi)}$ is equal to

$$
\begin{aligned}
H^{(\phi)} & =\sum_{\substack{w \in W \\
l(w) \geq 1}}(-1)^{l(w)+1} \mathbf{C}_{w \rho-\rho} \\
& =\sum_{j \geq 0}\left(\mathbf{C}_{r_{0}\left(r_{1} r_{0}\right)^{j} \rho-\rho} \oplus \mathbf{C}_{r_{1}\left(r_{0} r_{1}\right)^{j} \rho-\rho}\right) \\
& \ominus \sum_{j \geq 0}\left(\mathbf{C}_{\left(r_{0} r_{1}\right)^{j+1} \rho-\rho} \oplus \mathbf{C}_{\left(r_{1} r_{0}\right)^{j+1} \rho-\rho}\right) .
\end{aligned}
$$

By induction, we obtain

$$
\begin{aligned}
& r_{0}\left(r_{1} r_{0}\right)^{j} \rho-\rho=-A_{2 j+1} \alpha_{0}-B_{2 j} \alpha_{1}, \\
& r_{1}\left(r_{0} r_{1}\right)^{j} \rho-\rho=-A_{2 j} \alpha_{0}-B_{2 j+1} \alpha_{1}, \\
& \left(r_{0} r_{1}\right)^{j+1} \rho-\rho=-A_{2 j+2} \alpha_{0}-B_{2 j+1} \alpha_{1}, \\
& \left(r_{1} r_{0}\right)^{j+1} \rho-\rho=-A_{2 j+1} \alpha_{0}-B_{2 j+2} \alpha_{1} .
\end{aligned}
$$


It follows that

$$
\begin{aligned}
H^{(\phi)} & =\sum_{j \geq 0}\left(\mathbf{C}_{-A_{2 j+1} \alpha_{0}-B_{2 j} \alpha_{1}} \oplus \mathbf{C}_{-A_{2 j} \alpha_{0}-B_{2 j+1} \alpha_{1}}\right) \\
& \ominus \sum_{j \geq 0}\left(\mathbf{C}_{-A_{2 j+2} \alpha_{0}-B_{2 j+1} \alpha_{1}} \oplus \mathbf{C}_{-A_{2 j+1} \alpha_{0}-B_{2 j+2} \alpha_{1}}\right) \\
& =\sum_{i=1}^{\infty}(-1)^{i}\left(\mathbf{C}_{-A_{i+1} \alpha_{0}-B_{i} \alpha_{1}} \oplus \mathbf{C}_{-A_{i} \alpha_{0}-B_{i+1} \alpha_{1}}\right) .
\end{aligned}
$$

Therefore, by identifying $-k \alpha_{0}-l \alpha_{1} \in Q^{-}$with $(k, l) \in \mathbf{Z}_{\geq 0} \times \mathbf{Z}_{\geq 0}$, we have

$$
P\left(H^{(\phi)}\right)=\left\{\left(A_{i+1}, B_{i}\right),\left(A_{i}, B_{i+1}\right) \mid i \geq 0\right\}
$$

and

$$
\operatorname{dim} H_{\left(A_{i+1}, B_{i}\right)}^{(\phi)}=\operatorname{dim} H_{\left(A_{i}, B_{i+1}\right)}^{(\phi)}=(-1)^{i} \quad(i \geq 0) .
$$

Hence the denominator identity of the algebra $\mathfrak{g}_{-}^{(\phi)}$ is equal to

$$
\prod_{\substack{m, n \in \mathbf{Z}_{\geq 0} \\(m, n) \neq(0,0)}}\left(1-p^{m} q^{n}\right)^{\operatorname{dimg}_{(m, n)}}=1-\sum_{i=0}^{\infty}(-1)^{i}\left(p^{A_{i+1}} q^{B_{i}}+p^{A_{i}} q^{B_{i+1}}\right),
$$

where $p=e^{-\alpha_{0}}, q=e^{-\alpha_{1}}$. Moreover, the set $T^{(\phi)}$ of all the partitions of $(k, l)$ into a sum of $\left(A_{i+1}, B_{i}\right)$ 's and $\left(A_{j}, B_{j+1}\right)$ 's $(i, j \geq 0)$ is given by

$$
\begin{aligned}
T^{(\phi)}(k, l)= & \left\{(s ; t)=\left(s_{i} ; t_{j}\right)_{i, j \geq 0} \mid s_{i}, t_{j} \in \mathbf{Z}_{\geq 0},\right. \\
& \left.\sum_{i \geq 0} s_{i}\left(A_{i+1}, B_{i}\right)+\sum_{j \geq 0} t_{j}\left(A_{j+1}, B_{j}\right)=(k, l)\right\},
\end{aligned}
$$

and the Witt partition function $W^{(\phi)}(k, l)$ is the same as

$$
W^{(\phi)}(k, l)=\sum_{(s ; t) \in T^{(\phi)}(k, l)} \frac{(|s|+|t|-1) !}{s ! t !}(-1)^{\sum i s_{i}+\sum j t_{j}} .
$$

Therefore, our root multiplicity formula (3.12) yields:

Proposition 3.7. Let $\mathfrak{g}=\mathfrak{g}(a, b)$ be the hyperbolic Kac-Moody algebra associated with the generalized Cartan matrix $A=\left(\begin{array}{cc}2 & -a \\ -b & 2\end{array}\right)$ with $a \geq b$ and $a b>4$. Then, for the root $\alpha=-m \alpha_{0}-n \alpha_{1}$, we have

$$
\begin{aligned}
& \operatorname{dimg}_{\alpha}=\sum_{d \mid(m, n)} \frac{1}{d} \mu(d) W^{(\phi)}\left(\frac{m}{d}, \frac{n}{d}\right) \\
& =\sum_{d \mid(m, n)} \frac{1}{d} \mu(d) \sum_{(s ; t) \in T^{(\phi)}\left(\frac{m}{d}, \frac{n}{d}\right)} \frac{(|s|+|t|-1) !}{s ! t !}(-1)^{\sum i s_{i}+\sum j t_{j}} .
\end{aligned}
$$

Example 3.8. Let $a=4$ and $b=3$. We will compute the multiplicity of the root $\alpha=-5 \alpha_{0}-4 \alpha_{1}$ of the hyperbolic Kac-Moody algebra $\mathfrak{g}=\mathfrak{g}(4,3)$. By the formula (3.24), we have

$$
\operatorname{dimg}(4,3)_{(5,4)}=W^{(\phi)}(5,4) .
$$


The first few terms of the sequences $\left\{A_{n}\right\}_{n \geq 0}$ and $\left\{B_{n}\right\}_{n \geq 0}$ are

$$
\begin{aligned}
& A_{0}=B_{0}=0, \quad A_{1}=B_{1}=1, \quad A_{2}=5, \quad B_{2}=4, \\
& A_{3}=16, \quad B_{3}=15, \cdots .
\end{aligned}
$$

Hence $P\left(H^{(\phi)}\right)=\{(1,0),(0,1),(5,1),(1,4),(16,4),(5,15), \cdots\}$, where

$$
\begin{aligned}
& \operatorname{mult}(1,0)=\operatorname{mult}(0,1)=1, \\
& \operatorname{mult}(5,1)=\operatorname{mult}(1,4)=-1, \\
& \operatorname{mult}(16,4)=\operatorname{mult}(5,15)=1, \cdots .
\end{aligned}
$$

Since $(5,4)=5(1,0)+4(0,1)=4(1,0)+(1,4)=(5,1)+3(0,1)$, we get

$$
W^{(\phi)}(5,4)=\frac{8 !}{5 ! 4 !}+\frac{4 !}{4 !}(-1)+\frac{3 !}{3 !}(-1)=12 .
$$

Hence $\operatorname{dimg}(4,3)_{(5,4)}=W^{(\phi)}(5,4)=12$

Now we take $J=\{1\}$. Then $\mathfrak{g}_{0}^{(J)}=\left\langle e_{1}, f_{1}, h_{1}\right\rangle \oplus \mathbf{C} h_{0} \cong \operatorname{sl}(2, \mathbf{C}) \oplus \mathbf{C} h_{0}, W(J)=$ $\left\{1, r_{0}\left(r_{1} r_{0}\right)^{j},\left(r_{0} r_{1}\right)^{j+1} \mid j \geq 0\right\}$, and

$$
\begin{aligned}
H^{(J)} & =\sum_{\substack{w \in W(J) \\
l(w) \geq 1}}(-1)^{l(w)+1} V_{J}(w \rho-\rho) \\
& =\sum_{j=0}^{\infty}\left(V_{J}\left(-A_{2 j+1} \alpha_{0}-B_{2 j} \alpha_{1}\right) \ominus V_{J}\left(-A_{2 j+2} \alpha_{0}-B_{2 j+1} \alpha_{1}\right)\right) \\
& =\sum_{i=0}^{\infty}(-1)^{i} V_{J}\left(-A_{i+1} \alpha_{0}-B_{i} \alpha_{1}\right),
\end{aligned}
$$

where $V_{J}(\mu)$ denotes the $\left(\mu\left(h_{1}\right)+1\right)$-dimensional irreducible representation over $s l(2, \mathbf{C})$. Since $\left(-A_{i+1} \alpha_{0}-B_{i} \alpha_{1}\right)\left(h_{1}\right)=b A_{i+1}-2 B_{i}$, we have

$$
P\left(H^{(J)}\right)=\left\{\left(A_{i+1}, B_{i}+j\right) \mid i \geq 0,0 \leq j \leq b A_{i+1}-2 B_{i}\right\}
$$

and

$$
\operatorname{dim} H_{\left(A_{i+1}, B_{i}+j\right)}^{(J)}=(-1)^{i} .
$$

Hence the denominator identity for the algebra $\mathfrak{g}_{-}^{(J)}$ is equal to

$$
\prod_{\substack{m \in \mathbf{Z}_{>0} \\ n \in \mathbf{Z}_{\geq 0}}}\left(1-p^{m} q^{n}\right)^{\operatorname{dimg} g_{(m, n)}}=1-\sum_{i=0}^{\infty} \sum_{j=0}^{b A_{i+1}-2 B_{i}}(-1)^{i} p^{A_{i+1}} q^{B_{i}+j} .
$$

The set $T^{(J)}(k, l)$ of the partitions of $(k, l)$ into a sum of $\left(A_{i+1}, B_{i}+j\right)$ 's is given by

$$
T^{(J)}(k, l)=\left\{s=\left(s_{i j}\right)_{i, j \geq 0} \mid s_{i j} \in \mathbf{Z}_{\geq 0}, \sum s_{i j}\left(A_{i+1}, B_{i}+j\right)=(k, l)\right\},
$$

and the Witt partition function $W^{(J)}$ is the same as

$$
W^{(J)}(k, l)=\sum_{s \in T^{(J)}(k, l)} \frac{(|s|-1) !}{s !}(-1)^{\sum i s_{i j}} .
$$

Therefore, our root multiplicity formula (3.12) yields: 
Proposition 3.9. Let $\mathfrak{g}=\mathfrak{g}(a, b)$ be the hyperbolic Kac-Moody algebra associated with the generalized Cartan matrix $A=\left(\begin{array}{cc}2 & -a \\ -b & 2\end{array}\right)$ with $a \geq b$ and $a b>4$. Then, for the root $\alpha=-m \alpha_{0}-n \alpha_{1}$, we have

$$
\begin{aligned}
& \operatorname{dim}_{\alpha}=\sum_{d \mid(m, n)} \frac{1}{d} \mu(d) W^{(J)}\left(\frac{m}{d}, \frac{n}{d}\right) \\
& =\sum_{d \mid(m, n)} \frac{1}{d} \mu(d) \sum_{s \in T^{(J)}\left(\frac{m}{d}, \frac{n}{d}\right)} \frac{(|s|-1) !}{s !}(-1)^{\sum i s_{i j}} .
\end{aligned}
$$

Example 3.10. We compute the multiplicity of the root $\alpha=-5 \alpha_{0}-4 \alpha_{1}$ of the algebra $\mathfrak{g}=\mathfrak{g}(4,3)$ which we considered in Example 3.8. The formula (3.31) gives

$$
\operatorname{dimg}(4,3)_{(5,4)}=W^{(J)}(5,4) .
$$

By (3.26), we get

$$
P\left(H^{(J)}\right)=\{(1,0),(1,1), \cdots,(1,3),(5,1),(5,2), \cdots,(5,13),(16,4), \cdots \cdots\},
$$

where

$$
\begin{aligned}
& \operatorname{mult}(1,0)=\cdots=\operatorname{mult}(1,3)=1, \\
& \operatorname{mult}(5,1)=\cdots=\operatorname{mult}(5,13)=-1, \\
& \operatorname{mult}(16,4)=1, \cdots \cdots
\end{aligned}
$$

Since the partitions of $(5,4)$ are given by

$$
\begin{aligned}
& (5,4)=2(1,0)+2(1,1)+(1,2)=3(1,0)+2(1,2) \\
& =3(1,0)+(1,1)+(1,3)=(1,0)+4(1,1),
\end{aligned}
$$

we get

$$
W^{(J)}(5,4)=\frac{4 !}{2 ! 2 !}+\frac{4 !}{3 ! 2 !}+\frac{4 !}{3 !}+\frac{4 !}{4 !}+(-1)=12 .
$$

Hence $\operatorname{dimg}(4,3)_{(5,4)}=12$, as we have seen in Example 3.8.

3.4. The hyperbolic Kac-Moody algebra $\mathfrak{F}$. Let $\mathfrak{F}$ be the hyperbolic KacMoody algebra associated with the generalized Cartan matrix

$$
A=\left(a_{i j}\right)_{i, j=-1,0,1}=\left(\begin{array}{ccc}
2 & -1 & 0 \\
-1 & 2 & -2 \\
0 & -2 & 2
\end{array}\right),
$$

and $I=\{-1,0,1\}$ be the index set for the simple roots of $\mathfrak{F}$. We will apply our root multiplicity formula (3.12) to the algebra $\mathfrak{F}$ and give three different formulas for the root multiplicities of $\mathfrak{F}$.

We first take $J=\{0,1\}$. Then $\mathfrak{F}_{0}^{(J)}=\left\langle e_{0}, e_{1}, f_{0}, f_{1}, h_{0}, h_{1}\right\rangle+\mathfrak{h}$ is isomorphic to the affine Kac-Moody algebra $A_{1}^{(1)}$, and the elements of $W(J)$ can be determined by using Lemma 3.3. For example, we have

$$
W(J)=\left\{r_{-1}, r_{-1} r_{0}, r_{-1} r_{0} r_{1}, r_{-1} r_{0} r_{1} r_{0}, \cdots\right\},
$$

and (3.10) yields

$$
\begin{aligned}
H^{(J)} \cong & V_{J}\left(-\alpha_{-1}\right) \ominus V_{J}\left(-2 \alpha_{-1}-\alpha_{0}\right) \oplus V_{J}\left(-4 \alpha_{-1}-3 \alpha_{0}-\alpha_{1}\right) \\
& \ominus V_{J}\left(-7 \alpha_{-1}-6 \alpha_{0}-3 \alpha_{1}\right) \oplus \cdots
\end{aligned}
$$


where $V_{J}(\lambda)$ denotes the irreducible highest weight module over the affine KacMoody algebra $A_{1}^{(1)}$.

The multiplicity of the weight $\mu$ in $V_{J}(\lambda)$ is given by the number of $\lambda$-paths of weight $\mu$, denoted by $\mathcal{P}_{\lambda, \mu}$, arising from the crystal base theory of quantum affine Lie algebras $[\mathrm{KMN}]$. For a root $\alpha \in \Delta^{-}(J)$, let $P\left(H^{(J)}\right)_{\leq \alpha}=\left\{\tau_{1}, \tau_{2}, \tau_{3}, \cdots\right\}$ be the set of weights of $H^{(J)}$ that can appear as summands in an expression of $\alpha$, and for any $\tau$ dividing $\alpha$, let $T^{(J)}(\tau)$ be the set of all partitions of $\tau$ into a sum of $\tau_{i}$ 's. Then we have

$$
\mathcal{P}_{i} \stackrel{\text { def }}{=} \operatorname{dim} H_{\tau_{i}}^{(J)}=\sum_{\substack{w \in W(J) \\ l(w) \geq 1}}(-1)^{l(w)+1} \mathcal{P}_{w \rho-\rho, \tau_{i}},
$$

and the Witt partition function $W^{(J)}(\tau)$ is given by

$$
W^{(J)}(\tau)=\sum_{s \in T^{(J)}(\tau)} \frac{(|s|-1) !}{s !} \prod \mathcal{P}_{i}^{s_{i}} .
$$

Therefore, our root multiplicity formula (3.12) yields:

Proposition 3.11. Let $\alpha \in \Delta^{-}(J)$ be a root of the hyperbolic Kac-Moody algebra $\mathfrak{F}$. Then we have

$$
\begin{aligned}
& \operatorname{dim} \mathfrak{F}_{\alpha}=\sum_{d \mid \alpha} \frac{1}{d} \mu(d) W^{(J)}\left(\frac{\alpha}{d}\right) \\
& =\sum_{d \mid \alpha} \frac{1}{d} \mu(d) \sum_{s \in T^{(J)}\left(\frac{\alpha}{d}\right)} \frac{(|s|-1) !}{s !} \prod \mathcal{P}_{i}^{s_{i}} .
\end{aligned}
$$

Next, we take $J=J_{1}=\{-1,0\}$. Then $\mathfrak{F}_{0}^{\left(J_{1}\right)}=\left\langle e_{-1}, e_{0}, f_{-1}, f_{0}, h_{-1}, h_{0}\right\rangle+\mathfrak{h} \cong$ $\operatorname{sl}(3, \mathbf{C})+\mathfrak{h}$, and the elements of $W\left(J_{1}\right)$ can be determined by using Lemma 3.3. Hence we have

$$
W\left(J_{1}\right)=\left\{r_{1}, r_{1} r_{0}, r_{1} r_{0} r_{1}, r_{1} r_{0} r_{-1}, \cdots\right\}
$$

and (3.10) yields

$$
\begin{aligned}
H^{\left(J_{1}\right)} \cong & V_{J_{1}}\left(-\alpha_{1}\right) \ominus V_{J_{1}}\left(-\alpha_{0}-3 \alpha_{1}\right) \oplus V_{J_{1}}\left(-3 \alpha_{0}-6 \alpha_{1}\right) \\
& \oplus V_{J_{1}}\left(-\alpha_{-1}-2 \alpha_{0}-5 \alpha_{1}\right) \ominus \cdots
\end{aligned}
$$

where $V_{J_{1}}(\lambda)$ denotes the irreducible highest weight module over $\operatorname{sl}(3, \mathbf{C})$.

The multiplicity of the weight $\mu$ in $V_{J_{1}}(\lambda)$ is given by the number of semistandard tableaux of shape $\lambda$ with weight $\mu$, denoted by $K_{\lambda, \mu}$, which is called the Kostka number (see, for example, [BBL]. For a root $\alpha \in \Delta^{-}\left(J_{1}\right)$, let $P\left(H^{\left(J_{1}\right)}\right)_{\leq \alpha}=$ $\left\{\tau_{1}, \tau_{2}, \tau_{3}, \cdots\right\}$ be the set of weights of $H^{\left(J_{1}\right)}$ that can appear as summands in an expression of $\alpha$, and for any $\tau$ dividing $\alpha$, let $T^{\left(J_{1}\right)}(\tau)$ be the set of all partitions of $\tau$ into a sum of $\tau_{i}$ 's. Then we have

$$
K_{i} \stackrel{\text { def }}{=} \operatorname{dim} H_{\tau_{i}}^{\left(J_{1}\right)}=\sum_{\substack{w \in W\left(J_{1}\right) \\ l(w) \geq 1}}(-1)^{l(w)+1} K_{w \rho-\rho, \tau_{i}},
$$


and the Witt partition function $W^{\left(J_{1}\right)}(\tau)$ is given by

$$
W^{\left(J_{1}\right)}(\tau)=\sum_{s \in T^{\left(J_{1}\right)}(\tau)} \frac{(|s|-1) !}{s !} \prod K_{i}^{s_{i}} .
$$

Therefore, our root multiplicity formula (3.12) yields:

Proposition 3.12. Let $\alpha \in \Delta^{-}\left(J_{1}\right)$ be a root of the hyperbolic Kac-Moody algebra $\mathfrak{F}$. Then we have

$$
\begin{aligned}
& \operatorname{dim} \mathfrak{F}_{\alpha}=\sum_{d \mid \alpha} \frac{1}{d} \mu(d) W^{\left(J_{1}\right)}\left(\frac{\alpha}{d}\right) \\
& =\sum_{d \mid \alpha} \frac{1}{d} \mu(d) \sum_{s \in T^{\left(J_{1}\right)\left(\frac{\alpha}{d}\right)}} \frac{(|s|-1) !}{s !} \prod K_{i}^{s_{i}} .
\end{aligned}
$$

Finally, we take $J=\phi$. Then $\mathfrak{F}_{0}^{(\phi)}=\mathfrak{h}$ and $W(\phi)=W$, the full Weyl group. In $[\mathrm{FF}]$, it was shown that there is an isomorphism $W \cong P G L(2, \mathbf{Z})$ given by

$$
r_{-1} \mapsto \pm\left(\begin{array}{cc}
0 & 1 \\
1 & 0
\end{array}\right), \quad r_{0} \mapsto \pm\left(\begin{array}{cc}
-1 & 1 \\
0 & 1
\end{array}\right), \quad r_{1} \mapsto \pm\left(\begin{array}{cc}
1 & 0 \\
0 & -1
\end{array}\right)
$$

Hence, by (3.10), we have

$$
H^{(\phi)} \cong \sum_{\substack{w \in W \\ l(w) \geq 1}}(-1)^{l(w)+1} \mathbf{C}_{w \rho-\rho}=\sum_{\substack{w \in P G L(2, \mathbf{Z}) \\ w \neq 1}}(-\operatorname{det} w) \mathbf{C}_{w \rho-\rho},
$$

and $P\left(H^{(\phi)}\right)=\{w \rho-\rho \mid w \in P G L(2, \mathbf{Z}), w \neq 1\}$ with $\operatorname{mult}(w \rho-\rho)=-\operatorname{det} w$. Let $\left\{\tau_{i}=w_{i} \rho-\rho \mid i=1,2,3, \cdots\right\}$ be an enumeration of $P\left(H^{(\phi)}\right)$, and denote by $T^{(\phi)}$ the set of all partitions of $\tau$ into a sum of $\tau_{i}$ 's. Then we have

$$
\operatorname{dim} H_{\tau_{i}}^{(\phi)}=-\operatorname{det} w_{i},
$$

and the Witt partition function $W^{(\phi)}$ is given by

$$
W^{(\phi)}(\tau)=\sum_{s \in T^{(\phi)}(\tau)} \frac{(|s|-1) !}{s !} \prod\left(-\operatorname{det} w_{i}\right)^{s_{i}}
$$

Therefore, our root multiplicity formula (3.12) yields:

Proposition 3.13. Let $\alpha \in \Delta^{-}$be a root of the hyperbolic Kac-Moody algebra $\mathfrak{F}$. Then we have

$$
\begin{aligned}
& \operatorname{dim} \mathfrak{F}_{\alpha}=\sum_{d \mid \alpha} \frac{1}{d} \mu(d) W^{(\phi)}\left(\frac{\alpha}{d}\right) \\
& =\sum_{d \mid \alpha} \frac{1}{d} \mu(d) \sum_{s \in T^{(\phi)}\left(\frac{\alpha}{d}\right)} \frac{(|s|-1) !}{s !} \prod\left(-\operatorname{det} w_{i}\right)^{s_{i}} .
\end{aligned}
$$

Remark. (a) For the root $\alpha=-k \alpha_{-1}-l \alpha_{0}-m \alpha_{1}$ with $k, l, m>0$, the above three different expressions of the multiplicity of $\alpha$ will yield a combinatorial identity.

(b) It is still an open problem to prove Frenkel's conjecture on the upper bound of the root multiplicities of $\mathfrak{F}$ :

$$
\operatorname{dim} \mathfrak{F}_{\alpha} \leq p\left(1-\frac{(\alpha \mid \alpha)}{2}\right),
$$

where $p(n)$ denotes the number of partitions of $n$ into a sum of positive integers. 
3.5. The extended hyperbolic Kac-Moody algebras $\mathfrak{F}(a, b)$. In [SUM1], N. Sthanumoorthy and A. Uma Maheswari introduced the notion of extended hyperbolic Kac-Mody algebras. More precisely, let $A=\left(a_{i j}\right)_{i, j \in I}$ be a generalized Cartan matrix and suppose every proper connected subdiagram of the Dynkin diagram of $A$ is of finite, affine, or hyperbolic type. Then the corresponding KacMoody algebra $\mathfrak{g}=\mathfrak{g}(A)$ is called an extended hyperbolic Kac-Moody algebra. For example, consider the generalized Cartan matrix

$$
A=\left(a_{i j}\right)_{i, j=-1,0,1}=\left(\begin{array}{ccc}
2 & -a & 0 \\
-b & 2 & -2 \\
0 & -2 & 2
\end{array}\right) \quad \text { with } a b \geq 4
$$

Then the Kac-Moody algebra $\mathfrak{F}(a, b)$ associated with the matrix $A$ is an extended hyperbolic Kac-Moody algebra.

In [SUM2], the algebra $\mathfrak{F}(a, b)$ was realized as the minimal graded Lie algebra with local part $V \oplus \mathfrak{g}_{0} \oplus V^{*}$, where $\mathfrak{g}_{0}$ is the affine Kac-Moody algebra $A_{1}^{(1)}, V$ is the irreducible highest weight module over $A_{1}^{(1)}$ with highest weight $\lambda$ such that $\lambda\left(h_{0}\right)=b, \lambda\left(h_{1}\right)=0$, and $V^{*}$ is the contragredient module of $V$. When $a=1$, using the homological methods developed in [Ka1] and [Ka2], N. Sthanumoorthy and A. Uma Maheswari gave a multiplicity formula for the roots of level $\leq 3$. In this work, we will apply our root multiplicity formula to the algebra $\mathfrak{F}(a, b)$ and give a multiplicity formula for all the roots of $\mathfrak{F}(a, b)$ in terms of the number of paths arising from the crystal base theory.

Let $I=\{-1,0,1\}$ be the index set for the simple roots of $\mathfrak{F}(a, b)$ and take $J=\{0,1\}$. Then $\mathfrak{F}(a, b)_{0}^{(J)}=\left\langle e_{0}, e_{1}, f_{0}, f_{1}, h_{0}, h_{1}\right\rangle+\mathfrak{h}$ is isomorphic to the affine Kac-Moody algebra $A_{1}^{(1)}$, and the elements of $W(J)$ can be determined by using Lemma 3.3. Thus we have

$$
\begin{aligned}
& W(J)=\left\{r_{-1}, r_{-1} r_{0}, r_{-1} r_{0} r_{-1}, r_{-1} r_{0} r_{1}, r_{-1} r_{0} r_{-1} r_{0}, r_{-1} r_{0} r_{-1} r_{1},\right. \\
&\left.r_{-1} r_{0} r_{-1} r_{0}, \cdots\right\},
\end{aligned}
$$

and (3.10) yields

$$
\begin{aligned}
H^{(J)} \cong & V_{J}\left(-\alpha_{-1}\right) \ominus V_{J}\left(-(a+1) \alpha_{-1}-\alpha_{0}\right) \\
& \oplus V_{J}\left(-a(b+1) \alpha_{-1}-(b+1) \alpha_{0}\right) \\
& \oplus V_{J}\left(-(3 a+1) \alpha_{-1}-3 \alpha_{0}-\alpha_{1}\right) \\
& \ominus V_{J}\left(-a(a b+b-1) \alpha_{-1}-b(a+1) \alpha_{0}\right) \\
& \ominus V_{J}\left(-a(b+3) \alpha_{-1}-(b+3) \alpha_{0}-\alpha_{1}\right) \\
& \ominus V_{J}\left(-(6 a+1) \alpha_{-1}-6 \alpha_{0}-3 \alpha_{1}\right) \oplus \cdots,
\end{aligned}
$$

where $V_{J}(\lambda)$ denotes the irreducible highest weight module over the affine KacMoody algebra $A_{1}^{(1)}$.

The multiplicity of the weight $\mu$ in $V_{J}(\lambda)$ is given by the number of $\lambda$-paths of weight $\mu$, denoted by $\mathcal{P}_{\lambda, \mu}$, as we have seen in the case of the algebra $\mathfrak{F}$. For a root $\alpha \in \Delta^{-}(J)$, let $P\left(H^{(J)}\right)_{\leq \alpha}=\left\{\tau_{1}, \tau_{2}, \tau_{3}, \cdots\right\}$ be the set of weights of $H^{(J)}$ that can appear as summands in an expression of $\alpha$, and for any $\tau$ dividing $\alpha$, let $T^{(J)}(\tau)$ 
be the set of all partitions of $\tau$ into a sum of $\tau_{i}$ 's. Then we have

$$
\mathcal{P}_{i} \stackrel{\text { def }}{=} \operatorname{dim} H_{\tau_{i}}^{(J)}=\sum_{\substack{w \in W(J) \\ l(w) \geq 1}}(-1)^{l(w)+1} \mathcal{P}_{w \rho-\rho, \tau_{i}},
$$

and the Witt partition function $W^{(J)}(\tau)$ is given by

$$
W^{(J)}(\tau)=\sum_{s \in T^{(J)}(\tau)} \frac{(|s|-1) !}{s !} \prod \mathcal{P}_{i}^{s_{i}}
$$

Therefore, our root multiplicity formula (3.12) yields:

Proposition 3.14. Let $\alpha \in \Delta^{-}(J)$ be a root of the extended hyperbolic Kac-Moody algebra $\mathfrak{F}(a, b)$. Then we have

$$
\begin{aligned}
& \operatorname{dim} \mathfrak{F}(a, b)_{\alpha}=\sum_{d \mid \alpha} \frac{1}{d} \mu(d) W^{(J)}\left(\frac{\alpha}{d}\right) \\
& =\sum_{d \mid \alpha} \frac{1}{d} \mu(d) \sum_{s \in T^{(J)}\left(\frac{\alpha}{d}\right)} \frac{(|s|-1) !}{s !} \prod \mathcal{P}_{i}^{s_{i}} .
\end{aligned}
$$

Remark. The above approach can be applied to a more general class of indefinite Kac-Moody algebras that can be realized as the minimal graded Lie algebras with local part $V \oplus \mathfrak{g}_{0} \oplus V^{*}$, where $\mathfrak{g}_{0}$ is a Kac-Moody algebra of classical finite type or classical affine type, $V$ is a direct sum of irreducible highest weight modules over $\mathfrak{g}_{0}$ with dominant integral highest weights, and $V^{*}$ is the contragredient module of $V$ (cf. [BKM1]). For example, one can give a root multiplicity formula for the indefinite Kac-Moody algebra associated with the generalized Cartan matrix

$$
A=\left(\begin{array}{ccc}
2 & -a & 0 \\
-b & 2 & -4 \\
0 & -1 & 2
\end{array}\right)
$$

There have been some efforts to understand the root multiplicities of indefinite KacMoody algebras, including the algebras $\mathfrak{F}$ and $\mathfrak{F}(a, b)$ (see, for example, [BKM1][BKM3], [FF], [Fr], [KMW], [Ka1]-[Ka4], [KM1], [KM2], [SUM2]). However, their general behavior is still mysterious.

\section{Generalized KaC-Moody algebras}

In this section, we will apply our dimension formula (1.13) to generalized KacMoody algebras to derive a closed form root multiplicity formula (Theorem 4.4), which enables us to study the structure of a generalized Kac-Moody algebra $\mathfrak{g}$ as a representation of a Kac-Moody algebra $\mathfrak{g}_{0}$ contained in $\mathfrak{g}$. The choice of $\mathfrak{g}_{0}$ gives rise to various expressions of the root multiplicities of $\mathfrak{g}$, which would yield some interesting combinatorial identities. We will discuss the applications of our root multiplicity formula to various generalized Kac-Moody algebras. In particular, by applying our root multiplicity formula to the Monster Lie algebra, we obtain a recursive relation for the coefficients $c(n)$ of the elliptic modular function:

$$
j(q)-744=\sum_{n=-1}^{\infty} c(n) q^{n}=q^{-1}+196884 q+21493760 q^{2}+\cdots .
$$

(Corollary 4.10. See also [Ka6], [Ju1], [KaK1].) 
4.1. The Weyl-Kac-Borcherds character formula. We first recall some of the basic facts about generalized Kac-Moody algebras ([B2], [Ju2]). Let $I$ be a finite or countably infinite index set. A real matrix $A=\left(a_{i j}\right)_{i, j \in I}$ is called a BorcherdsCartan matrix if (i) $a_{i i}=2$ or $a_{i i} \leq 0$ for all $i \in I$, (ii) $a_{i j} \leq 0$ if $i \neq j$ and $a_{i j} \in \mathbf{Z}$ if $a_{i i}=2$, (iii) $a_{i j}=0$ implies $a_{j i}=0$. We assume that the Borcherds-Cartan matrix $A$ is symmetrizable, i.e., there is a diagonal matrix $D=\operatorname{diag}\left(s_{i} \mid i \in I\right)$ with $s_{i}>0(i \in I)$ such that $D A$ is symmetric. Let $I^{r e}=\left\{i \in I \mid a_{i i}=2\right\}$, $I^{i m}=\left\{i \in I \mid a_{i i} \leq 0\right\}$, and let $\underline{m}=\left(m_{i} \in \mathbf{Z}_{>0} \mid i \in I\right)$ be a collection of positive integers such that $m_{i}=1$ for all $i \in I^{r e}$.

Definition 4.1. The generalized Kac-Moody algebra $\mathfrak{g}=\mathfrak{g}(A, \underline{m})$ associated with a symmetrizable Borcherds-Cartan matrix $A=\left(a_{i j}\right)_{i, j \in I}$ of charge $\underline{m}=\left(m_{i} \mid i \in I\right)$ is the Lie algebra generated by the elements $h_{i}, d_{i}(i \in I), e_{i k}, \overline{f_{i k}}(i \in I, k=$ $\left.1, \cdots, m_{i}\right)$ with the defining relations:

$$
\begin{aligned}
& {\left[h_{i}, h_{j}\right]=\left[d_{i}, d_{j}\right]=\left[h_{i}, d_{j}\right]=0,} \\
& {\left[h_{i}, e_{j l}\right]=a_{i j} e_{j l}, \quad\left[h_{i}, f_{j l}\right]=-a_{i j} f_{j l},} \\
& {\left[d_{i}, e_{j l}\right]=\delta_{i j} e_{j l}, \quad\left[d_{i}, f_{j l}\right]=-\delta_{i j} f_{j l},} \\
& {\left[e_{i k}, f_{j l}\right]=\delta_{i j} \delta_{k l} h_{i},} \\
& \left(a d e_{i k}\right)^{1-a_{i j}}\left(e_{j l}\right)=\left(a d f_{i k}\right)^{1-a_{i j}}\left(f_{j l}\right)=0 \text { if } a_{i i}=2, i \neq j, \\
& {\left[e_{i k}, e_{j l}\right]=\left[f_{i k}, f_{j l}\right]=0 \text { if } a_{i j}=0}
\end{aligned}
$$

$\left(i, j \in I, k=1, \cdots, m_{i}, l=1, \cdots, m_{j}\right)$.

The subalgebra $\mathfrak{h}=\left(\bigoplus_{i \in I} \mathbf{C} h_{i}\right) \oplus\left(\bigoplus_{i \in I} \mathbf{C} d_{i}\right)$ is called the Cartan subalgebra of $\mathfrak{g}$. For each $i \in I$, we define a linear functional $\alpha_{i} \in \mathfrak{h}^{*}$ by

$$
\alpha_{i}\left(h_{j}\right)=a_{j i}, \quad \alpha_{i}\left(d_{j}\right)=\delta_{i j} \quad(i, j \in I) .
$$

The linear functionals $\alpha_{i}(i \in I)$ are called the simple roots of $\mathfrak{g}$.

Let $Q=\bigoplus_{i \in I} \mathbf{Z} \alpha_{i}, Q^{+}=\sum_{i \in I} \mathbf{Z}_{\geq 0} \alpha_{i}$, and $Q^{-}=-Q^{+}$. We define a partial ordering $\geq$ on $\mathfrak{h}^{*}$ by $\lambda \geq \mu$ if and only if $\lambda-\mu \in Q^{+}$. The generalized Kac-Moody algebra $\mathfrak{g}=\mathfrak{g}(A, \underline{m})$ has the root space decomposition

$$
\mathfrak{g}=\bigoplus_{\alpha \in Q} \mathfrak{g}_{\alpha}, \quad \text { where } \mathfrak{g}_{\alpha}=\{x \in \mathfrak{g} \mid[h, x]=\alpha(h) x \text { for all } h \in \mathfrak{h}\} .
$$

An element $\alpha \in Q$ is called a root if $\alpha \neq 0$ and $\mathfrak{g}_{\alpha} \neq 0$, and dimg $\mathfrak{g}_{\alpha}$ is called the multiplicity of the root $\alpha$. A root $\alpha>0$ (resp. $\alpha<0$ ) is called positive (resp. negative). As in the case of Kac-Moody algebras, all the roots are either positive or negative. We denote by $\Delta, \Delta^{+}$, and $\Delta^{-}$the set of all roots, positive roots, and negative roots, respectively. Let $\mathfrak{g}_{+}=\bigoplus_{\alpha>0} \mathfrak{g}_{\alpha}$ and $\mathfrak{g}_{-}=\bigoplus_{\alpha<0} \mathfrak{g}_{\alpha}$. Then we have the triangular decomposition

$$
\mathfrak{g}=\mathfrak{g}_{-} \oplus \mathfrak{g}_{0} \oplus \mathfrak{g}_{+} .
$$

For each $i \in I^{r e}$, we define the simple reflection $r_{i} \in \mathfrak{h}^{*}$ by $r_{i}(\lambda)=\lambda-\lambda\left(h_{i}\right) \alpha_{i}$ $\left(\lambda \in \mathfrak{h}^{*}\right)$. The subgroup $W$ of $G L\left(\mathfrak{h}^{*}\right)$ generated by the $r_{i}$ 's $\left(i \in I^{r e}\right)$ is called the Weyl group of $\mathfrak{g}$. Let $\rho \in \mathfrak{h}^{*}$ be a linear functional satisfying $\rho\left(h_{i}\right)=\frac{1}{2} a_{i i}$ for all $i \in I$. Since the Borcherds-Cartan matrix $A=\left(a_{i j}\right)_{i, j \in I}$ is symmetrizable, there is a $W$-invariant symmetric bilinear form $(\mid)$ on $Q \oplus \mathbf{Z} \rho$ satisfying $\left(\alpha_{i} \mid \alpha_{j}\right)=s_{i} a_{i j}$ and $\left(\rho \mid \alpha_{i}\right)=\frac{1}{2}\left(\alpha_{i} \mid \alpha_{i}\right)$ for all $i, j \in I$. We say that a root $\alpha$ is real if $(\alpha \mid \alpha)>0$, and imaginary if $(\alpha \mid \alpha) \leq 0$. In particular, the simple root $\alpha_{i}$ is real if $a_{i i}=2$, and 
imaginary if $a_{i i} \leq 0$. Note that the imaginary simple roots may have multiplicity $>1$.

As in the case of Kac-Moody algebras, we can define $\mathfrak{h}$-diagonalizable modules, highest weight modules, Verma modules, irreducible highest weight modules, etc. for symmetrizable generalized Kac-Moody algebras.

Let $P^{+}=\left\{\lambda \in \mathfrak{h}^{*} \mid \lambda\left(h_{i}\right) \geq 0\right.$ for all $i \in I, \lambda\left(h_{i}\right) \in \mathbf{Z}_{>0}$ if $\left.a_{i i}=2\right\}$, and let $V(\lambda)$ be the irreducible highest weight module over $\mathfrak{g}$ with highest weight $\lambda$. We denote by $T$ the set of all imaginary simple roots counted with multiplicities, and for $F \subset T$, we write $F \perp \lambda$ if $\lambda\left(h_{i}\right)=0$ for all $\alpha_{i} \in F$. Then the character of $V(\lambda)$ is given by the Weyl-Kac-Borcherds formula:

Proposition 4.2 ([B2], [K3]).

$$
\operatorname{ch} V(\lambda)=\frac{\sum_{\substack{w \in W \\ F \subset T}}(-1)^{l(w)+|F|} e^{w(\lambda+\rho-s(F))-\rho}}{\prod_{\alpha \in \Delta^{-}}\left(1-e^{\alpha}\right)^{\operatorname{dimg}_{\alpha}}},
$$

where $F$ runs over all the finite subsets of $T$ such that any two distinct elements in $F$ are mutually perpendicular, $|F|$ denotes the number of elements in $F$, and $s(F)$ is the sum of elements in $F$.

Letting $\lambda=0$, we obtain the denominator identity for symmetrizable generalized Kac-Moody algebras:

$$
\prod_{\alpha \in \Delta^{-}}\left(1-e^{\alpha}\right)^{\operatorname{dimg}_{\alpha}}=\sum_{\substack{w \in W \\ F \subset T}}(-1)^{l(w)+|F|} e^{w(\rho-s(F))-\rho} .
$$

4.2. The root multiplicity formula. Let $J$ be a finite subset of $I^{r e}$ and we denote by $\Delta_{J}=\Delta \cap\left(\sum_{j \in J} \mathbf{Z} \alpha_{j}\right), \Delta_{J}^{ \pm}=\Delta_{J} \cap \Delta^{ \pm}$, and $\Delta^{ \pm}(J)=\Delta^{ \pm} \backslash \Delta_{J}^{ \pm}$. We also denote by $Q_{J}=Q \cap\left(\sum_{j \in J} \mathbf{Z} \alpha_{j}\right), Q_{J}^{ \pm}=Q_{J} \cap Q^{ \pm}$, and $Q^{ \pm}(J)=Q^{ \pm} \backslash Q_{J}^{ \pm}$. Define $\mathfrak{g}_{0}^{(J)}=\mathfrak{h} \oplus\left(\bigoplus_{\alpha \in \Delta_{J}} \mathfrak{g}_{\alpha}\right)$, and $\mathfrak{g}_{ \pm}^{(J)}=\bigoplus_{\alpha \in \Delta^{ \pm}(J)} \mathfrak{g}_{\alpha}$. Then we have the triangular decomposition:

$$
\mathfrak{g}=\mathfrak{g}_{-}^{(J)} \oplus \mathfrak{g}_{0}^{(J)} \oplus \mathfrak{g}_{+}^{(J)},
$$

where $\mathfrak{g}_{0}^{(J)}$ is the Kac-Moody algebra (with an extended Cartan subalgebra) associated with the generalized Cartan matrix $A_{J}=\left(a_{i j}\right)_{i, j \in J}$, and $\mathfrak{g}_{-}^{(J)}$ (resp. $\mathfrak{g}_{+}^{(J)}$ ) is a direct sum of irreducible highest weight (resp. lowest weight) modules over $\mathfrak{g}_{0}^{(J)}$.

Let $W_{J}=\left\langle r_{j} \mid j \in J\right\rangle$ be the subgroup of $W$ generated by the simple reflections $r_{j}(j \in J)$, and let $W(J)=\left\{w \in W \mid w \Delta^{-} \cap \Delta^{+} \subset \Delta^{+}(J)\right\}$. Then $W_{J}$ is the Weyl group of the Kac-Moody algebra $\mathfrak{g}_{0}^{(J)}$ and $W(J)$ is the set of right coset representatives of $W_{J}$ in $W$. Moreover, the elements in $W(J)$ can be determined by Lemma 3.3 .

As in the case of symmetrizable Kac-Moody algebras, we view $\mathfrak{g}_{-}^{(J)}$ as the $Q_{-}^{(J)}$ graded Lie algebra and apply our dimension formula to the algebra $\mathfrak{g}_{-}^{(J)}$. First of all, we need to determine the homology space

$$
H^{(J)}=\sum_{k=1}^{\infty}(-1)^{k+1} H_{k}\left(\mathfrak{g}_{-}^{(J)}\right)=H_{1}\left(\mathfrak{g}_{-}^{(J)}\right) \ominus H_{2}\left(\mathfrak{g}_{-}^{(J)}\right) \oplus H_{3}\left(\mathfrak{g}_{-}^{(J)}\right) \ominus \cdots .
$$

In $[\mathrm{N}]$, S. Naito proved the Kostant formula for the homology of generalized KacMoody algebras under the assumption that the index set $I$ is finite. In this case, we 
can derive the denominator identity for the algebra $\mathfrak{g}_{-}^{(J)}$ using the Euler-Poincaré principle. However, many interesting generalized Kac-Moody algebras such as the Monster Lie algebra have the Borcherds-Cartan matrix indexed by a countably infinite set. Therefore, even though we believe Naito's method can be generalized to a more general class of generalized Kac-Moody algebras, we will derive the denominator identity for the algebra $\mathfrak{g}_{-}^{(J)}$ directly from the Weyl-Kac-Borcherds character formula.

Proposition 4.3 (cf. [Ka6]). Let $J$ be a finite subset of $I^{\text {re }}$. Then we have

$$
\prod_{\alpha \in Q^{-}(J)}\left(1-e^{\alpha}\right)^{\operatorname{dimg}_{\alpha}}=\sum_{\substack{w \in W(J) \\ F \subset T}}(-1)^{l(w)+|F|} \operatorname{ch} V_{J}(w(\rho-s(F))-\rho),
$$

where $V_{J}(\mu)$ denotes the irreducible highest weight module over the symmetrizable Kac-Moody algebra $\mathfrak{g}_{0}^{(J)}$ with highest weight $\mu$.

Proof. Let $P_{J}^{+} \subset P^{+}$be the set of all $\lambda \in \mathfrak{h}^{*}$ such that $\lambda\left(h_{j}\right) \in \mathbf{Z}_{\geq 0}$ for all $j \in J$. We first show that $w(\rho-s(F))-\rho \in P_{J}^{+}$for all $w \in W(J)$. Since $w(\rho-s(F))-\rho \in Q^{-}$, it is clear that $(w(\rho-s(F))-\rho)\left(h_{j}\right) \in \mathbf{Z}$ for all $j \in J$. Thus it remains to show that $(w(\rho-s(F))-\rho)\left(h_{j}\right) \geq 0$ for all $j \in J$. For each $j \in J$, since $w \in W(J)$, we have $w^{-1}\left(\alpha_{j}\right)>0$. Hence $\left(w \rho \mid \alpha_{j}\right)=\left(\rho \mid w^{-1}\left(\alpha_{j}\right)\right)>0$, which implies $(w \rho)\left(h_{j}\right)=\frac{2\left(w \rho \mid \alpha_{j}\right)}{\left(\alpha_{j} \mid \alpha_{j}\right)}>0$. Therefore, $(w \rho-\rho)\left(h_{j}\right)=(w \rho)\left(h_{j}\right)-1 \geq 0$. Moreover, since $J \subset I^{r e}$, we have $s(F)\left(h_{j}\right) \leq 0$ for all $j \in J$. Hence $(w(\rho-s(F))-\rho)\left(h_{j}\right) \geq 0$ for all $j \in J$.

Now, by the Weyl-Kac character formula, we have

$$
\operatorname{ch} V_{J}(w(\rho-s(F))-\rho)=\frac{\sum_{w^{\prime} \in W_{J}}(-1)^{l\left(w^{\prime}\right)} e^{w^{\prime} w(\rho-s(F))-\rho}}{\prod_{\alpha \in Q_{J}^{-}}\left(1-e^{\alpha}\right)^{\operatorname{dimg} g_{\alpha}}} .
$$

Therefore, the right-hand side of (4.9) yields

$$
\begin{aligned}
& \sum_{\substack{w \in W(J) \\
F \subset T}}(-1)^{l(w)+|F|} \operatorname{ch} V_{J}(w(\rho-s(F))-\rho) \\
= & \sum_{\substack{w \in W(J) \\
F \subset T}}(-1)^{l(w)+|F|} \frac{\sum_{w^{\prime} \in W_{J}}(-1)^{l\left(w^{\prime}\right)} e^{w^{\prime} w(\rho-s(F))-\rho}}{\prod_{\alpha \in Q_{J}^{-}}\left(1-e^{\alpha}\right)^{\operatorname{dim} \mathfrak{g}_{\alpha}}} \\
= & \frac{\sum_{\substack{w \in W(J) \\
w^{\prime} \in W_{J} \\
F \subset T}}(-1)^{l(w)+l\left(w^{\prime}\right)+|F|} e^{w^{\prime} w(\rho-s(F))-\rho}}{\prod_{\alpha \in Q_{J}^{-}}\left(1-e^{\alpha}\right)^{\operatorname{dim} \mathfrak{g}_{\alpha}}} \\
= & \frac{\sum_{\substack{w \in W \\
F \subset T}}(-1)^{l(w)+|F|} e^{w(\rho-s(F))-\rho}}{\prod_{\alpha \in Q_{J}^{-}}\left(1-e^{\alpha}\right)^{\operatorname{dim} \mathfrak{g}_{\alpha}}} .
\end{aligned}
$$

By the denominator identity (4.7), this is equal to

$$
\frac{\prod_{\alpha \in Q^{-}}\left(1-e^{\alpha}\right)^{\operatorname{dimg}_{\alpha}}}{\prod_{\alpha \in Q_{J}^{-}}\left(1-e^{\alpha}\right)^{\operatorname{dimg} g_{\alpha}}}=\prod_{\alpha \in Q^{-}(J)}\left(1-e^{\alpha}\right)^{\operatorname{dimg}_{\alpha}},
$$

which proves the identity (4.9). 
Let

$$
H_{k}^{(J)}=\bigoplus_{\substack{w \in W(J) \\ F \subset T \\ l(w)+|F|=k}} V_{J}(w(\rho-s(F))-\rho)
$$

and define the homology space $H^{(J)}$ of $\mathfrak{g}_{-}^{(J)}$ to be

$$
H^{(J)}=\sum_{k=1}^{\infty}(-1)^{k+1} H_{k}^{(J)}=\sum_{\substack{w \in W(J) \\ F \subset T \\ l(w)+|F| \geq 1}}(-1)^{l(w)+|F|+1} V_{J}(w(\rho-s(F))-\rho)
$$

Let $P\left(H^{(J)}\right)=\left\{\alpha \in Q^{-}(J) \mid \operatorname{dim} H_{\alpha}^{(J)} \neq 0\right\}=\left\{\tau_{1}, \tau_{2}, \tau_{3}, \cdots\right\}$, and $d(i)=$ $\operatorname{dim} H_{\tau_{i}}^{(J)}$ for $i=1,2,3, \cdots$. For $\tau \in Q^{-}(J)$, we denote by $T^{(J)}(\tau)$ the set of all partitions of $\tau$ into a sum of $\tau_{i}$ 's as defined in (1.11), and let $W^{(J)}(\tau)$ be the Witt partition function as defined in (1.12). Then our dimension formula (1.13) yields a closed form root multiplicity formula for all symmetrizable generalized Kac-Moody algebras.

Theorem 4.4 (cf. [Ka6]). Let $\alpha \in \Delta^{-}(J)$ be a root of a symmetrizable generalized Kac-Moody algebra $\mathfrak{g}$. Then we have

$$
\begin{aligned}
& \operatorname{dim}_{\alpha}=\sum_{d \mid \alpha} \frac{1}{d} \mu(d) W^{(J)}\left(\frac{\alpha}{d}\right) \\
& =\sum_{d \mid \alpha} \frac{1}{d} \sum_{s \in T^{(J)}\left(\frac{\alpha}{d}\right)} \frac{(|s|-1) !}{s !} \prod d(i)^{s_{i}} .
\end{aligned}
$$

Suppose the Borcherds-Cartan matrix $A=\left(a_{i j}\right)_{i, j \in I}$ of charge $\underline{m}=\left(m_{i} \in\right.$ $\mathbf{Z}_{>0} \mid i \in I$ ) satisfies: (i) the set $I^{r e}$ is finite, (ii) $a_{i j} \neq 0$ for all $i, j \in I^{i m}$. If we take $J=I^{r e}$, the set of all real indices, then the algebra $\mathfrak{g}_{0}^{(J)}=\mathfrak{h} \oplus\left(\bigoplus_{\alpha \in \Delta_{J}} \mathfrak{g}_{\alpha}\right)$ is the Kac-Moody algebra (with an extended Cartan subalgebra) associated with the generalized Cartan matrix $A_{J}=\left(a_{i j}\right)_{i, j \in J}$, and the generalized Kac-Moody algebra $\mathfrak{g}=\mathfrak{g}_{-}^{(J)} \oplus \mathfrak{g}_{0}^{(J)} \oplus \mathfrak{g}_{+}^{(J)}$ can be realized as the minimal graded Lie algebra with the local part $V \oplus \mathfrak{g}_{0}^{(J)} \oplus V^{*}$, where $V=\bigoplus_{i \in I^{i m}} V_{J}\left(-\alpha_{i}\right)^{\oplus m_{i}}, V^{*}$ is the contragredient module of $V$, and $m_{i}$ 's are the multiplicities of the imaginary simple roots $\alpha_{i}$ 's (cf. [K1], [BKM1], [Ka1], [Ju1]).

By our conditions on the Borcherds-Cartan matrix, the denominator identity of the algebra $\mathfrak{g}_{-}^{(J)}$ is the same as

$$
\begin{aligned}
\prod_{\alpha \in Q^{-}(J)}\left(1-e^{\alpha}\right)^{\operatorname{dimg} g_{\alpha}} & =1-\sum_{\substack{w \in W(J) \\
f \in T \\
l(w)+|F| \geq 1}} \operatorname{ch} V_{J}(w(\rho-s(F))-\rho) \\
& =1-\sum_{i \in I^{i m}} m_{i} \operatorname{ch} V_{J}\left(-\alpha_{i}\right)
\end{aligned}
$$

But, since $\mathfrak{g}=\mathfrak{g}_{-}^{(J)} \oplus \mathfrak{g}_{0}^{(J)} \oplus \mathfrak{g}_{+}^{(J)}$ is the minimal graded Lie algebra with the local part $V \oplus \mathfrak{g}_{0}^{(J)} \oplus V^{*}$, the algebra $\mathfrak{g}_{-}^{(J)}$ is a homomorphic image of the free Lie algebra $\mathcal{F}^{-}=$ $\bigoplus_{\alpha \in Q^{-}(J)} \mathcal{F}_{\alpha}^{-}$generated by $V=\bigoplus_{i \in I^{i m}} V_{J}\left(-\alpha_{i}\right)^{\oplus m_{i}}$. By (2.4), the denominator 
identity of the free Lie algebra $\mathcal{F}^{-}$is equal to

$$
\prod_{\alpha \in Q^{-}(J)}\left(1-e^{\alpha}\right)^{\operatorname{dim} \mathcal{F}_{\alpha}^{-}}=1-\sum_{i \in I^{i m}} m_{i} \operatorname{ch} V_{J}\left(-\alpha_{i}\right) .
$$

In particular, $\operatorname{dim} \mathcal{F}_{\alpha}^{-}=\operatorname{dim}\left(\mathfrak{g}_{-}^{(J)}\right)_{\alpha}$ for all $\alpha \in Q^{-}(J)$. Hence the algebra $\mathfrak{g}_{-}^{(J)}$ is isomorphic to the free Lie algebra generated by $V=\bigoplus_{i \in I^{i m}} V_{J}\left(-\alpha_{i}\right)^{\oplus m_{i}}$. Therefore, we obtain:

Proposition 4.5 (cf. [Ju1], [JW]). Suppose that a Borcherds-Cartan matrix A= $\left(a_{i j}\right)_{i, j \in I}$ of charge $\underline{m}=\left(m_{i} \in \mathbf{Z}_{>0} \mid i \in I\right)$ satisfies: (i) the set $I^{\text {re }}$ is finite, (ii) $a_{i j} \neq 0$ for all $i, j \in I^{i m}$. Let $J=I^{r e}$, and consider the corresponding triangular decomposition of the generalized Kac-Moody algebra:

$$
\mathfrak{g}=\mathfrak{g}(A, \underline{m})=\mathfrak{g}_{-}^{(J)} \oplus \mathfrak{g}_{0}^{(J)} \oplus \mathfrak{g}_{+}^{(J)}
$$

Then the algebra $\mathfrak{g}_{-}^{(J)}=\bigoplus_{\alpha \in \Delta^{-}(J)} \mathfrak{g}_{\alpha}$ (resp. $\mathfrak{g}_{+}^{(J)}=\bigoplus_{\alpha \in \Delta^{+}(J)} \mathfrak{g}_{\alpha}$ ) is isomorphic to the free Lie algebra generated by the space $V=\bigoplus_{i \in I^{i m}} V_{J}\left(-\alpha_{i}\right)^{\oplus m_{i}}$ (resp. $V^{*}=$ $\bigoplus_{i \in I^{i m}} V_{J}^{*}\left(-\alpha_{i}\right)^{\oplus m_{i}}$ ), where $V_{J}(\mu)$ (resp. $V_{J}^{*}(\mu)$ ) denotes the irreducible highest weight (resp. lowest weight) module over the Kac-Moody algebra $\mathfrak{g}_{0}^{(J)}$ with highest weight $\mu$ (resp. lowest weight $-\mu)$.

Remark. Proposition 4.5 shows that, under the above assumptions, the generalized Kac-Moody algebra $\mathfrak{g}=\mathfrak{g}_{-}^{(J)} \oplus \mathfrak{g}_{0}^{(J)} \oplus \mathfrak{g}_{+}^{(J)}$ is isomorphic to the maximal graded Lie algebra with local part $V \oplus \mathfrak{g}_{0}^{(J)} \oplus V^{*}$.

4.3. Low rank cases. In this subsection, we will apply our root multiplicity formula (4.12) to the generalized Kac-Moody algebras associated with rank 1 and rank 2 Borcherds-Cartan matrices, and determine their root multiplicities explicitly. As an illustration of the denominator identity (4.9) and the root multiplicity formula (4.12), we consider the rank 1 case first.

Let $A=(a)$ be a Borcherds-Cartan matrix of charge $\underline{m}=(r)$, where $a=2$ or $a \leq 0, r \in \mathbf{Z}_{>0}$, and let $\mathfrak{g}$ be the generalized Kac-Moody algebra associated with $A=(a)$ and $\underline{m}=(r)$. In this case, we denote by $\alpha$ the only simple root of $\mathfrak{g}$ (which may have multiplicity $>1$ if it is imaginary).

If $a=2$, then we must have $r=1$, and the simple root $\alpha$ is real. Hence the algebra $\mathfrak{g}$ is isomorphic to $\operatorname{sl}(2, \mathbf{C}) \oplus \mathbf{C} d \cong g l(2, \mathbf{C})$.

If $a<0$, then the simple root $\alpha$ is imaginary with multiplicity $r \geq 1$, and hence $T=\{\alpha, \cdots, \alpha\}$ (counted $r$ times). Since there is no real simple root, we should take $J=\phi$ and $W(J)=W=\{1\}$. Consider a subset $F \subset T$ such that any two distinct elements in $F$ are perpendicular. Since $(\alpha \mid \alpha)=a \neq 0, F$ must be either empty or $\{\alpha\}$, which implies

$$
H=H_{1}=\mathbf{C}_{-\alpha} \oplus \cdots \oplus \mathbf{C}_{-\alpha} \quad(r \text { copies }) .
$$

Thus $P(H)=\{-\alpha\}$, $\operatorname{dim} H_{-\alpha}=r$, and the denominator identity for the algebra $\mathfrak{g}_{-}=\bigoplus_{n=1}^{\infty} \mathfrak{g}_{-n \alpha}$ is equal to

$$
\prod_{n=1}^{\infty}\left(1-q^{n}\right)^{\operatorname{dimg}_{-n \alpha}}=1-r q
$$

where $q=e^{-\alpha}$. 
It follows that the algebra $\mathfrak{g}_{-}=\bigoplus_{n=1}^{\infty} \mathfrak{g}_{-n \alpha}$ is isomorphic to the free Lie algebra generated by the homology space $H$, and therefore the Witt formula (2.6) yields

$$
\operatorname{dimg}_{-n \alpha}=\frac{1}{n} \sum_{d \mid n} \mu(d) r^{\frac{n}{d}} .
$$

Indeed, our root multiplicity formula (4.12) gives the same result. Hence the algebra $\mathfrak{g}$ is isomorphic to the maximal graded Lie algebra with local part $H \oplus \mathfrak{h} \oplus H^{*}$. If $r=1$, then the algebra $\mathfrak{g}$ is isomorphic to $g l(2, \mathbf{C})$.

If $a=0$, then the simple root $\alpha$ is imaginary with multiplicity $r \geq 1$, and $T=\{\alpha, \cdots, \alpha\}$ (counted $r$ times) as in the case when $a<0$. Again, we should take $J=\phi$ and $W(J)=W=\{1\}$. But, since $(\alpha \mid \alpha)=0$, we can take $F$ to be any subset of $T$. Therefore, by (4.10), we obtain

$$
H_{k}= \begin{cases}\mathbf{C}_{-k \alpha} \oplus \cdots \oplus \mathbf{C}_{-k \alpha} & \text { for } k \leq r\left(\left(\begin{array}{l}
r \\
k
\end{array}\right) \text { copies }\right), \\
0 & \text { for } k>r,\end{cases}
$$

and $P(H)=\{-\alpha,-2 \alpha, \cdots,-r \alpha\}$ with $\operatorname{dim} H_{-k \alpha}=(-1)^{k+1}\left(\begin{array}{l}r \\ k\end{array}\right)$. Hence the denominator identity for the algebra $\mathfrak{g}_{-}=\bigoplus_{n=1}^{\infty} \mathfrak{g}_{-n \alpha}$ is the same as

$$
\prod_{n=1}^{\infty}\left(1-q^{n}\right)^{\operatorname{dimg}_{-n \alpha}}=1-\sum_{k=1}^{r}(-1)^{k+1}\left(\begin{array}{l}
r \\
k
\end{array}\right) q^{k}=(1-q)^{r}
$$

which implies

$$
\operatorname{dimg}_{-n \alpha}= \begin{cases}r & \text { if } n=1, \\ 0 & \text { if } n \geq 2 .\end{cases}
$$

Hence the algebra $\mathfrak{g}$ is isomorphic to the $(2 r+1)$-dimensional Heisenberg Lie algebra plus one-dimensional derivations.

On the other hand, we have

$$
T(-k \alpha)=\left\{s=\left(s_{1}, \cdots, s_{r}\right) \mid s_{i} \in \mathbf{Z}_{\geq 0}, s_{1}+2 s_{2}+\cdots+r s_{r}=k\right\}=T_{r}(k)
$$

which is the set of all partitions of $k$ with parts $\leq r$, and the Witt partition function $W(-k \alpha)$ is equal to

$$
\begin{aligned}
W(-k \alpha) & =\sum_{s \in T_{r}(k)} \frac{(|s|-1) !}{s !} \prod\left[(-1)^{i+1}\left(\begin{array}{l}
r \\
i
\end{array}\right)\right]^{s_{i}} \\
& =(-1)^{k} \sum_{s \in T_{r}(k)} \frac{(|s|-1) !}{s !}(-1)^{|s|} \prod\left(\begin{array}{l}
r \\
i
\end{array}\right)^{s_{i}} .
\end{aligned}
$$

Therefore, by our root multiplicity formula (4.12), we obtain

$$
\begin{aligned}
& \operatorname{dim}_{-n \alpha}=\sum_{d \mid n} \frac{1}{d} \mu(d) W\left(-\frac{n \alpha}{d}\right) \\
& =\sum_{d \mid n} \frac{1}{d} \mu(d)(-1)^{\frac{n}{d}} \sum_{s \in T_{r}\left(\frac{n}{d}\right)} \frac{(|s|-1) !}{s !}(-1)^{|s|} \prod\left(\begin{array}{l}
r \\
i
\end{array}\right)^{s_{i}} .
\end{aligned}
$$

Combining (4.22) with (4.19) yields a combinatorial identity

$$
\sum_{d \mid n} \frac{1}{d} \mu(d)(-1)^{\frac{n}{d}} \sum_{s \in T_{r}\left(\frac{n}{d}\right)} \frac{(|s|-1) !}{s !}(-1)^{|s|} \prod\left(\begin{array}{l}
r \\
i
\end{array}\right)^{s_{i}}=0 \text { for } n \geq 2 .
$$


Now we consider the generalized Kac-Moody algebra $\mathfrak{g}=\mathfrak{g}(A, \underline{m})$ associated with the Borcherds-Cartan matrix $A=\left(\begin{array}{cc}2 & -a \\ -b & -c\end{array}\right)$ of charge $\underline{m}=(1, r)$ with $a, b, c, r \in \mathbf{Z}_{>0}$. Let $I=\{0,1\}$ be the index set for the simple roots of $\mathfrak{g}$. Then $\alpha_{0}$ is the real simple root and $\alpha_{1}$ is the imaginary simple root with multiplicity $r \geq 1$. Thus we have $T=\left\{\alpha_{1}, \cdots, \alpha_{1}\right\}$ (counted $r$ times), and since $\left(\alpha_{1} \mid \alpha_{1}\right)<0, F$ can be either empty or $\left\{\alpha_{1}\right\}$.

If we take $J=\phi$, then $\mathfrak{g}_{0}^{(J)}=\mathfrak{h}$, the Cartan subalgebra, and $W(\phi)=W=$ $\left\{1, r_{0}\right\}$. Hence by $(4.10)$ we obtain

$$
H^{(\phi)}=\mathbf{C}_{-\alpha_{0}} \oplus \mathbf{C}_{-\alpha_{1}}^{\oplus r} \ominus \mathbf{C}_{-(a+1) \alpha_{0}-\alpha_{1}}^{\oplus r} .
$$

By identifying $-k \alpha_{0}-l \alpha_{1} \in Q^{-}$with $(k, l) \in \mathbf{Z}_{\geq 0} \times \mathbf{Z}_{\geq 0}$, we have

$$
P^{(\phi)}=\{(1,0),(0,1),(a+1,1)\},
$$

and

$$
\operatorname{dim} H_{(1,0)}^{(\phi)}=1, \quad \operatorname{dim} H_{(0,1)}^{(\phi)}=r, \quad \operatorname{dim} H_{(a+1,1)}^{(\phi)}=-r .
$$

Therefore, the denominator identity for the algebra $\mathfrak{g}_{-}^{(\phi)}$ is equal to

$$
\prod_{\substack{m, n \in \mathbf{Z}_{\geq 0} \\(m, n) \neq(0,0)}}\left(1-p^{m} q^{n}\right)^{\operatorname{dim} g_{(m, n)}}=1-p-r q\left(1-p^{a+1}\right),
$$

where $p=e^{-\alpha_{0}}$ and $q=e^{-\alpha_{1}}$.

Moreover, the set $T^{(\phi)}(k, l)$ is given by

$$
\begin{aligned}
T^{(\phi)}(k, l)= & \left\{s=(\alpha, \beta, \gamma) \mid \alpha, \beta, \gamma \in \mathbf{Z}_{\geq 0},\right. \\
& \alpha(1,0)+\beta(0,1)+\gamma(a+1,1)=(k, l)\} \\
= & \left\{(k-(a+1) t, l-t, t) \mid t=0,1, \cdots, \min \left(\left[\frac{k}{a+1}\right], l\right)\right\} .
\end{aligned}
$$

Hence the Witt partition function $W^{(\phi)}(k, l)$ is equal to

$$
W^{(\phi)}(k, l)=r^{l} \sum_{t=0}^{\min \left(\left[\frac{k}{a+1}\right], l\right)} \frac{(k+l-(a+1) t-1) !}{(k-(a+1) t) !(l-t) ! t !}(-1)^{t},
$$

and our root multiplicity formula (4.12) yields:

Proposition 4.6. Let $\mathfrak{g}=\mathfrak{g}(A, \underline{m})$ be the generalized Kac-Moody algebra associated with the Borcherds-Cartan matrix $A=\left(\begin{array}{cc}2 & -a \\ -b & -c\end{array}\right)$ of charge $\underline{m}=(1, r)$ with $a, b, c, r \in \mathbf{Z}_{>0}$.

Then, for the root $\alpha=-m \alpha_{0}-n \alpha_{1}$, we have

$$
\begin{aligned}
& \operatorname{dimg}_{\alpha}=\sum_{d \mid(m, n)} \frac{1}{d} \mu(d) W^{(\phi)}\left(\frac{m}{d}, \frac{n}{d}\right) \\
& =\sum_{d \mid(m, n)} \frac{1}{d} \mu(d) r^{\frac{n}{d}} \sum_{t=0}^{\min \left(\left[\frac{m}{d(a+1)}\right], \frac{n}{d}\right)} \frac{\left(\frac{m}{d}+\frac{n}{d}-(a+1) t-1\right) !}{\left(\frac{m}{d}-(a+1) t\right) !\left(\frac{n}{d}-t\right) ! t !}(-1)^{t} .
\end{aligned}
$$


Now, if we take $J=\{0\}$, then $\mathfrak{g}_{0}^{(J)}=\left\langle e_{0}, f_{0}, h_{0}\right\rangle+\mathfrak{h} \cong \operatorname{sl}(2, \mathbf{C})+\mathfrak{h}$, and $W(J)=\{1\}$. By (4.10), we obtain

$$
H^{(J)}=V_{J}\left(-\alpha_{1}\right) \oplus \cdots \oplus V_{J}\left(-\alpha_{1}\right) \quad(r \text { copies }),
$$

where $V_{J}\left(-\alpha_{1}\right)$ is the $(a+1)$-dimensional irreducible representation of $\operatorname{sl}(2, \mathbf{C})$. It follows that $P\left(H^{(J)}\right)=\{(i, 1) \mid i=0,1, \cdots, a\}$ with $\operatorname{dim} H_{(i, 1)}^{(J)}=r$ for all $i=$ $0,1, \cdots, a$. Therefore, the denominator identity for the algebra $\mathfrak{g}_{-}^{(J)}$ is equal to

$$
\prod_{\substack{m \in \mathbf{Z}_{\geq 0} \\ n \in \mathbf{Z}_{>0}}}\left(1-p^{m} q^{n}\right)^{\operatorname{dimg} g_{(m, n)}}=1-\frac{r q\left(1-p^{a+1}\right)}{1-p} .
$$

For $k \in \mathbf{Z}_{\geq 0}$ and $l \in \mathbf{Z}_{>0}$, consider the set

$$
\begin{aligned}
& T^{(J)}(k, l)=\left\{s=\left(s_{0}, s_{1}, \cdots, s_{a}\right) \mid s_{i} \in \mathbf{Z}_{\geq 0},\right. \\
& \left.s_{0}(0,1)+s_{1}(1,1)+\cdots+s_{a}(a, 1)=(k, l)\right\} \\
& =\left\{s=\left(s_{0}, s_{1}, \cdots, s_{a}\right) \mid s_{i} \in \mathbf{Z}_{\geq 0}\right. \text {, } \\
& \left.s_{0}+s_{1}+\cdots+s_{a}=l, s_{1}+2 s_{2}+\cdots+a s_{a}=k\right\} .
\end{aligned}
$$

If $k=0$, then $s_{0}=l, s_{1}=\cdots=s_{a}=0$, which implies $W^{(J)}(0, l)=\frac{1}{l} r^{l}$. It follows that

$$
\operatorname{dimg}_{-n \alpha_{1}}=\frac{1}{n} \sum_{d \mid n} \mu(d) r^{\frac{n}{d}} .
$$

Therefore, the subalgebra $\mathcal{F}_{0}=\bigoplus_{n=1}^{\infty} \mathfrak{g}_{-n \alpha_{1}}$ is isomorphic to the free Lie algebra generated by the space $\mathbf{C}_{-\alpha_{1}} \oplus \cdots \oplus \mathbf{C}_{-\alpha_{1}}$ ( $r$ copies).

If $k, l>0$, note that each partition $s \in T^{(J)}(k, l)$ can be written as $s=\left(s_{0}, \varphi\right)$, where $s_{0}$ runs from 0 to $l-1$ and $\varphi=\left(s_{1}, \cdots, s_{a}\right)$ corresponds to a partition of $k$ with parts $\leq a$ of length $l-s_{0}$. Thus we have

$$
\begin{aligned}
T^{(J)}(k, l)= & \left\{s=\left(s_{0}, \varphi\right) \mid s_{0}=0,1, \cdots, l-1,\right. \\
& \left.\varphi \in T_{a}(k) \text { with }|\varphi|=l-s_{0}\right\} \\
= & \left\{s=(l-|\varphi|, \varphi)\left|\varphi \in T_{a}(k),\right| \varphi \mid=1,2, \cdots, l\right\},
\end{aligned}
$$

and the Witt partition function $W^{(J)}(k, l)$ is given by

$$
W^{(J)}(k, l)=r^{l} \sum_{\substack{\varphi \in T_{a}(k) \\|\varphi| \leq l}} \frac{(l-1) !}{(l-|\varphi|) ! \varphi !} .
$$

Therefore, our root multiplicity formula (4.12) yields:

Proposition 4.7. Let $\mathfrak{g}=\mathfrak{g}(A, \underline{m})$ be the generalized Kac-Moody algebra associated with the Borcherds-Cartan matrix $A=\left(\begin{array}{cc}2 & -a \\ -b & -c\end{array}\right)$ of charge $\underline{m}=(1, r)$ with $a, b, c, r \in \mathbf{Z}_{>0}$. 
Then, for the root $\alpha=-m \alpha_{0}-n \alpha_{1}$ with $m, n>0$, we have

$$
\begin{aligned}
& \operatorname{dimg}_{\alpha}=\sum_{d \mid(m, n)} \frac{1}{d} \mu(d) W^{(J)}\left(\frac{m}{d}, \frac{n}{d}\right) \\
& =\sum_{d \mid(m, n)} \frac{1}{d} \mu(d) r^{\frac{n}{d}} \sum_{\substack{\varphi \in T_{a}\left(\frac{m}{d}\right) \\
|\varphi| \leq \frac{n}{d}}} \frac{\left(\frac{n}{d}-1\right) !}{\left(\frac{n}{d}-|\varphi|\right) ! \varphi !} .
\end{aligned}
$$

Hence the algebra $\mathfrak{g}$ is isomorphic to the maximal graded Lie algebra with local part $H^{(J)} \oplus(s l(2, \mathbf{C})+\mathfrak{h}) \oplus H^{(J) *}$.

By comparing the formulas (4.28) and (4.34), we can show by induction that $W^{(\phi)}(k, l)=W^{(J)}(k, l)$ for all $k, l>0$. Consequently, we obtain the following combinatorial identity:

Proposition 4.8 (cf. [KaK2]).

$$
\sum_{t=0}^{\min \left(\left[\frac{k}{a+1}\right], l\right)} \frac{(k+l-(a+1) t-1) !}{(k-(a+1) t) !(l-t) ! t !}(-1)^{t}=\sum_{\substack{\varphi \in T_{a}(k) \\|\varphi| \leq l}} \frac{(l-1) !}{(l-|\varphi|) ! \varphi !} .
$$

4.4. Monstrous Lie algebras. The classification theorem of finite simple groups tells that there are exactly 26 sporadic simple groups besides the family of alternating groups on $n$ letters $(n \geq 5)$ and the families of simple groups of Lie type (see, for example, [GLS]). The largest among the sporadic simple groups has order

$$
2^{46} \cdot 3^{20} \cdot 5^{9} \cdot 7^{6} \cdot 11^{2} \cdot 13^{3} \cdot 17 \cdot 19 \cdot 23 \cdot 29 \cdot 31 \cdot 41 \cdot 47 \cdot 59 \cdot 71,
$$

and it is called the Monster due to its enormous size.

The trivial character degree of the Monster simple group $G$ is, by definition, one, and the smallest nontrivial irreducible character degree of $G$ is 196883 [FLT]. It was noticed by McKay that $1+196883=196884$, which is the first nontrivial coefficient of the elliptic modular function

$$
j(q)-744=\sum_{n \geq-1} c(n) q^{n}=q^{-1}+196884 q+21493760 q^{2}+\cdots \cdots .
$$

Later, Thompson found that the first few coefficients of the modular function $j(q)-744$ are simple linear combinations of the irreducible character degrees of $G$ $[\mathrm{T}]$. Motivated by these observations, Conway and Norton conjectured that there exists an infinite dimensional graded representation $V^{\natural}=\bigoplus_{n \geq-1} V_{n}^{\natural}$ of the Monster simple group $G$ with $\operatorname{dim} V_{n}^{\natural}=c(n)$ such that the Thompson series

$$
T_{g}(q)=\sum_{n \geq-1} \operatorname{Tr}\left(g \mid V_{n}^{\natural}\right) q^{n}=\sum_{n \geq-1} c_{g}(n) q^{n}
$$

are the normalized generators of the genus zero function fields arising from certain discrete subgroups of $P S L(2, \mathbf{R})[\mathrm{CN}]$. Their conjecture is referred to as the Moonshine conjecture.

The natural graded representation $V^{\natural}=\bigoplus_{n \geq-1} V_{n}^{\natural}$ of the Monster simple group $G$ in the Moonshine conjecture, called the Moonshine module, was constructed by Frenkel, Lepowsky, and Meurman using the theory of vertex (operator) algebras $[\mathrm{FLM}]$. They also calculated the Thompson series for some conjugacy classes of the Monster, and verified the Moonshine conjecture for these Thompson series. 
In [B5], Borcherds completed the proof of the Moonshine conjecture by constructing a $I I_{1,1}$-graded Lie algebra $L=\bigoplus_{(m, n) \in I I_{1,1}} L_{(m, n)}$, called the Monster Lie algebra, where $I I_{1,1}$ is the 2-dimensional even Lorentzian lattice associated with the matrix $\left(\begin{array}{cc}0 & -1 \\ -1 & 0\end{array}\right)$. We will briefly recall Borcherds' construction of the Monster Lie algebra given in [B5].

A vertex algebra $V$ over $\mathbf{C}$ is a complex vector space with an infinite family of bilinear products, written $u_{n} v$ for $u, v \in V, n \in \mathbf{Z}$, such that

(i) $u_{n} v=0$ for $n \gg 0$,

(ii) for all $u, v, w \in V$ and $m, n, q \in \mathbf{Z}$, we have

$$
\begin{aligned}
& \sum_{i \in \mathbf{Z}}\left(\begin{array}{c}
m \\
i
\end{array}\right)\left(u_{q+i} v\right)_{m+n-i} w \\
& =\sum_{i \in \mathbf{Z}}(-1)^{i}\left(\begin{array}{c}
q \\
i
\end{array}\right)\left(u_{m+q-i}\left(v_{n+i} w\right)-(-1)^{q} v_{n+q-i}\left(u_{m+i} w\right)\right),
\end{aligned}
$$

(iii) there is an element $\mathbf{1} \in V$ such that $v_{n} \mathbf{1}=0$ if $n \geq 0$ and $v_{-1} \mathbf{1}=v$ for all $v \in V$.

For example, let $L$ be an even lattice and $\widehat{L}$ be its central extension by a group of order 2. Then we can construct a vertex algebra $V_{L}=S\left(\bigoplus_{i>0} L_{i}\right) \otimes \mathbf{C}[\widehat{L}]$, where $\mathbf{C}[\widehat{L}]$ is a group ring of the double covering $\widehat{L}$ of $L$ and $S\left(\bigoplus_{i>0} L_{i}\right)$ is the ring of polynomials over the sum of an infinite number of copies of $L_{i}=L \otimes_{\mathbf{z}} \mathbf{C}$ [B1]. Another interesting example is the Moonshine module $V^{\natural}=\bigoplus_{n \geq-1} V_{n}^{\natural}$ constructed in $[\mathrm{FLM}]$.

We define the operator $D$ of a vertex algebra $V$ by $D(v)=v_{-2} \mathbf{1}$. The vector space $V / D V$ is a Lie algebra with the bracket defined by $[\bar{u}, \bar{v}]=\overline{u_{0} v}$, where denotes the corresponding equivalence class in $V / D V$ [B1]. A conformal vector of central charge $c$ of a vertex algebra $V$ is defined to be an element $\omega$ in $V$ such that (i) $\omega_{0} v=D(v)$ for all $v \in V$, (ii) $\omega_{1} \omega=2 \omega, \omega_{3} \omega=c / 2, \omega_{i} \omega=0$ for $i=2$ or $i>3$, and (iii) any element of $V$ is a sum of eigenvectors of the operator $L_{0}=\omega_{1}$ with integral eigenvalues. For a conformal vector $\omega$ of $V$, we define the operator $L_{i}$ on $V$ by $L_{i}=\omega_{i+1}$. Then these operators satisfy the relations:

$$
\left[L_{i}, L_{j}\right]=(i-j) L_{i+j}+\frac{1}{12} \delta_{i+j, 0}\left(i^{3}-i\right) c,
$$

and hence $V$ becomes a module over the Virasoro algebra. The operator $L_{-1}$ is equal to $D$. We define the subspace $P^{i}=\left\{v \in V \mid L_{0}(v)=i v, L_{i}(v)=0\right.$ for $\left.i>0\right\}$. The space $P^{1} /\left(D V \cap P^{1}\right)$ is a Lie subalgebra of $V / D V$, which is equal to $P^{1} / D P^{0}$ for the vertex algebra $V_{L}$ or for the Moonshine module $V^{\natural}$. It is shown that the Lie algebra $P^{1} / D P^{0}$ is a generalized Kac-Moody algebra [B2], [B4].

Let $V^{\natural}$ be the Moonshine module, and let $V_{I I_{1,1}}$ be the vertex algebra associated with the lattice $I I_{1,1}$. Then the tensor product $V^{\natural} \otimes V_{I I_{1,1}}$ is also a vertex algebra, and the space $P^{1} / D P^{0}$ for this vertex algebra is a Lie algebra with a symmetric invariant bilinear form ( , ). We define the Monster Lie algebra $L$ to be the quotient of the Lie algebra $P^{1} / D P^{0}$ by the kernel of the form $($,$) . Hence the Monster Lie$ algebra $L$ is a $I I_{1,1}$-graded representation of the Monster simple group $G$ such that $L_{(m, n)} \cong V_{m n}^{\natural}$ as $G$-modules for $(m, n) \neq(0,0)$. In particular, we have

$$
\operatorname{dim} L_{(m, n)}=\operatorname{dim} V_{m n}^{\natural}=c(m n) \text { for all }(m, n) \neq(0,0) .
$$


On the other hand, the Monster Lie algebra can be regarded as a generalized KacMoody algebra [B5]. We take $I=\{-1\} \cup\{1,2,3, \cdots\}$ as the index set, and consider the Borcherds-Cartan matrix $A=(-(i+j))_{i, j \in I}$ of charge $\underline{m}=(c(i) \mid i \in I)$, where $c(i)$ are the coefficients of the elliptic modular function $j(q)-744=\sum_{n=-1}^{\infty} c(n) q^{n}$. Let $\mathfrak{g}=\mathfrak{g}(A, \underline{m})$ be the generalized Kac-Moody algebra associated with the data $A=(-(i+j))_{i, j \in I}$ and $\underline{m}=(c(i) \mid i \in I)$. Then $\alpha_{-1}$ is the only real simple root, and $\alpha_{i}$ are the imaginary simple roots with multiplicity $c(i)(i \geq 1)$. Thus $W=\left\{1, r_{-1}\right\}$ and $T=\left\{\alpha_{i}, \cdots, \alpha_{i} \mid i \geq 1\right\}$, where each $\alpha_{i}$ is counted $c(i)$ times. Since $\left(\alpha_{i} \mid \alpha_{i}\right)=-2 i \neq 0$ for $i \geq 1, F$ can be either empty or $\left\{\alpha_{i}\right\}$ (c(i) choices). Hence, if we take $J=\phi$, we obtain by (4.10)

$$
H^{(\phi)}=\mathbf{C}_{-\alpha_{-1}} \oplus\left(\bigoplus_{i=1}^{\infty} \mathbf{C}_{-\alpha_{i}}^{\oplus c(i)}\right) \ominus\left(\bigoplus_{i=1}^{\infty} \mathbf{C}_{-i \alpha_{-1}-\alpha_{i}}^{\oplus c(i)}\right) .
$$

Therefore, the denominator identity for the algebra $\mathfrak{g}_{-}^{(\phi)}$ is equal to

$$
\prod_{\alpha \in Q^{-}}\left(1-e^{\alpha}\right)^{\operatorname{dimg}_{\alpha}}=1-e^{-\alpha_{-1}}-\sum_{i=1}^{\infty} c(i) e^{-\alpha_{i}}+\sum_{i=1}^{\infty} c(i) e^{-i \alpha_{-1}-\alpha_{i}} .
$$

We identify the simple roots $\alpha_{-1}$ with $(1,-1)$ and $\alpha_{i}$ with $(1, i)(i \geq 1)$. Then the generalized Kac-Moody algebra $\mathfrak{g}=\mathfrak{g}(A, \underline{m})$ becomes a $I I_{1,1}$-graded Lie algebra, and the denominator identity for the algebra $\mathfrak{g}_{-}^{(\phi)}$ can be written as

$$
\begin{aligned}
\prod_{\substack{m>0 \\
n \in \mathbf{Z}}}\left(1-p^{m} q^{n}\right)^{\operatorname{dimg} g_{(m, n)}} & =1-p q^{-1}-\sum_{i=1}^{\infty} c(i) p q^{i}+\sum_{i=1}^{\infty} c(i) p^{i+1} \\
& =p\left(p^{-1}+\sum_{i=1}^{\infty} c(i) p^{i}\right)-p\left(q^{-1}+\sum_{i=1}^{\infty} c(i) q^{i}\right) \\
& =p(j(p)-j(q)),
\end{aligned}
$$

where $p=e^{-(1,0)}$ and $q=e^{-(0,1)}$.

In [B5], Borcherds proved the following product identity for the elliptic modular function $j$ :

$$
p^{-1} \prod_{\substack{m>0 \\ n \in \mathbf{Z}}}\left(1-p^{m} q^{n}\right)^{c(m n)}=j(p)-j(q) .
$$

Therefore, we obtain

$$
\operatorname{dim} \mathfrak{g}_{(m, n)}=c(m n) \text { for all } m>0, n \in \mathbf{Z},
$$

and hence the Monster Lie algebra $L=\bigoplus_{(m, n) \in I I_{1,1}} L_{(m, n)}$ is isomorphic to the $I I_{1,1}$-graded generalized Kac-Moody algebra associated with the Borcherds-Cartan matrix $A=(-(i+j))_{i, j \in I}$ of charge $\underline{m}=(c(i) \mid i \in I)$ (with a modified Cartan subalgebra).

We will apply our root multiplicity formula (4.12) to the Monster Lie algebra $L=$ $\bigoplus_{(m, n) \in I I_{1,1}} L_{(m, n)}$. Take $J=\{-1\}$. Then $L_{0}^{(J)}=\left\langle e_{-1}, f_{-1}, h_{-1}\right\rangle \cong s l(2, \mathbf{C})+\mathfrak{h}$, and $W(J)=\{1\}$. By (4.10), we obtain

$$
H^{(J)}=H_{1}^{(J)}=\bigoplus_{i=1}^{\infty} V_{J}\left(-\alpha_{i}\right)^{\oplus c(i)}
$$


where $V_{J}\left(-\alpha_{i}\right)$ is the $i$-dimensional irreducible representation of $\operatorname{sl}(2, \mathbf{C})$ (since $\left.-\alpha_{i}\left(h_{-1}\right)=i-1\right)$. It follows that

$$
P\left(H^{(J)}\right)=\left\{(-i,-j) \mid i, j \in \mathbf{Z}_{>0}\right\} \quad \text { with } \operatorname{dim} H_{(-i,-j)}^{(J)}=c(i+j-1) .
$$

Therefore, the denominator identity for the algebra $L_{-}^{(J)}$ is equal to

$$
\prod_{m, n=1}^{\infty}\left(1-p^{m} q^{n}\right)^{\operatorname{dim} L_{(m, n)}}=1-\sum_{i, j=1}^{\infty} c(i+j-1) p^{i} q^{j} .
$$

For $k, l>0$, we have

$$
T^{(J)}(k, l)=T(k, l)=\left\{s=\left(s_{i j}\right)_{i, j \geq 1} \mid s_{i j} \in \mathbf{Z}_{\geq 0}, \sum s_{i j}(i, j)=(k, l)\right\},
$$

the set of all partitions of $(k, l)$ into a sum of ordered pairs of positive integers, and the Witt partition function $W^{(J)}(k, l)$ is given by

$$
W^{(J)}(k, l)=\sum_{s \in T(k, l)} \frac{(|s|-1) !}{s !} \prod c(i+j-1)^{s_{i j}} .
$$

Therefore, our root multiplicity formula (4.12) yields:

Proposition 4.9. Let $L=\bigoplus_{(m, n) \in I I_{1,1}} L_{(m, n)}$ be the Monster Lie algebra. Then, for $m, n \in \mathbf{Z}_{>0}$, we have

$$
\operatorname{dim} L_{(m, n)}=\sum_{d \mid(m, n)} \frac{1}{d} \mu(d) \sum_{s \in T\left(\frac{m}{d}, \frac{n}{d}\right)} \frac{(|s|-1) !}{s !} \prod c(i+j-1)^{s_{i j}} .
$$

Combined with (4.37), we obtain the following interesting relations for the coefficients $c(n)$ of the elliptic modular function $j(q)-744=\sum_{n=-1}^{\infty} c(n) q^{n}$ :

Corollary 4.10 (cf. [Ka6], [Ju1], [KaK1]).

$$
c(m n)=\sum_{d \mid(m, n)} \frac{1}{d} \mu(d) \sum_{s \in T\left(\frac{m}{d}, \frac{n}{d}\right)} \frac{(|s|-1) !}{s !} \prod c(i+j-1)^{s_{i j}} .
$$

Remark. In [JLW] and [KK], the relation (4.47) was generalized to the relation of the coefficients $c_{g}(n)$ of the Thompson series

$$
T_{g}(q)=\sum_{n \geq-1} \operatorname{Tr}\left(g \mid V_{n}^{\natural}\right) q^{n}=\sum_{n \geq-1} c_{g}(n) q^{n} .
$$

More precisely, for $m, n>0$, we have

$$
c_{g}(m n)=\sum_{d \mid(m, n)} \frac{1}{d} \mu(d) \sum_{s \in T\left(\frac{m}{d}, \frac{n}{d}\right)} \frac{(|s|-1) !}{s !} \prod c_{g^{d}}(i+j-1)^{s_{i j}} .
$$

It was pointed out in $[\mathrm{JLW}]$ that these relations completely determine all the coefficients $c_{g}(n)$ if the values of $c_{h}(1), c_{h}(2), c_{h}(3)$, and $c_{h}(5)$ are known for all $h \in G$. In particular, the relation (4.47) is a complete recursive relation determining the coefficients $c(n)$ of the elliptic modular function $j(q)-744$.

More generally, let $A=(-(i+j))_{i, j \in I}$ be the Borcherds-Cartan matrix of the Monster Lie algebra $(I=\{-1\} \cup\{1,2,3, \cdots\})$ and let $f(q)=\sum_{n=-1}^{\infty} f(n) q^{n}$ be a formal power series where $f(-1)=1, f(0)=0$, and $f(n) \in \mathbf{Z}_{\geq 0}$ for all $n \geq$ 1. Consider the generalized Kac-Moody algebra $\mathfrak{g}=\mathfrak{g}(A, f)$ associated with the

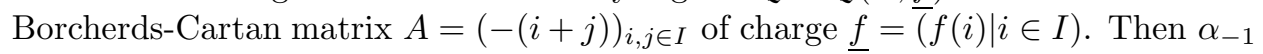


is the only real simple root and $\alpha_{i}$ are the imaginary simple roots with multiplicity $f(i)(i \geq 1)$. (We just neglect those $\alpha_{i}$ 's for which $f(i)=0$.) By identifying the simple roots $\alpha_{-1}$ with $(1,-1)$ and $\alpha_{i}$ with $(1, i)(i \geq 1)$, the generalized KacMoody algebra $\mathfrak{g}=\mathfrak{g}(A, f)$ becomes a $I I_{1,1}$-graded Lie algebra. This $I I_{1,1}$-graded generalized Kac-Moody algebra will be called the Monstrous Lie algebra associated with the formal power series $f(q)=\sum_{n=-1}^{\infty} f(n) q^{n}$, and we will denote it by $L(f)=$ $\bigoplus_{(m, n) \in I I_{1,1}} L(f)_{(m, n)}$.

For example, the Monstrous Lie algebra associated with the elliptic modular function $j(q)-744$ is the Monster Lie algebra, and the Monstrous Lie algebras associated with the Thompson series $T_{g}(q)=\sum_{n=-1}^{\infty} c_{g}(n) q^{n}$ with $c_{g}(n) \geq 0$ for all $n \geq 1$ are the Monstrous Lie algebras considered in [B5]. Of course, we can generalize this notion to define the Monstrous Lie superalgebras associated with any formal power series $f(q)=\sum_{n=-1}^{\infty} f(n) q^{n}$, where $f(-1)=1, f(0)=0$, and $f(n) \in \mathbf{Z}$ for all $n \in \mathbf{Z}$ (see [Ka8]), which generalizes the notion of Monstrous Lie superalgebras considered in [B5].

Let

$$
L(f)_{+}=\bigoplus_{\substack{m>0 \\ n \in \mathbf{Z}}} L(f)_{(m, n)} \text { and } L(f)_{-}=\bigoplus_{\substack{m>0 \\ n \in \mathbf{Z}}} L(f)_{(-m, n)} .
$$

By the same argument for the Monster Lie algebra, the denominator identity for the algebra $L(f)_{-}$is the same as

$$
\prod_{\substack{m>0 \\ n \in \mathbf{Z}}}\left(1-p^{m} q^{n}\right)^{\operatorname{dim} L(f)_{(m, n)}}=p(f(p)-f(q)),
$$

where $p=e^{-(1,0)}$ and $q=e^{-(0,1)}$.

Moreover, if we take $J=\{-1\}$, then, as we have seen for the Monster Lie algebra, we have $L(f)_{0}^{(J)} \cong \operatorname{sl}(2, \mathbf{C})+\mathfrak{h}$,

$$
H^{(J)}=H_{1}^{(J)}=\bigoplus_{i=1}^{\infty} V_{J}\left(-\alpha_{i}\right)^{\oplus f(i)}
$$

and

$$
P\left(H^{(J)}\right)=\left\{(-i,-j) \mid i, j \in \mathbf{Z}_{>0}\right\} \text { with } \operatorname{dim} H_{(-i,-j)}^{(J)}=f(i+j-1) .
$$

Consequently, the denominator identity for the algebra $L(f)_{-}^{(J)}$ is equal to

$$
\prod_{m, n=1}^{\infty}\left(1-p^{m} q^{n}\right)^{\operatorname{dim} L(f)_{(m, n)}}=1-\sum_{i, j=1}^{\infty} f(i+j-1) p^{i} q^{j}
$$

and our root multiplicity formula (4.12) yields:

Proposition 4.11. Let $L(f)=\bigoplus_{(m, n) \in I I_{1,1}} L(f)_{(m, n)}$ be the Monstrous Lie algebra associated with a formal power series $f(q)=\sum_{n=-1}^{\infty} f(n) q^{n}$, where $f(-1)=1$, $f(0)=0$, and $f(n) \in \mathbf{Z}_{\geq 0}$ for all $n \geq 1$.

Then, for $m, n \in \mathbf{Z}_{>0}$, we have

$$
\operatorname{dim} L(f)_{(m, n)}=\sum_{d \mid(m, n)} \frac{1}{d} \mu(d) \sum_{s \in T\left(\frac{m}{d}, \frac{n}{d}\right)} \frac{(|s|-1) !}{s !} \prod f(i+j-1)^{s_{i j}},
$$

where $T(k, l)$ denotes the set of all partitions of $(k, l)$ into a sum of ordered pairs of positive integers. 
Remark. It follows from Proposition 4.5 or Proposition 4.11 that the algebra $L(f)_{-}^{(J)}$ is isomorphic to the free Lie algebra generated by

$$
H^{(J)}=\bigoplus_{i=1}^{\infty} V_{J}\left(-\alpha_{i}\right)^{\oplus f(i)}
$$

where $H^{(J)}$ is regarded as a $\left(\mathbf{Z}_{>0} \times \mathbf{Z}_{>0}\right)$-graded vector space

$$
H^{(J)}=\bigoplus_{i, j=1}^{\infty} H_{(-i,-j)}^{(J)} \text { with } \operatorname{dim} H_{(-i,-j)}^{(J)}=f(i+j-1)
$$

Hence the algebra $L(f)$ is isomorphic to the maximal graded Lie algebra with local part $H^{(J)} \oplus(\operatorname{sl}(2, \mathbf{C})+\mathfrak{h}) \oplus H^{(J) *}$.

Example 4.12. (a) If $f(q)=q^{-1}$, then $L(f) \cong \operatorname{sl}(2, \mathbf{C}) \oplus \mathbf{C} d \cong g l(2, \mathbf{C})$.

(b) If $f(q)=q^{-1}+r q$, then $P\left(H^{(J)}\right)=\{(-1,-1)\}$ with $\operatorname{dim} H_{(-1,-1)}^{(J)}=r$. Hence the denominator identity for the algebra $L(f)_{-}^{(J)}$ is equal to

$$
\prod_{m, n=1}^{\infty}\left(1-p^{m} q^{n}\right)^{\operatorname{dim} L(f)_{(m, n)}}=1-r p q
$$

Moreover, we have

$$
W^{(J)}(k, k)=\frac{1}{k} r^{k}, \text { and } W^{(J)}(k, l)=0 \text { if } k \neq l,
$$

which yields

$$
\operatorname{dim} L(f)_{(m, n)}= \begin{cases}\frac{1}{n} \sum_{d \mid n} \mu(d) r^{\frac{n}{d}} & \text { if } m=n \\ 0 & \text { if } m \neq n .\end{cases}
$$

Therefore, $L(f)_{-}=\bigoplus_{n=1}^{\infty} L(f)_{(-n,-n)}$ is isomorphic to the free Lie algebra generated by an $r$-dimensional vector space (see Corollary 2.2).

(c) If $f(q)=q^{-1}+r q+r q^{2}+r q^{3}+\cdots$, then $\operatorname{dim} H_{(-i,-j)}^{(J)}=r$ for all $i, j \in \mathbf{Z}_{>0}$, and the denominator identity for the algebra $L(f)_{-}^{(J)}$ is the same as

$$
\prod_{m, n=1}^{\infty}\left(1-p^{m} q^{n}\right)^{\operatorname{dim} L(f)_{(m, n)}}=1-\sum_{i, j=1}^{\infty} r p^{i} q^{j}=1-\frac{r p q}{(1-p)(1-q)} .
$$

Hence we have

$$
W^{(J)}(k, l)=\sum_{s \in T(k, l)} \frac{(|s|-1) !}{s !} r^{|s|}
$$

and

$$
\operatorname{dim} L(f)_{(m, n)}=\sum_{d \mid(m, n)} \frac{1}{d} \mu(d) \sum_{s \in T\left(\frac{m}{d}, \frac{n}{d}\right)} \frac{(|s|-1) !}{s !} r^{|s|}
$$

Therefore, the algebra $L(f)_{-}^{(J)}$ is isomorphic to the free Lie algebra generated by the $\left(\mathbf{Z}_{>0} \times \mathbf{Z}_{>0}\right)$-graded vector space $V=\bigoplus_{i, j=1}^{\infty} V_{(i, j)}$ with $\operatorname{dim} V_{(i, j)}=r$ for all $i, j \geq 1$ (see Proposition 2.10). 
(d) If $f(q)=T_{g}(q)=\sum_{n=-1}^{\infty} c_{g}(n) q^{n}$, the Thompson series, then the denominator identity for the algebra $L(f)_{-}^{(J)}$ is equal to

$$
\prod_{m, n=1}^{\infty}\left(1-p^{m} q^{n}\right)^{\operatorname{dim} L(f)_{(m, n)}}=1-\sum_{i, j=1}^{\infty} c_{g}(i+j-1) p^{i} q^{j},
$$

and, for $m, n \in \mathbf{Z}_{>0}$, we have

$$
\operatorname{dim} L(f)_{(m, n)}=\sum_{d \mid(m, n)} \frac{1}{d} \mu(d) \sum_{s \in T\left(\frac{m}{d}, \frac{n}{d}\right)} \frac{(|s|-1) !}{s !} \prod c_{g}(i+j-1)^{s_{i j}} .
$$

When $m$ and $n$ are relatively prime, the identity (4.48) yields

$$
\operatorname{dim} L(f)_{(m, n)}=\sum_{s \in T(m, n)} \frac{(|s|-1) !}{s !} \prod c_{g}(i+j-1)^{s_{i j}}=c_{g}(m n) .
$$

It would be an interesting task to characterize all the (genus 0 ) modular functions $f(q)=\sum_{n=-1}^{\infty} f(n) q^{n}$ satisfying the identity

$$
f(m n)=\sum_{s \in T(m, n)} \frac{(|s|-1) !}{s !} \prod f(i+j-1)^{s_{i j}}
$$

when $m$ and $n$ are relatively prime (cf. [KK]). We expect our approach can shed some light on this problem.

\section{Product identities And GRAded LiE Algebras}

In this section, we will discuss the relation of the graded Lie algebras and the product identities for normalized formal power series. Let $\Gamma$ be a countable abelian semigroup satisfying the finiteness condition given in Section 1. Consider a normalized formal power series

$$
1-\sum_{\alpha \in \Gamma} d(\alpha) e^{\alpha} \text { with } d(\alpha) \in \mathbf{Z} \text { for all } \alpha \in \Gamma .
$$

Suppose we have a product identity for the above formal power series:

$$
\prod_{\alpha \in \Gamma}\left(1-e^{\alpha}\right)^{A(\alpha)}=1-\sum_{\alpha \in \Gamma} d(\alpha) e^{\alpha}
$$

with $A(\alpha) \in \mathbf{Z}_{\geq 0}$ for all $\alpha \in \Gamma$. We would like to construct a $\Gamma$-graded Lie algebra $L=\bigoplus_{\alpha \in \Gamma} L_{\alpha}$ whose denominator identity is the same as (5.2), which would imply

$$
\operatorname{dim} L_{\alpha}=A(\alpha) \text { for all } \alpha \in \Gamma .
$$

This problem is equivalent to constructing a $\Gamma$-graded Lie algebra $L=\bigoplus_{\alpha \in \Gamma} L_{\alpha}$ such that the character of the homology space $H=\sum_{k=1}^{\infty}(-1)^{k+1} H_{k}(L)$ is given by $\operatorname{ch} H=\sum_{\alpha \in \Gamma} d(\alpha) e^{\alpha}$.

Once we have constructed such a $\Gamma$-graded Lie algebra $L$, let $P(H)=$ $\{\alpha \in \Gamma \mid d(\alpha) \neq 0\}=\left\{\tau_{1}, \tau_{2}, \tau_{3}, \cdots\right\}$, and let $d(i)=d\left(\tau_{i}\right)$ for $i=1,2,3, \cdots$. Then, by applying our dimension formula (1.13) to the $\Gamma$-graded Lie algebra $L$, we would obtain a combinatorial identity:

$$
A(\alpha)=\sum_{d \mid \alpha} \frac{1}{d} \mu(d) \sum_{s \in T\left(\frac{\alpha}{d}\right)} \frac{(|s|-1) !}{s !} \prod d(i)^{s_{i}},
$$


where $T(\tau)$ denotes the set of all partitions of $\tau$ into a sum of $\tau_{i}$ 's as defined in (1.11).

Example 5.1. (a) Consider the binomial expansion

$$
(1-q)^{r}=\sum_{k=0}^{r}(-1)^{k}\left(\begin{array}{l}
r \\
k
\end{array}\right) q^{k} .
$$

Let $L=\mathbf{C} x_{1} \oplus \cdots \oplus \mathbf{C} x_{r}$ be the $r$-dimensional abelian Lie algebra with basis $\left\{x_{1}, \cdots, x_{r}\right\}$. Then we have

$$
H_{k}(L)=\Lambda^{k}(L)=\operatorname{Span}\left\{x_{i_{1}} \wedge \cdots \wedge x_{i_{k}} \mid 1 \leq i_{1}<\cdots<i_{k} \leq r\right\} .
$$

Hence, by the Euler-Poincaré principle, the identity (5.5) can be interpreted as the denominator identity for the $r$-dimensional abelian algebra $L$, and by (5.4), we recover the combinatorial identity (4.23).

(b) In [K2], Jacobi's triple product identity

$$
\prod_{n=1}^{\infty}\left(1-p^{n} q^{n}\right)\left(1-p^{n-1} q^{n}\right)\left(1-p^{n} q^{n-1}\right)=\sum_{k \in \mathbf{Z}}(-1)^{k} p^{\frac{k(k-1)}{2}} q^{\frac{k(k+1)}{2}}
$$

was interpreted as the denominator identity for the affine Kac-Moody algebra of type $A_{1}^{(1)}$. In fact, Kac showed that all the Macdonald identities [M] are equivalent to the denominator identities for affine Kac-Moody algebras [K2]. $[\mathrm{S} 2])$ :

(c) Recall the definition of the Ramanujan tau-function $\tau(k)$ (see, for example,

$$
\begin{aligned}
\Delta(q) & =q \prod_{n=1}^{\infty}\left(1-q^{n}\right)^{24}=\sum_{k=1}^{\infty} \tau(k) q^{k} \\
& =q-24 q^{2}+252 q^{3}-1472 q^{4}-\cdots
\end{aligned}
$$

We can rewrite it as

$$
\prod_{n=1}^{\infty}\left(1-q^{n}\right)^{24}=1-\sum_{k=1}^{\infty}(-\tau(k+1)) q^{k}
$$

If we can construct a $\mathbf{Z}_{>0}$-graded Lie algebra $L=\bigoplus_{n=1}^{\infty} L_{n}$ such that $\operatorname{dim} H_{k}(L)=$ $-\tau(k+1)$, then (5.8) would be the denominator identity for this Lie algebra $L$ and we would have $\operatorname{dim} L_{n}=24$ for all $n \geq 1$. But it is more natural to interpret (5.8) as the denominator identity for the free Lie superalgebra generated by the $\mathbf{Z}_{>0}$-graded vector space $V=\bigoplus_{i=1}^{\infty} V_{i}$ with superdimensions $\operatorname{Dim} V_{i}=-\tau(i+1)$ for $i \geq 1$ (see $[\mathrm{Ka} 8])$.

In general, it is quite difficult and complicated to construct a graded Lie algebra corresponding to the product identity (5.2). However, if $d(\alpha) \in \mathbf{Z}_{\geq 0}$ for all $\alpha \in \Gamma$, any product identity of the form (5.2) can always be interpreted as the denominator identity for some suitably defined free Lie algebras. More precisely, let $V=\bigoplus_{\alpha \in \Gamma} V_{\alpha}$ be a $\Gamma$-graded vector space over $\mathbf{C}$ with $\operatorname{dim} V_{\alpha}=d(\alpha)$ for all $\alpha \in \Gamma$. Then the right-hand side of (5.2) can be interpreted as $1-\operatorname{ch} V$. Let $L$ be the free Lie algebra generated by $V$. As we have seen in Section 2, the free Lie algebra $L$ has a $\Gamma$-gradation $L=\bigoplus_{\alpha \in \Gamma} L_{\alpha}$ induced by that of $V$, and the denominator identity (2.4) for $L$ is the same as the product identity (5.2). In particular, we have $\operatorname{dim} L_{\alpha}=A(\alpha)$ for all $\alpha \in \Gamma$, which yields the combinatorial identity (5.4). 
Example 5.2. Recall the product identity (4.40) for the elliptic modular function $j$ proved in [B5]:

$$
p^{-1} \prod_{\substack{m>0 \\ n \in \mathbf{Z}}}\left(1-p^{m} q^{n}\right)^{c(m n)}=j(p)-j(q) .
$$

Observing that $c(0)=0$ and $c(-k)=0$ for $k>1$, it can be written as

$$
\begin{aligned}
\prod_{m, n=1}^{\infty}\left(1-p^{m} q^{n}\right)^{c(m n)} & =\frac{j(p)-j(q)}{p^{-1}-q^{-1}} \\
& =1-\sum_{i, j=1}^{\infty} c(i+j-1) p^{i} q^{j} .
\end{aligned}
$$

Let $V=\bigoplus_{i, j=1}^{\infty} V_{(i, j)}$ be a $\left(\mathbf{Z}_{>0} \times \mathbf{Z}_{>0}\right)$-graded vector space over $\mathbf{C}$ with $\operatorname{dim} V_{(i, j)}=$ $c(i+j-1)$, and let $L=\bigoplus_{m, n=1}^{\infty} L_{(m, n)}$ be the free Lie algebra generated by $V$. Then the product identity (5.9) for the elliptic modular function $j$ is the denominator identity for the free Lie algebra $L$, and we have $\operatorname{dim} L_{(m, n)}=c(m n)$. Hence we obtain a simple proof of the recursive relation (4.47) using the free Lie algebra $L$ (cf. $[\mathrm{KaK} 1])$.

In [B7], Borcherds gives a very important method for constructing automorphic forms on $O_{s+2,2}(\mathbf{R})^{+}$with infinite product expansions. One of the main results in $[\mathrm{B} 7]$ is the following:

Theorem 5.3 ([B7]). Let $I I_{s+2,2}$ be the even unimodular lattice with signature $s$. Suppose that $f(\tau)=\sum_{n \in \mathbf{Z}} c(n) q^{n}$ is a meromorphic modular form of weight $-\frac{s}{2}$ for $S L(2, \mathbf{Z})$ with integral coefficients, with poles only at cusps, and with $24 \mid c(0)$ if $s=0$. Then there exists a unique vector $\rho \in I I_{s+2,2}$ such that

$$
\Phi(v)=e^{-2 \pi i(\rho, v)} \prod_{r>0}\left(1-e^{-2 \pi i(r, v)}\right)^{c\left(-\frac{(r, r)}{2}\right)}
$$

is a meromorphic automorphic form of weight $\frac{c(0)}{2}$ for $O_{I I_{s+2,2}}(\mathbf{Z})^{+}$.

If $s=2$ and $f(q)=j(q)-744$, we recover the product identity (4.40), which is the denominator identity for the Monster Lie algebra [B7]. If $s=24$ and $f(q)=\frac{1}{\Delta(q)}$, then we obtain

$$
e^{\rho} \prod_{r>0}\left(1-e^{r}\right)^{p_{24}\left(1-\frac{(r, r)}{2}\right)}=\sum_{\substack{w \in W \\ n \in \mathbf{Z}_{>0}}}(\operatorname{det} w) \tau(n) e^{w(n \rho)},
$$

where $\tau(n)$ is the Ramanujan tau-function, $p_{24}(n)$ denotes the number of partitions of $n$ into parts of 24 colors, and $W$ is the reflection group of the 26-dimensional even unimodular Lorentzian lattice $I I_{25,1}$. The identity (5.11) is the denominator identity for the fake Monster Lie algebra [B3], [B7].

Unfortunately, most of the automorphic forms with infinite products that are produced by Theorem 5.3 cannot be interpreted as the denominator identities for generalized Kac-Moody algebras, for most of them involve vectors of norm at least 4 in the lattice, which cannot be a positive root of any generalized Kac-Moody algebra [B8]. Still, it seems possible to construct graded Lie algebras and Lie superalgebras whose denominator identities are the modular products given by Theorem 5.3.

Another very important result in [B7] is: 
Theorem $5.4([\mathrm{~B} 7])$. Consider

$$
H(\tau)=\sum_{n \in \mathbf{Z}} H(n) q^{n}=-\frac{1}{12}+\frac{1}{3} q^{3}+\frac{1}{2} q^{4}+q^{7}+q^{8} \cdots,
$$

where $H(n)$ is the Hurwitz class number for the discriminant $-n$ if $n>0$ and $H(0)=-\frac{1}{12}$. Suppose that $f_{0}(\tau)=\sum_{n \in \mathbf{Z}} c_{0}(n) q^{n}$ is a meromorphic modular form of weight $\frac{1}{2}$ for $\Gamma_{0}(4)$ with integral coefficients, with poles only at cusps, and with $c_{0}(n)=0$ if $n \equiv 2,3(\bmod 4)$. Put

$$
\Psi(\tau)=q^{-h} \prod_{n>0}\left(1-q^{n}\right)^{c_{0}\left(n^{2}\right)},
$$

where $h$ is the constant term of $f_{0}(\tau) H(\tau)$. Then $\Psi$ is a meromorphic modular form for some characters of $S L(2, \mathbf{Z})$ of integral weight with leading coefficient 1 , all of whose zeros and poles are either at cusps or imaginary quadratic irrationals.

For example, if $f_{0}(\tau)=12 \theta(\tau)=12 \sum_{n \in \mathbf{Z}} q^{n^{2}}$, then we get $\Psi(\tau)=\Delta(q)=$ $q \prod_{n=1}^{\infty}\left(1-q^{n}\right)^{24}[\mathrm{~B} 7]$. Moreover, let

$$
\begin{gathered}
F(\tau)=\sum_{n \in \mathbf{Z}_{>0}, \text { odd }} \sigma_{1}(n) q^{n}=q+4 q^{3}+6 q^{5}+\cdots, \\
\theta(\tau)=\sum_{n \in \mathbf{Z}} q^{n^{2}}=1+2 q+2 q^{4}+\cdots
\end{gathered}
$$

and define

$$
\begin{aligned}
f_{0}(\tau)= & F(\tau) \theta(\tau) \frac{\left(\theta(\tau)^{4}-2 F(\tau)\right)\left(\theta(\tau)^{4}-16 F(\tau)\right) E_{6}(4 \tau)}{\Delta(4 \tau)}+56 \theta(\tau) \\
= & \sum_{n \in \mathbf{Z}} c_{0}(n) q^{n}=q^{-3}-248 q+26752 q^{4}-\cdots, \\
g_{0}(\tau) & =(j(4 \tau)-876) \theta(\tau) \\
& -2 F(\tau) \theta(\tau) \frac{\left(\theta(\tau)^{4}-2 F(\tau)\right)\left(\theta(\tau)^{4}-16 F(\tau)\right) E_{6}(4 \tau)}{\Delta(4 \tau)} \\
& =\sum_{n \in \mathbf{Z}} b_{0}(n) q^{n}=q^{-4}+6+504 q+143388 q^{4}-\cdots .
\end{aligned}
$$

Then Theorem 5.4 yields the product identities for the elliptic modular function $j$ and the Eisenstein series $E_{4}, E_{6}, E_{8}, E_{10}$, and $E_{14}$ [B7]:

$$
\begin{gathered}
j(\tau)=q^{-1}+744+196884 q+\cdots=q^{-1} \prod_{n=1}^{\infty}\left(1-q^{n}\right)^{3 c_{0}\left(n^{2}\right)}, \\
E_{4}(\tau)=1+240 \sum_{n=1}^{\infty} \sigma_{3}(n) q^{n}=\prod_{n=1}^{\infty}\left(1-q^{n}\right)^{c_{0}\left(n^{2}\right)+8} \\
E_{6}(\tau)=1-504 \sum_{n=1}^{\infty} \sigma_{5}(n) q^{n}=\prod_{n=1}^{\infty}\left(1-q^{n}\right)^{b_{0}\left(n^{2}\right)}
\end{gathered}
$$




$$
\begin{gathered}
E_{8}(\tau)=1+480 \sum_{n=1}^{\infty} \sigma_{7}(n) q^{n}=\prod_{n=1}^{\infty}\left(1-q^{n}\right)^{2 c_{0}\left(n^{2}\right)+16}, \\
E_{10}(\tau)=1-264 \sum_{n=1}^{\infty} \sigma_{9}(n) q^{n}=\prod_{n=1}^{\infty}\left(1-q^{n}\right)^{b_{0}\left(n^{2}\right)+c_{0}\left(n^{2}\right)+8} \\
E_{14}(\tau)=1-24 \sum_{n=1}^{\infty} \sigma_{13}(n) q^{n}=\prod_{n=1}^{\infty}\left(1-q^{n}\right)^{b_{0}\left(n^{2}\right)+2 c_{0}\left(n^{2}\right)+16} .
\end{gathered}
$$

The product identities for the Eisenstein series $E_{6}(\tau), E_{10}(\tau)$, and $E_{14}(\tau)$ can be interpreted as the denominator identities for the free Lie algebras $L=\bigoplus_{n=1}^{\infty} L_{n}$ generated by the $\mathbf{Z}_{>0}$-graded vector spaces $V=\bigoplus_{n=1}^{\infty} V_{n}$ with $\operatorname{dim} V_{n}=504 \sigma_{5}(n)$, $264 \sigma_{9}(n)$, and $24 \sigma_{13}(n)$, respectively. Therefore, the dimensions of the homogeneous subspaces $L_{n}$ are given by

$$
\begin{gathered}
\operatorname{dim} L_{n}=b_{0}\left(n^{2}\right) \quad\left(\text { corresponding to } E_{6}(\tau)\right), \\
\operatorname{dim} L_{n}=b_{0}\left(n^{2}\right)+c_{0}\left(n^{2}\right)+8 \quad\left(\text { corresponding to } E_{10}(\tau)\right), \\
\operatorname{dim} L_{n}=b_{0}\left(n^{2}\right)+2 c_{0}\left(n^{2}\right)+16 \quad\left(\text { corresponding to } E_{14}(\tau)\right),
\end{gathered}
$$

and (5.4) yields the following combinatorial identities:

$$
\begin{gathered}
b_{0}\left(n^{2}\right)=\sum_{d \mid n} \frac{1}{d} \mu(d) \sum_{s \in T\left(\frac{n}{d}\right)} \frac{(|s|-1) !}{s !} 504^{|s|} \prod \sigma_{5}(i)^{s_{i}}, \\
b_{0}\left(n^{2}\right)+c_{0}\left(n^{2}\right)+8=\sum_{d \mid n} \frac{1}{d} \mu(d) \sum_{s \in T\left(\frac{n}{d}\right)} \frac{(|s|-1) !}{s !} 264^{|s|} \prod \sigma_{9}(i)^{s_{i}}, \\
b_{0}\left(n^{2}\right)+2 c_{0}\left(n^{2}\right)+16=\sum_{d \mid n} \frac{1}{d} \mu(d) \sum_{s \in T\left(\frac{n}{d}\right)} \frac{(|s|-1) !}{s !} 24^{|s|} \prod \sigma_{13}(i)^{s_{i}} .
\end{gathered}
$$

On the other hand, these product identities can be interpreted as the denominator identities for the rank 1 generalized Kac-Moody algebras with only one imaginary simple root with multipicity $504 \sigma_{5}(n), 264 \sigma_{9}(n)$, and $24 \sigma_{13}(n)$, respectively [B7]. The rest of the product identities listed above (and many others) can be interpreted as the denominator identities for some free Lie superalgebras. In [Ka8], we will give a more comprehensive discussion on the relation of the graded Lie superalgebras and the product identities for the normalized formal power series.

\section{REFERENCES}

[BBL] Benkart, G. M., Britten, D. J., Lemire, F. W., Stability in modules for classical Lie algebras - a constructive approach, Mem. Amer. Math. Soc. 430 (1990). MR 90m:17012

[BKM1] Benkart, G. M., Kang, S.-J., Misra, K. C., Graded Lie algebras of Kac-Moody type, Adv. Math. 97 (1993), 154-190. MR 94b:17039

[BKM2] Benkart, G. M., Kang, S.-J., Misra, K. C., Indefinite Kac-Moody algebras of classical type, Adv. Math. 105 (1994), 76-110. MR 95d:17023

[BKM3] Benkart, G. M., Kang, S.-J., Misra, K. C., Indefinite Kac-Moody algebras of special linear type, Pacific J. Math. 170 (1995), 379-404. MR 96k:17021

[BM] Berman, S., Moody, R. V., Multiplicities in Lie algebras, Proc. Amer. Math. Soc. 76 (1979), 223-228. MR 80h:17013 
[B1] Borcherds, R. E., Vertex algebras, Kac-Moody algebras and the monster, Proc. Natl. Acad. Sci. USA 83 (1986), 3068-3071. MR 87m:17033

[B2] Borcherds, R. E., Generalized Kac-Moody algebras, J. Algebra 115 (1988), 501-512. MR 89g:17004

[B3] Borcherds, R. E., The Monster Lie algebra, Adv. Math. 83 (1990), 30-47. MR 91k:17027

[B4] Borcherds, R. E., Central extensions of generalized Kac-Moody algebras, J. Algebra 140 (1991), 330-335. MR 92g:17031

[B5] Borcherds, R. E., Monstrous moonshine and monstrous Lie superalgebras, Invent. Math. 109 (1992), 405-444. MR 94f: 11030

[B6] Borcherds, R. E., A characterization of generalized Kac-Moody algebras, J. Algebra 174 (1995), 1073-1079. MR 96e:17058

[B7] Borcherds, R. E., Automorphic forms on $\mathrm{O}_{s+2,2}(\mathbf{R})$ and infinite products, Invent. Math. 120 (1995), 161-213. MR 96j:11067

[B8] Borcherds, R. E., Automorphic forms and Lie algebras, in Current Developments in Mathematics, International Press (1996), 1-27.

[Bo] Bourbaki, N., Lie Groups and Lie Algebras, Part 1, Hermann, Paris, 1975.

[Cas] Cassels, J. W. S., Rational Quadratic Forms, Academic Press, 1978. MR 80m:10019

[CE] Cartan, H., Eilenberg, S., Homological Algebra, Princeton University Press, 1956. MR 17:1040e

[CN] Conway, J. H., Norton, S., Monstrous moonshine, Bull. Lond. Math. Soc. 11 (1979), 308-339. MR 81j:20028

[F] Feingold, A. J., A hyperbolic GCM algebra and the Fibonacci numbers, Proc. Amer. Math. Soc. 80 (1980), 379-385. MR 81k:17009

[FF] Feingold, A. J., Frenkel, I. B., A hyperbolic Kac-Moody algebra and the theory of Siegel modular forms of genus 2, Math. Ann. 263 (1983), 87-144. MR 86a:17006

[FLT] Fischer, B., Livingstone, D., Thorne, M. P., The characters of the "Monster" simple group, Birmingham, 1978.

[Fr] Frenkel, I. B., Representations of Kac-Moody algebras and dual resonance models, in Applications of Group Theory in Physics and Mathematical Physics, Lectures in Applied Math. 21 (1985), 325-353. MR 87b:17010

[FLM] Frenkel, I. B., Lepowsky, J., Meurman, A., Vertex Operator Algebras and the Monster, Academic Press, 1988. MR 90h:17026

[GL] Garland, H., Lepowsky, J., Lie algebra homology and the Macdonald-Kac formulas, Invent. Math. 34 (1976), 37-76. MR 54:2744

[G] Gebert, T., Introduction to vertex algebras, Borcherds algebras and the monster Lie algebra, Inter. J. Mod. Phys. A8 (1993), 5441-5503. MR 95a:17037

[GT] Gebert, T., Teschner, J., On the fundamental representation of Borcherds algebras with one simple imaginary root, Lett. Math. Phys. 31 (1994), 327-334. MR 95h:17027

[GLS] Gorenstein, D., Lyons, R., Solomon, R., The Classification of the Finite Simple Groups, Mathematical Surveys and Monographs 40.1, Amer. Math. Soc., 1994. MR 95m:20014

[HMY] Harada, K., Miyamoto, M., Yamada, H., A generalization of Kac-Moody Lie algebras, J. Algebra 180 (1996), 631-651.

[HJK] Hong, J., Jeong, K.-H., Kwon, J.-H., Integral points on hyperbolas, J. Korean Math. Soc. 34 (1997), 149-157. MR 98e: 11030

[Hu] Hua, L.-K., Introduction to Number Theory, Springer-Verlag, 1982. MR 83f:10001

[J] Jacobson, N., Lie Algebras, 2nd ed., Dover, New York, 1979. MR 80k:17001

[Ju1] Jurisich, E., Generalized Kac-Moody Lie algebras, free Lie algebras, and the structure of the monster Lie algebra, J. Pure and Applied Algebra 126 (1998), 233-266. CMP 98:07

[Ju2] Jurisich, E., An exposition of generalized Kac-Moody algebras, in Lie Algebras and Their Representations, S.-J. Kang, M.-H. Kim, I.-S. Lee (eds), Contemp. Math. 194 (1996), 121-159. MR 97e: 17035

[JW] Jurisich, E., Wilson, R. L., A generalization of Lazard's theorem, preprint.

[JLW] Jurisich, E., Lepowsky, J., Wilson, R. L., Realizations of the Monster Lie algebra, Selecta Mathematica, New Series 1 (1995), 129-161. MR 96e:17059

[K1] Kac, V. G., Simple irreducible graded Lie algebras of finite growth, Math. USSR-Izvestija 2 (1968), 1271-1311. MR 41:4590

[K2] Kac, V. G., Infinite-dimensional Lie algebras and Dedekind's $\eta$-function, Funct. Anal. Appl. 8 (1974), 68-70. MR 51:10410 
[K3] Kac, V. G., Infinite Dimensional Lie Algebras, 3rd ed., Cambridge University Press, 1990. MR 92k:17038

[KK] Kac, V. G., Kang, S.-J., Trace formula for graded Lie algebras and Monstrous Moonshine, in Representatins of Groups, B. Allison, G. Cliff (eds), Canad. Math. Soc. Conf. Proc. 16 (1995), 141-154. MR 96k:17044

[KMW] Kac, V. G., Moody, R. V., Wakimoto, M., On $E_{10}$, in Differential Geometrical Methods in Theoretical Physics, Bleuler, K., Werner, M. (eds), Kluwer Academic Publishers (1988), 109-128. MR 90e:17031

[Ka1] Kang, S.-J., Gradations and structure of Kac-Moody Lie algebras, Yale University Ph. D. dissertation (1990).

[Ka2] Kang, S.-J., Kac-Moody Lie algebras, Spectral sequences, and the Witt formula, Trans. Amer. Math. Soc. 339 (1993), 463-495. MR 93m:17013

[Ka3] Kang, S.-J., Root multiplicities of the hyperbolic Kac-Moody Lie algebra $H A_{1}^{(1)}$, J. Algebra 160 (1993), 492-593. MR 94i:17031

[Ka4] Kang, S.-J., On the hyperbolic Kac-Moody Lie algebra $H A_{1}^{(1)}$, Trans. Amer. Math. Soc. 341 (1994), 623-638. MR 94d:17035

[Ka5] Kang, S.-J., Root multiplicities of Kac-Moody algebras, Duke Math. J. 74 (1994), 635666. MR 95c: 17036

[Ka6] Kang, S.-J., Generalized Kac-Moody algebras and the modular function j, Math. Ann. 298 (1994), 373-384. MR 94m:17026

[Ka7] Kang, S.-J., Root multiplicities of graded Lie algebras, in Lie Algebras and Their Representations, S.-J. Kang, M.-H. Kim, I.-S. Lee (eds), Contemp. Math. 194 (1996), 161-176. MR 97e: 17041

[Ka8] Kang, S.-J., Graded Lie superalgebras and the superdimension formula, J. Algebra 204 (1998), 597-655. CMP 98:13

[KaK1] Kang, S.-J., Kim, M.-H., Free Lie algebras, generalized Witt formula, and the denominator identity, J. Algebra 183 (1996), 560-594. MR 97e:17042

[KaK2] Kang, S.-J., Kim, M.-H., Borcherds superalgebras and a Monstrous Lie superalgebra, Math. Ann. 307 (1997), 677-694. CMP 97:16

[KMN] Kang, S.-J., Kashiwara, M., Misra, K. C., Miwa, T., Nakashima, T., Nakayashiki, A., Affine crystals and vertex models, Inter. J. Mod. Phys. A7 Suppl. 1A (1992), 449-484. MR 94a: 17008

[KM1] Kang, S.-J., Melville, D. J., Root multiplicities of the Kac-Moody algebras $H A_{n}^{(1)}$, J. Algebra 170 (1994), 277-299. MR 95m:17018

[KM2] Kang, S.-J., Melville, D. J., Rank 2 symmetric hyperbolic Kac-Moody algebras, Nagoya Math. J. 140 (1995), 41-75. MR 97c:17039

[Ko] Kostant, B., Lie algebra cohomology and the generalized Borel-Weil theorem, Ann. Math. 74 (1961), 329-387. MR 26:265

[LM] Lepowsky, J., Moody, R. V., Hyperbolic Lie algebras and quasi-regular cusps on Hilbert modular surfaces, Math. Ann. 245 (1979), 63-88. MR 81c:10030

[LZ] Lian, B. H., Zuckerman, G. J., Moonshine cohomology, in Finite Groups and Vertex Operator Algebras, RIMS publication (1995), 87-116. MR 96m:17051

[Li] Liu, L.-S., Kostant's formula for Kac-Moody Lie algebras, J. Algebra 149 (1992), 155178. MR 93d:17011

[M] Macdonald, I. G., Affine root systems and Dedekind's $\eta$-function, Invent. Math. 15 (1972), 91-143. MR 50:9996

[Mo1] Moody, R. V., A new class of Lie algebras, J. Algebra 10 (1968), 211-230. MR 37:5261

[Mo2] Moody, R. V., Root systems of hyperbolic type, Adv. Math. 33 (1979), 144-160. MR 81g:17006

[N] Naito, S., The strong Bernstein-Gelfand-Gelfand resolution for generalized Kac-Moody algebras I: The existence of the resolution, Publ. RIMS, Kyoto Univ. 29 (1993), 709-730. MR 94k: 17040

[P] Peterson, D. H., Freudenthal-type formulas for root and weight multiplicities, preprint (1983).

[S1] Serre, J. P., Lie Algebras and Lie Groups, Benjamin, New York, 1965. MR 36:1582

[S2] Serre, J. P., A Course in Arithmetic, Springer-Verlag, 1973. MR 49:8956

[SUM1] Sthanumoorthy, N., Uma Maheswari, A., Purely imaginary roots of Kac-Moody algebras, Commun. Algebra 24 (1996), 677-693. MR 97c:17041 
[SUM2] Sthanumoorthy, N., Uma Maheswari, A., Root multiplicities of extended hyperbolic KacMoody algebras, Commun. Algebra 24 (1996), 4495-4512. MR 97j:17028

[T] Thompson, J. G., Some numerology between the Fischer-Griess Monster and the elliptic modular function, Bull. Lond. Math. Soc. 11 (1979), 352-353. MR 81j:20030

Department of Mathematics, Seoul National University, Seoul 151-742, Korea

E-mail address: sjkang@math.snu.ac.kr, mhkim@math.snu.ac.kr 